\title{
Assembly of thiosubstituted benzoxazoles via copper-catalyzed coupling of thiols with 5-iodotriazoles serving as diazo surrogates
}

\author{
Yury N. Kotovshchikov, ${ }^{a} *$ Gennadij V. Latyshev, ${ }^{\text {a }}$ Elena A. Kirillova, ${ }^{a}$ \\ Uliana D. Moskalenko, ${ }^{a, b}$ Nikolay V. Lukashev, ${ }^{a}$ and Irina P. Beletskaya ${ }^{a}$ \\ ${ }^{\text {a }}$ Department of Chemistry, M. V. Lomonosov Moscow State University, \\ Leninskiye Gory 1, Moscow, 119991, Russia \\ ${ }^{\mathrm{b}}$ Department of Chemistry, Korea Advanced Institute of Science and Technology, \\ Daejeon, 34141, Republic of Korea \\ E-mail: kotovshchikov@org.chem.msu.ru
}

\section{Table of Contents}

Details of DFT calculations, optimized coordinates and energies of the intermediates S2

Copies of NMR spectra

References 


\section{Details of DFT calculations, optimized coordinates and energies of the intermediates}

The calculations were performed using ORCA 4.2.0 program package. ${ }^{1}$ DFT calculations were performed at $\mathrm{B} 3 \mathrm{LYP}^{2,3} / \mathrm{ma}^{-\mathrm{SVP}^{4,5}}$ level of theory employing def2-ECP pseudopotential for iodine. ${ }^{6}$ Single point energies were evaluated at the same level of theory within the framework of $\mathrm{SMD}$ model $^{7}$ using dioxane as solvent. RIJCOSX ${ }^{8}$ approximation was used to speed up the calculations. Thermodynamic properties were calculated for ideal gas at $298.15 \mathrm{~K}$ using QRRHO approach $^{9}$ for vibrational entropy correction. The nature of optimized intermediates and transition states was verified by frequency analysis. IRC calculations were performed to verify the connectivity of the PES. CYLView ${ }^{10}$ was used to visualize structure of the intermediates.
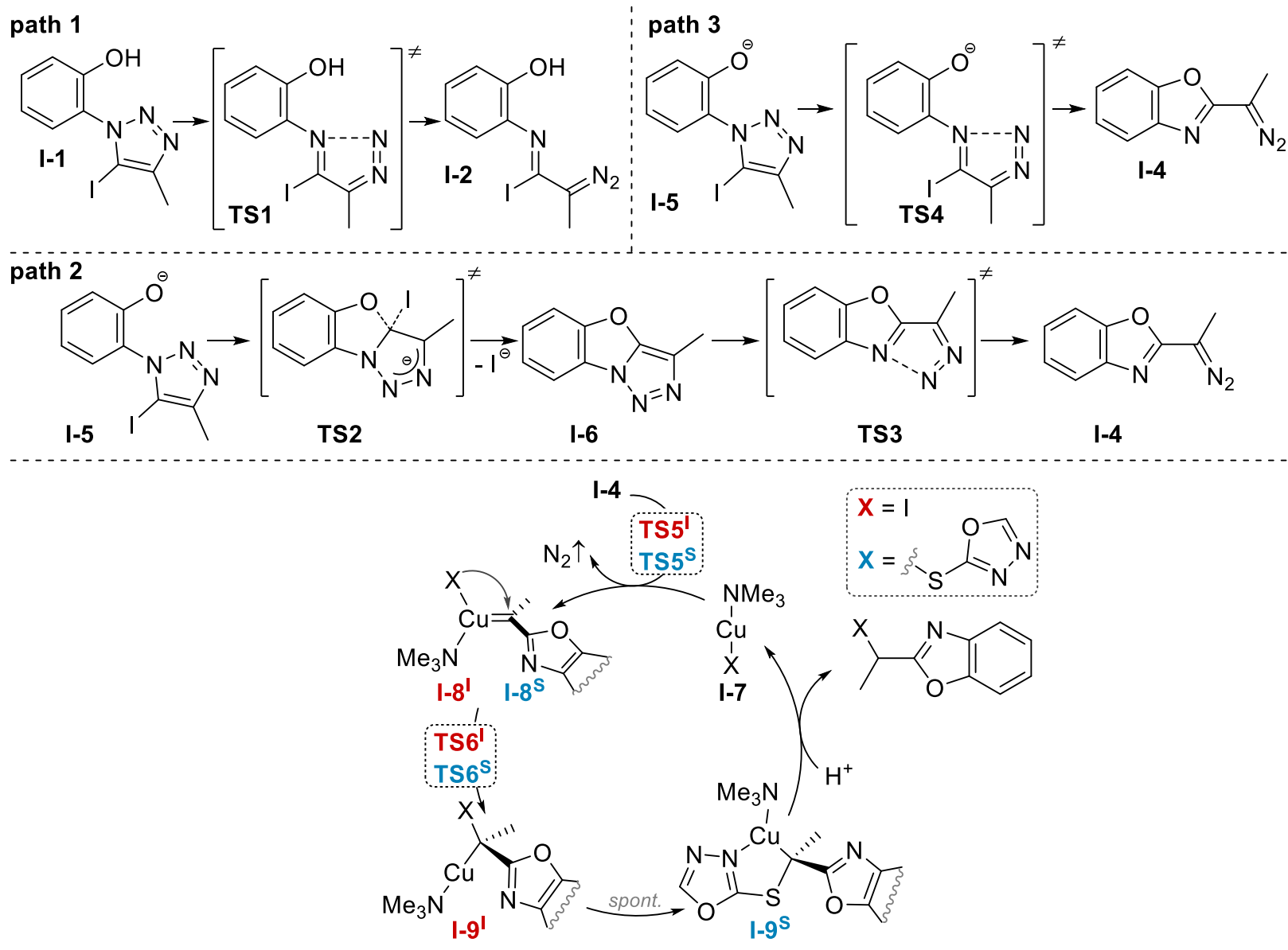

Scheme S1. Naming conventions for DFT calculations. 
I-1

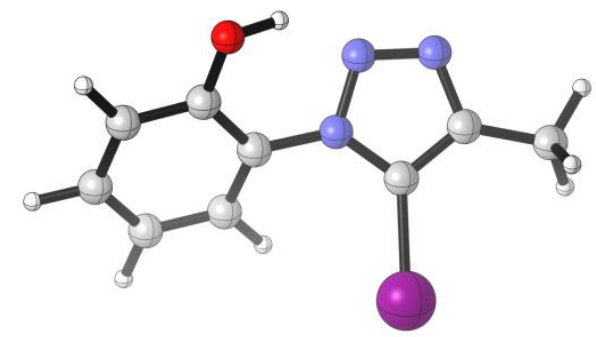

$E_{S M D}=-884.1735 E_{h}$

$\mathrm{ZPE}=0.1579 \mathrm{E}_{\mathrm{h}}$

$\mathrm{G}_{298}=-884.0516 \mathrm{E}_{\mathrm{h}}$

\begin{tabular}{lr} 
C & -2.78196744478608 \\
C & -1.39372534423394 \\
C & -0.71291873282931 \\
C & -1.40330789275122 \\
C & -2.77975338846022 \\
C & -3.46787443298649 \\
H & -3.29714724457504 \\
H & -0.85550847673431 \\
H & -3.30926576102196 \\
H & -4.54730113877390 \\
$\mathrm{~N}$ & 0.68716710347200 \\
$\mathrm{~N}$ & 1.46650909105282 \\
$\mathrm{~N}$ & 2.68489616876032 \\
$\mathrm{C}$ & 2.74749063646878 \\
$\mathrm{C}$ & 1.46117810901953 \\
$\mathrm{I}$ & 0.82653948987228 \\
$\mathrm{C}$ & 4.01577292685093 \\
$\mathrm{H}$ & 4.01230746818577 \\
$\mathrm{H}$ & 4.86262549460193 \\
$\mathrm{H}$ & 4.17479012784255 \\
$\mathrm{O}$ & -0.77596973682034 \\
$\mathrm{H}$ & 0.18666297784595 \\
& \\
\hline
\end{tabular}

0.39380750686894

0.54622016551339

$-0.31643016115331$

$-1.34907715533411$

$-1.49399469710342$

$-0.60767845762236$

1.07422629929584

$-2.04269203615894$

$-2.29912440858038$

$-0.71077662968112$

$-0.12003982262079$

0.22977400296444

0.39398274391498

0.15640032760533

$-0.16336835652742$

$-0.46881070274047$

0.27856286437095

1.17763074450543

0.35693641785466

$-0.59275459176536$

1.50838774719979

1. 31471819919391
$-1.21754007594208$

$-1.06980380341093$

$-0.17414915741350$

0.47475332053771

0.30560501195791

$-0.53347875449836$

$-1.89867902503998$

1.11315531636752

0.81926425954716

$-0.67153823733574$

0.04056337889852

$-1.01141030170244$

$-0.59367915654312$

0.74198507775231

1. 16857852047744

3.13291432108904

1.52128127314668

2.16058402363360

0.82562772315922

2.17594956862428

$-1.78056749662476$

$-1.80551578668052$

\section{TS1}

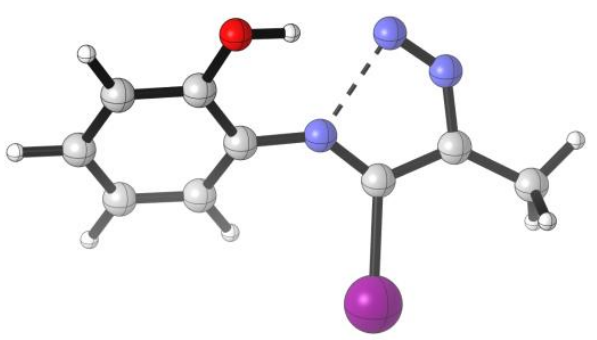

$\begin{array}{ll}\text { C } & -2.82304791679492 \\ \text { C } & -1.44500518540432 \\ \text { C } & -0.75176677222183 \\ \text { C } & -1.45437100116446 \\ \text { C } & -2.82304299221889 \\ \text { C } & -3.50742735987520 \\ \text { H } & -3.33502767006479 \\ \text { H } & -0.91442340763099 \\ \text { H } & -3.35379131596437 \\ \text { H } & -4.58088071113534 \\ \text { N } & 0.61057952667120 \\ \text { N } & 2.06402901300557 \\ \text { N } & 2.90425640761264 \\ \text { C } & 2.85367066487829 \\ \text { C } & 1.46915950231096 \\ \text { I } & 1.01971372680409\end{array}$
$E_{S M D}=-884.1326 E_{h}$

$\mathrm{ZPE}=0.1577 \mathrm{E}_{\mathrm{h}}$

$\mathrm{G}_{298}=-884.0150 \mathrm{E}_{\mathrm{h}}$

Imaginary frequency $=217.33 i \mathrm{~cm}^{-1}$
0.33497839330228
0.50970192031194
$-0.29920233042555$
$-1.31247539640453$
$-1.48737028293305$
$-0.65387468235028$
0.97924002942428
$-1.96818725223557$
$-2.27720776333032$
$-0.78492674606741$
$-0.06174242253598$
0.46351305769673
0.46098895794243
0.18716320425247
$-0.09112890331099$
$-0.42513357665717$

$-1.25880913586520$

$-1.07826680519613$

$-0.13649406833052$

0.54495364731343

0.34398409425788

$-0.55139548591939$

$-1.97668023484798$

1.22866196704855

0.88063703656683

$-0.71091401100998$

0.00536275174991

$-1.48512486827839$

$-0.66315159197281$

0.64548234603154

0.95557984369223

3. 03181330991702 


\section{I-2}

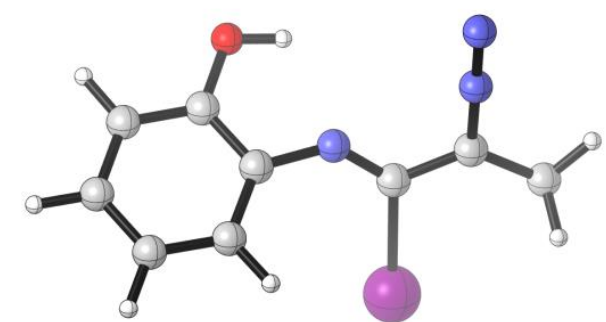

$$
\begin{aligned}
& E_{S M D}=-884.1493 E_{h} \\
& Z P E=0.1579 E_{h} \\
& G_{298}=-884.0334 E_{h}
\end{aligned}
$$

C

$-2.72115352723905$

C $\quad-1.34698079914536$

C $\quad-0.82919203184858$

C $\quad-1.72136393173975$

C $\quad-3.09344629762659$

C $\quad-3.58884061086118$

H $\quad-3.09090239756811$

$\mathrm{H} \quad-1.33312654845385$

H $\quad-3.77692623315676$

$\mathrm{H} \quad-4.66292854444629$

N $\quad 0.54395367306019$

$\mathrm{N} \quad 3.13043241912114$

N $\quad 2.98232696971208$

C $\quad 2.79660116959697$

C $\quad 1.43899223649425$

I $\quad 1.10428325106602$

C $\quad 3.99273710632393$

H $\quad 3.84386884782810$

H $\quad 4.88722434806899$

$\mathrm{H} \quad 4.19039837094557$

$0 \quad-0.51303261380198$

H $\quad 0.38166514367027$
$-1.15786235951820$ $-0.94601246291178$ $-0.42689626828376$ $-0.08609480291242$ $-0.28855912726745$ $-0.83299512889918$ $-1.56774587646306$ 0.35801554867142 $-0.01383025981416$ $-0.99444387610517$ $-0.20656021722029$

1.57675598385450

0.92536529729262

0.17552160134549 $-0.31954972897202$ $-1.31098698237603$ $-0.08068395232351$ 0.33668782699069 0.38961350883423 $-1.15897617536591$ $-1.24507536239039$ $-0.96642418616565$
$-1.46224620601898$

$-1.31575152051869$

$-0.10047315662279$

0.93311955405250

0.77605309437283

$-0.41671493525212$

$-2.40445448648347$

1.85033090308055

1.58268503402692

$-0.53997334027182$

$-0.07608240975332$

$-1.33309734525484$

$-0.41434644726190$

0.65121216671509

0.80622493428014

2.77880434215351

1.53790068689564

2. 54628556066427

1.10413893401186

1.64150745273847

$-2.33871640715956$

$-2.07895340839427$

\section{I-4}

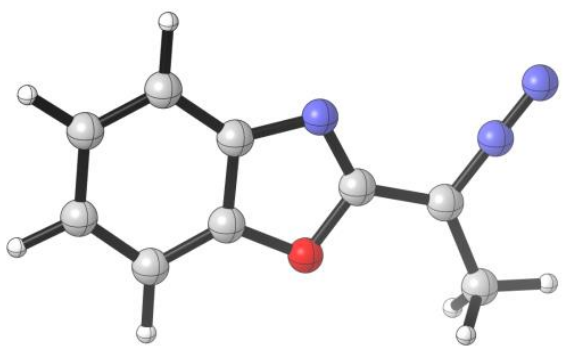

$$
\begin{aligned}
& E_{S M D}=-585.8615 E_{h} \\
& Z P E=0.1476 E_{h} \\
& G_{298}=-585.7500 E_{h}
\end{aligned}
$$

$\begin{array}{lr}\mathrm{C} & -0.74363797134850 \\ \mathrm{C} & -0.95496397161622 \\ \mathrm{O} & 0.24862863498342 \\ \mathrm{~N} & 0.59278060494914 \\ \mathrm{C} & -1.81587995014825 \\ \mathrm{H} & -1.67368484043721 \\ \mathrm{C} & -2.18196414505336\end{array}$

1.02903156423460

$-0.35975843349473$

$-0.93552284853582$

1.29373094049124

1.87407288693263

2.95431079503839

$-0.97313359409649$
0.11995211125899

0.03618639346741

$-0.28808559122578$

$-0.15363938523849$

0.43567436376908

0.50505571354913

0.24815652257619 


$\begin{array}{lr}\mathrm{H} & -2.30974512195507 \\ \mathrm{C} & -3.24571479196047 \\ \mathrm{H} & -4.23746529635264 \\ \mathrm{C} & -3.06293053256101 \\ \mathrm{H} & -3.92033961424291 \\ \mathrm{C} & 1.11784348373532 \\ \mathrm{C} & 2.47900375992853 \\ \mathrm{~N} & 4.06110096717495 \\ \mathrm{~N} & 3.32111879612898 \\ \mathrm{C} & 2.95960935612869 \\ \mathrm{H} & 2.42624170247813 \\ \mathrm{H} & 2.78958940241982 \\ \mathrm{H} & 4.03511052774867\end{array}$
$-2.05459809753085$
$-0.11517089548007$
$-0.53821174615252$
1. 27929547245901
1.91011979350790
0.12428020876904
$-0.20981637791313$
1.64803880149486
0.78717776312213
$-1.62032529758504$
$-2.07760479728445$
$-2.25614802373380$
$-1.62657011424287$

0.17338384560031

0.56198789061013

0.73980288855424

0.65398238846329

0.90195270781809

$-0.38284090344059$

$-0.72619834617269$

$-0.90274878999953$

$-0.81828030954908$

$-0.97343747370159$

$-1.82287285035433$

$-0.08955907358775$

$-1.19937310239705$

I-5

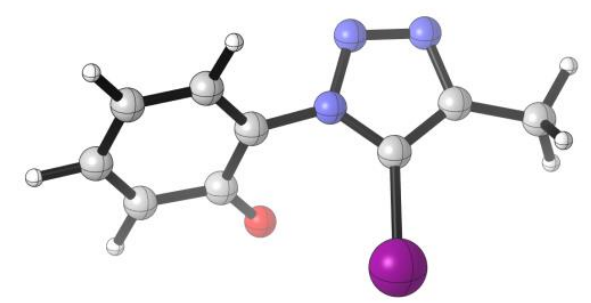

$$
\begin{aligned}
& E_{S M D}=-883.6664 E_{h} \\
& Z P E=0.1469 E_{h} \\
& G_{298}=-883.5590 E_{h}
\end{aligned}
$$

$\begin{array}{lr}\text { C } & -2.43741799514555 \\ \text { C } & -1.02498225739179 \\ \text { C } & -0.77318127547072 \\ \text { C } & -1.78756959168338 \\ \text { C } & -3.13187489050119 \\ \text { C } & -3.43410422293577 \\ \text { H } & -2.68668539848717 \\ \text { H } & -1.50525042164516 \\ \text { H } & -3.92213331930162 \\ \text { H } & -4.48287061109779 \\ \text { N } & 0.58761335287557 \\ \text { N } & 1.08841411902619 \\ \text { N } & 2.34730490803906 \\ \text { C } & 2.70240112239454 \\ \text { C } & 1.57098343974327 \\ \text { I } & 1.32277714255302 \\ \text { C } & 4.08561495694819 \\ \text { H } & 4.09768461313058 \\ \text { H } & 4.71021395236146 \\ \text { H } & 4.54687812453887 \\ \text { O } & -0.10781774795053\end{array}$

$-1.50417034594732$

$-1.26927889045215$

$-0.11743507720552$

0.69747000370606

0.42700855039512

$-0.69221970925172$

$-2.36198793761730$

1. 55776651170602

1.06445137987912

$-0.92460842206395$

0.23415264404335

1. 43518502540337

1.47648536261761

0.29917849228610

$-0.50682771930466$

$-2.38804799469901$

0.03139979314717

$-0.19332014362917$

0.91930798312404

$-0.82766074819811$

$-1.98363675793906$
$-1.13772944777161$

$-0.90455879014905$

$-0.04916928661888$

0.45969877292035

0.18861322825974

$-0.61719540846844$

$-1.77045432562161$

1.07425322228088

0.59345879936325

$-0.84063546997045$

0.22230533271866

$-0.13276898123870$

0.21800060083514

0.79645370063090

0.80422005894828

1.66640384196980

1. 29553280247448

2. 37609878430887

1.11774375284700

0.77854833712975

$-1.39141852484833$

\section{TS2}

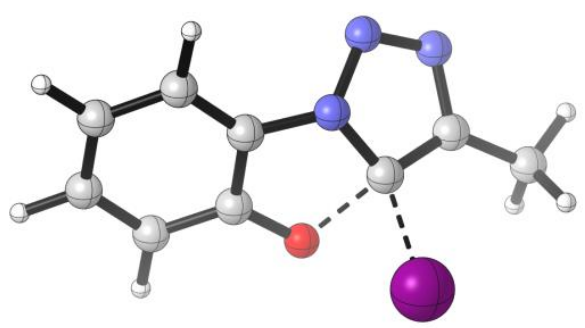

$$
\begin{aligned}
& E_{S M D}=-883.6266 E_{h} \\
& Z P E=0.1462 E_{h} \\
& G_{298}=-883.5191 E_{h}
\end{aligned}
$$

Imaginary frequency $=364.09 i \mathrm{~cm}^{-1}$

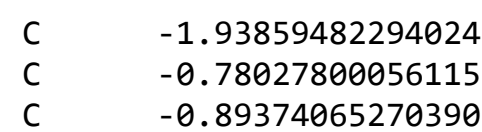

-1.51590354861244
-1.03448059246969
0.18776113429945

$-0.98453416136724$

$-0.33713180514052$

0.39293739579460 


$\begin{array}{ll}\text { C } & -2.08097899867166 \\ \mathrm{C} & -3.22571697022456 \\ \mathrm{C} & -3.14092193430342 \\ \mathrm{H} & -1.88070950119632 \\ \mathrm{H} & -2.10750940759455 \\ \mathrm{H} & -4.17431186616671 \\ \mathrm{H} & -4.03575501665938 \\ \mathrm{~N} & 0.38130030789862 \\ \mathrm{~N} & 0.98645089328006 \\ \mathrm{~N} & 2.25639481499540 \\ \mathrm{C} & 2.53691947103592 \\ \mathrm{C} & 1.32144506756391 \\ \mathrm{I} & 1.31412999001228 \\ \mathrm{C} & 3.92841453997742 \\ \mathrm{H} & 4.19247357386174 \\ \mathrm{H} & 4.63385875445100 \\ \mathrm{H} & 4.07204716068651 \\ \mathrm{O} & 0.40108059725903\end{array}$
0.89613339372557
0.39161383336693
$-0.80264654341244$
$-2.44322888213249$
1.83110791062935
0.93099350785181
$-1.19341800823444$
0.54098684905801
1.77211251528821
1.53761069027139
0.20733829118142
$-0.45749783986079$
$-2.14445677414836$
$-0.33016134597052$
$-0.87028437303443$
0.50465784294808
$-1.03785052089499$
$-1.59717553984960$

0.49776846784098

$-0.14528744831259$

$-0.87492421907350$

$-1.55953733185311$

1.06206528322632

$-0.07620482944810$

$-1.37081937307107$

0.87476179457345

0.63585034178002

0.53539379979295

0.66244827989209

0.87410811038591

2.49841550596329

0.60939108568443

1.53667329419414

0.47797691687079

$-0.22696720111620$

$-0.33498290661660$

$$
\begin{aligned}
& E_{S M D}=-297.8441 E_{h} \\
& G_{298}=-297.8610 E_{h}
\end{aligned}
$$

0.0

0.0

\section{I-6}

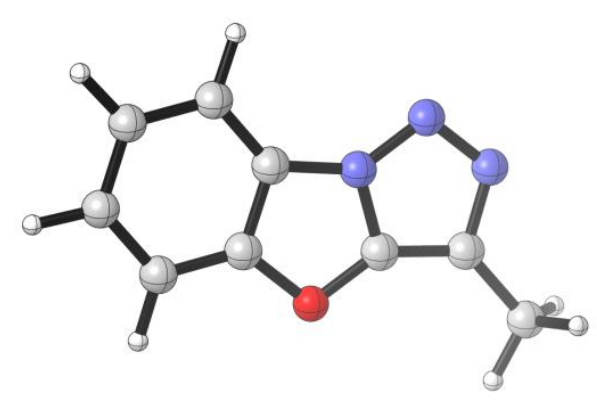

$\begin{array}{lr}\text { C } & -0.59971946283806 \\ \text { C } & -1.03102513751878 \\ \text { O } & 0.02815908196658 \\ \text { N } & 0.76949569353251 \\ \text { C } & -1.48405535785313 \\ \text { H } & -1.14371762032616 \\ \text { C } & -2.35328028934227 \\ \text { H } & -2.67316795669865 \\ \text { C } & -3.24735293388974 \\ \text { H } & -4.29923871116383 \\ \text { C } & -2.82238716128262 \\ \text { H } & -3.55060948569235 \\ \text { C } & 1.11176191846166 \\ \text { C } & 2.46852590436313 \\ \text { N } & 1.84194512797994 \\ \text { N } & 2.84528786287930 \\ \text { C } & 3.42663312356045 \\ \text { H } & 3.91157461057822 \\ \text { H } & 2.90995062664905 \\ \text { H } & 4.22327516663474\end{array}$
0.88463497221779 $-0.45113197757373$

$-1.27819335400625$

0.82526467109556

1.90374039819570

2.93476152370563

$-0.82111075264365$

$-1.85853551468950$

0.20494582726600

$-0.04103086235045$

1. 53724280099267

2. 30593388075735

$-0.46270940284710$

$-0.44784892934491$

1.63938363398933

0.86586888646047

$-1.53683981576453$

$-1.33309590527559$

$-2.50507652026006$ $-1.62663855992474$
0.10483800410907

$-0.03104571813845$

$-0.36908950955182$

$-0.16768838231628$

0.44444540204776

0.54915917470802

0.16261031262919

0.05320946464174

0.50464373369707

0.66715040853044

0.64290012349198

0.91136441184174

$-0.44274244594388$

$-0.70108196361603$

$-0.24248422575749$

$-0.56052615142867$

$-1.05815708355673$

$-2.02627336804206$

$-1.13013934592330$

$-0.30219784142229$ 


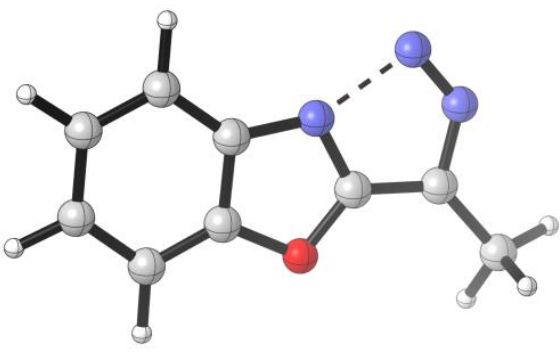

$$
\begin{aligned}
& E_{S M D}=-585.8275 E_{h} \\
& Z P E=0.1471 E_{h} \\
& G_{298}=-585.7161 E_{h}
\end{aligned}
$$

Imaginary frequency $=258.38 i \mathrm{~cm}^{-1}$

$\begin{array}{lr}\text { C } & -0.58123900480581 \\ \text { C } & -0.72412133358486 \\ \text { O } & 0.51899973075700 \\ \text { N } & 0.76428920670479 \\ \text { C } & -1.69466100029791 \\ \text { H } & -1.60226391259694 \\ \text { C } & -1.93213211251754 \\ \text { H } & -2.00868926479275 \\ \text { C } & -3.04522633185817 \\ \text { H } & -4.02425884497373 \\ \text { C } & -2.92516848779595 \\ \text { H } & -3.81630072127497 \\ \text { C } & 1.36008834322416 \\ \text { C } & 2.74433913700602 \\ \text { N } & 2.30607545445574 \\ \text { N } & 3.06631959306481 \\ \text { C } & 3.71208254221917 \\ \text { H } & 3.22558067984224 \\ \text { H } & 4.12456507018297 \\ \text { H } & 4.55583025704172\end{array}$

0.56930868340419

$-0.80916391227095$

$-1.32252634614969$

0.86811627336659

1. 33032843830077

2. 39787779309149

$-1.48291625306535$

$-2.55107940492715$

$-0.70916929388317$

$-1.18766831917571$

0.66619140131504

1.23204156283351

$-0.26601773397912$

$-0.19937762718254$

1.96522161778769

1.09599412972582

$-1.27287769506188$

$-2.02503889460973$

$-1.80172696945228$

$-0.84588045006751$
0.08071045166955

$-0.18109518783914$

$-0.51613676363504$

$-0.10288784471852$

0.44455387269176

0.65110924612789

$-0.10103811587024$

$-0.31149727436852$

0.26408771598388

0.34244047342049

0.53149666918200

0.81364860922840

$-0.45193920475634$

$-0.70890023510012$

$-0.17658095270401$

$-0.50031040347111$

$-1.08764646263032$

$-1.72781698890073$

$-0.20977000496247$

$-1.64923259934745$

TS4

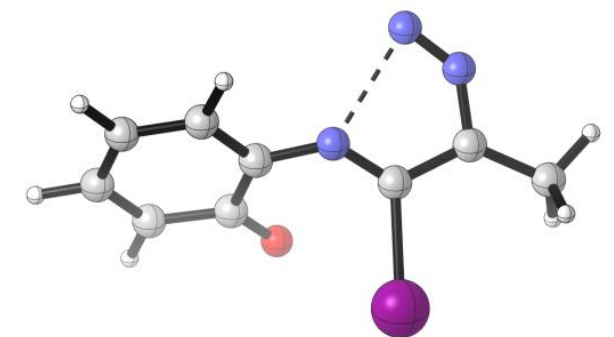

$\mathrm{E}_{\mathrm{SMD}}=-883.6178 \mathrm{E}_{\mathrm{h}}$

$\mathrm{ZPE}=0.1431 \mathrm{E}_{\mathrm{h}}$

$\mathrm{G}_{298}=-883.5145 \mathrm{E}_{\mathrm{h}}$

Imaginary frequency $=170.65 i \mathrm{~cm}^{-1}$

$\begin{array}{ll}\text { C } & -2.44295349523686 \\ \text { C } & -1.05584521216713 \\ \text { C } & -0.86237330049571 \\ \text { C } & -1.94701754324816 \\ \text { C } & -3.26117020837842 \\ \text { C } & -3.49188302675108 \\ \text { H } & -2.62762032850136 \\ \text { H } & -1.72747386222630 \\ \text { H } & -4.09091605553557 \\ \text { H } & -4.51931071224750 \\ \text { N } & 0.41553366099664 \\ \text { N } & 1.65150893459447 \\ \text { N } & 2.56101413613519 \\ \text { C } & 2.75655416489256 \\ \text { C } & 1.47337902155922 \\ \text { I } & 1.50710217104313 \\ \text { C } & 4.10680886452163\end{array}$

$-1.59312621322137$

$-1.31267088703525$

$-0.12092795862004$

0.69254799615743

0.36901104684286

$-0.79300352885345$

$-2.48407361787644$

1.59209931710638

1.00425921423700

$-1.06330559932636$

0.28337213567957

2. 25953053025905

1.64360492857272

0.38179281463610

$-0.30506542709703$

$-2.30511870016204$

$-0.12190710471531$
$-1.09162895788136$

$-0.77732310431029$

0.06545205266650

0.44210503356833

0.10420235309686

$-0.66637255642802$

$-1.70027514018063$

1.02582350133536

0.42655088148724

$-0.93983107288953$

0.39350841123385

$-0.01435839236618$

0.40101009468827

0.78628073564157

0.76397186179311

1.64717354853769

1.19391002725460 


$\begin{array}{rrrr}H & 4.15930878227924 & -0.39829305779419 & 2.26224546776010 \\ H & 4.85329454996275 & 0.66647930220910 & 1.01051665747759 \\ H & 4.40561801804065 & -1.01281490589470 & 0.61568059533575 \\ O & -0.09756055923738 & -2.00917828510403 & -1.20124099782078\end{array}$

I-7 $^{\mathrm{I}}$

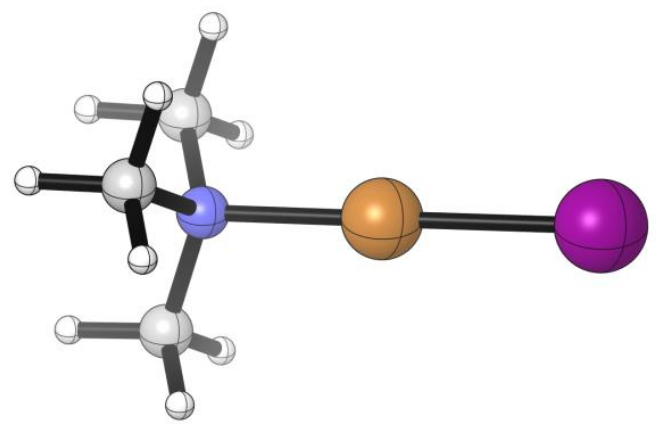

$\mathrm{E}_{\mathrm{SMD}}=-2112.2574 \mathrm{E}_{\mathrm{h}}$

$\mathrm{ZPE}=0.1231 \mathrm{E}_{\mathrm{h}}$

$\mathrm{G}_{298}=-2112.1687 \mathrm{E}_{\mathrm{h}}$

$\begin{array}{lr}\mathrm{N} & 0.00194738178649 \\ \mathrm{Cu} & -0.00314496318818 \\ \mathrm{I} & -0.01785328812491 \\ \mathrm{C} & -1.39740961225133 \\ \mathrm{H} & -1.92114553297033 \\ \mathrm{H} & -1.92108648591383 \\ \mathrm{H} & -1.43555670419057 \\ \mathrm{C} & 0.70091973463858 \\ \mathrm{H} & 0.19683861490610 \\ \mathrm{H} & 1.73894728226291 \\ \mathrm{H} & 0.71038590874408 \\ \mathrm{C} & 0.70093166973999 \\ \mathrm{H} & 0.71032951480365 \\ \mathrm{H} & 1.73897898825574 \\ \mathrm{H} & 0.19691749350159\end{array}$

$-0.00019050472595$

$-0.00013842376328$

0.00123364437206

$-0.00010128217524$

0.89195831337225

$-0.89239694282556$

0.00018807337332

$-1.21081878432934$

$-2.11214925361511$

$-1.21352023512654$

$-1.24400936146693$

1. 21064467841428

1. 24437131335693

1. 21312024628009

2. 11180851885901
2.57699028686962

0.56968076054747

$-1.85529488510510$

3.05599803562043

2.68420862069432

2. 68468724493458

4.16342517295654

3.06111255960190

2.68432880524803

2. 69951610531687

4.16870175257110

3.06058745026125

4. 16815925552129

2.69904386164934

2. 68331818231234

$\mathrm{I}^{-7^{\mathrm{S}}}$

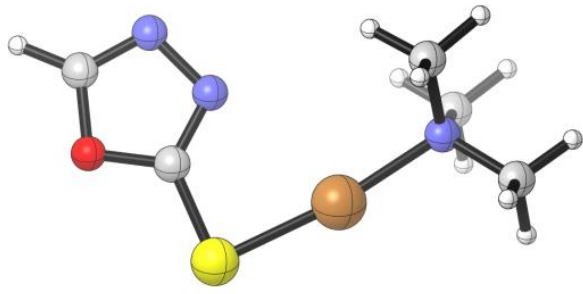

$\begin{array}{lr}\mathrm{N} & -2.44045829388498 \\ \mathrm{Cu} & -0.48714173140402 \\ \mathrm{~S} & 1.71778554124597 \\ \mathrm{C} & 1.82335414339823 \\ \mathrm{~N} & 0.84504736657039 \\ \mathrm{~N} & 1.42626698321167 \\ \mathrm{C} & 2.70057002872581 \\ \mathrm{O} & 3.03425412867033 \\ \mathrm{H} & 3.50036611019343 \\ \mathrm{C} & -3.26813914166373 \\ \mathrm{H} & -2.93774099624241 \\ \mathrm{H} & -3.17023578214755 \\ \mathrm{H} & -4.33617820228037 \\ \mathrm{C} & -2.87182785246910 \\ \mathrm{H} & -2.77437540878993 \\ \mathrm{H} & -2.24132722936780\end{array}$

$E_{S M D}=-2473.7505 E_{h}$

$\mathrm{ZPE}=0.1603 \mathrm{E}_{\mathrm{h}}$

$\mathrm{G}_{298}=-2473.6297 \mathrm{E}_{\mathrm{h}}$
0.55722176500613

0.97452814343016

1.15100009601438

$-0.54855882180491$

$-1.40723563263988$

$-2.64683008168392$

$-2.46376263846393$

$-1.17305186481734$

$-3.19089000518388$

1. 76226445636932

2. 29509738955459

2. 43804577662129

1.49213454474538

$-0.15684059442730$

0.50311928241365

$-1.04381337700960$
$-0.07593828357656$

$-0.26928042648111$

$-0.41964395455407$

$-0.09708233065544$

0.08887067135258

0.31887606177288

0.25559107036823

$-0.00200124002546$

0.38159559801243

0.12903394502800

1.03221626129855

$-0.73288442105864$

0.24844236026903

$-1.29816747648417$

$-2.17246107566405$

$-1.45098947160971$ 


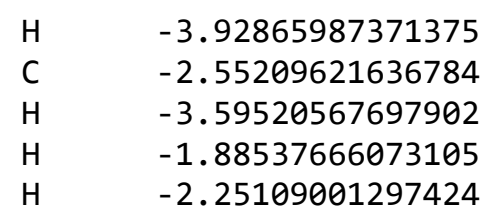

$\mathbf{N}_{2}$

N $\quad-0.55035655001140$

$\mathrm{N} \quad 0.55035655001140$
$-0.47846851432434$

$-0.34651772875762$

$-0.69863949980307$

$-1.20959095352877$

0.18785611228970
$-1.21198962193208$

1.09390364344966

1. 21692712519122

0.95857778716103

2.00640377813770
TS5 $^{\mathrm{I}}$

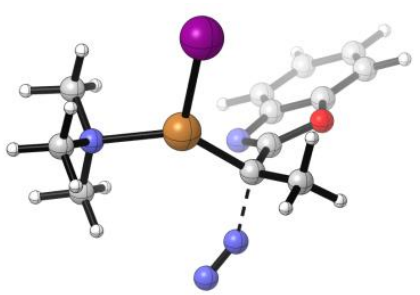

C $\quad 5.46667417379203$

C $\quad 5.85542211601450$

O 4.84432770698412

$\mathrm{N} \quad 4.22912066759911$

C $\quad 6.31566196607913$

$\mathrm{H} \quad 6.03072457640533$

C $\quad 7.06457389912264$

$\mathrm{H} \quad 7.34051017245902$

C $\quad 7.89856917770374$

H $\quad 8.86063800328286$

C $\quad 7.53016573539991$

$\mathrm{H} \quad 8.21933108129203$

C $\quad 3.90772101374323$

C 2.44691857782920

$\mathrm{H} \quad 3.19393394043482$

$\mathrm{H} \quad 2.56841774039757$

$\mathrm{H} \quad 1.45702908347199$

C $\quad 2.69558691652425$

$\mathrm{N} \quad 1.43024856853789$

$\mathrm{N} \quad 0.54339347106504$

N $\quad 1.91816971602673$

Cu 2.68050462232245

I $\quad 4.02803248783495$

C $\quad 1.01930049407467$

$\mathrm{H} \quad 0.16340223867283$

$\mathrm{H} \quad 1.56125377409560$

H $\quad 0.63357282507879$

C $\quad 3.07150124540278$

H $\quad 3.62837242107435$

$\mathrm{H} \quad 3.74537976974732$

H 2.74043390119272

C $\quad 1.19672841949355$

H $\quad 0.80312755936127$

$\mathrm{H} \quad 1.87011528556011$

$\mathrm{H} \quad 0.34695824792271$

$$
\begin{aligned}
& E_{S M D}=-109.3864 E_{h} \\
& Z P E=0.0056 E_{h} \\
& G_{298}=-109.3992 E_{h}
\end{aligned}
$$
0.00000000000000
0.00000000000000
0.00000000000000
0.00000000000000

$$
\begin{aligned}
& E_{S M D}=-2698.0883 E_{h} \\
& Z P E=0.2682 E_{h} \\
& G_{298}=-2697.8701 E_{h}
\end{aligned}
$$

Imaginary frequency $=492.54 i \mathrm{~cm}^{-1}$

\subsection{8}

3.42804523106494

3.41771380607483

2.04678416031545

2.42059932499344

1.77380170058804

4.11424254650347

4.75028891774948

3.93694784321280

4.45422113284296

3.10778357326823

3.00083612600210

2. 55792980434589

3.18022030838565

2. 89707631793985

4. 25486706184965

2. 99449101137762

2. 27671442929251

2.74330840933553

2. 52768321460061

$-1.38549226533156$

0.32506966223035

$-0.42166863386029$

$-2.12247415316556$

$-1.48882395421962$

$-2.39361884870888$

$-3.04379854867773$

$-2.22848283691631$

$-2.50489467748275$

$-1.66857759165419$

$-3.14901288991575$

$-0.95673450408389$

$-1.82577021046129$

$-0.38680635086265$

$-0.31380319783677$
0.69044038245883

$-0.37223936359431$

$-1.28823041258091$

0.38683276485483

1. 79483709675192

2.62682920500006

$-0.41333218555364$

$-1.25592851464892$

0.69552049291209

0.72539519658751

1.77850898698677

2.61989823717083

$-0.76813672620111$

$-2.74739086128937$

$-3.50596586264642$

$-2.53704909742238$

$-3.18813877535050$

$-1.56005975781747$

$-0.43789274821141$

0.20314980185349

$-0.79290565057317$

$-1.82341123584918$

$-3.87133129853801$

$-1.69800358997192$

$-1.97439140895610$

$-2.61487540606297$

$-1.21599804267417$

$-0.42616046255793$

$-1.33218302772882$

0.23822419772242

0.09602799351180

0.41525634512066

0.98150421972972

1.07170001080140

0.14078612876644 
TS5 $^{\text {S }}$

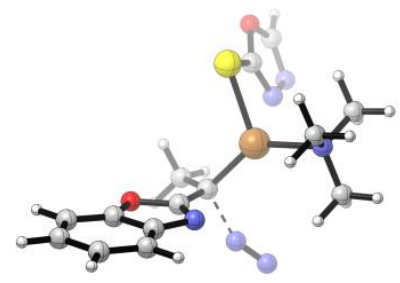

C $\quad-0.47002255877393$

C $\quad-1.44275703884575$

O -1.90677556494410

$\mathrm{N} \quad-0.32930125869771$

C $\quad 0.17457423531494$

$\mathrm{H} \quad 0.93010488804905$

C $\quad-1.81420512391516$

$\mathrm{H} \quad-2.56795123437015$

C $\quad-1.16507856096981$

H $\quad-1.41927480893264$

C $\quad-0.18961728391738$

$\mathrm{H} \quad 0.28971600043685$

C $\quad-1.18666478786158$

C $\quad-2.57309678817834$

$\mathrm{H} \quad-2.24044501060096$

$\mathrm{H} \quad-3.50823216908883$

$\mathrm{H} \quad-2.75366339855032$

C $\quad-1.42361353305763$

$\mathrm{N} \quad 1.93656583069706$

Cu $\quad 0.30032130535840$

S $\quad 1.00579811065445$

C $\quad 0.26274730726297$

$\mathrm{N} \quad-0.32995038859706$

$\mathrm{N} \quad-0.78776213907402$

C $\quad-0.44794623636194$

$0 \quad 0.21463716001280$

$\mathrm{H} \quad-0.62593576918114$

C $\quad 1.48430381860094$

H 2.31291312388357

H $\quad 1.09479213070838$

$\mathrm{H} \quad 0.68468460832895$

C $\quad 2.44545260901592$

$\mathrm{H} \quad 1.65069469173000$

$\mathrm{H} \quad 2.75538343057814$

H $\quad 3.31281906233468$

C $\quad 2.97659279808074$

$\mathrm{H} \quad 3.86780932368026$

$\mathrm{H} \quad 3.28190318757298$

$\mathrm{H} \quad 2.57943344042957$

N $\quad-2.00453225138721$

N $\quad-1.95177588442504$
$\mathrm{E}_{\mathrm{SMD}}=-3059.5797 \mathrm{E}_{\mathrm{h}}$

$\mathrm{ZPE}=0.3055 \mathrm{E}_{\mathrm{h}}$

$\mathrm{G}_{298}=-3059.3274 \mathrm{E}_{\mathrm{h}}$

Imaginary frequency $=493.48 i \mathrm{~cm}^{-1}$
$-6.27223867569682$

$-6.43154527799205$

$-5.18917295279585$

$-4.91916725497853$

$-7.39730463480654$

$-7.29046522898315$

$-7.66522628845557$

$-7.75786853710897$

$-8.77700249360827$

$-9.77465496491021$

$-8.64503588437572$

$-9.54522862366887$

$-4.32843606206791$

$-2.46300019907854$

$-2.66306407446101$

$-3.02237284044271$

$-1.38191132899476$

$-2.87951969086856$

$-1.35952709522919$

$-1.97489898038941$

$-1.88397987840860$

$-0.33325636735966$

0.24730859813708

1. 48465342867975

1.57850263604963

0.48282846135067

2. 39923325344444

$-1.27325690834735$

$-0.98023224476463$

$-2.24755052252149$

$-0.52317687423916$

$-0.05494729222826$

0.70032429512192

$-0.13138777580607$

0.27016842231689

$-2.39351455169727$

$-2.15208707689033$

$-2.46710910780857$

$-3.36784617736838$

$-2.47076891621868$

$-1.94645636352783$
$-0.93039689492349$

0.07553047382873

0.39186727024531

$-1.19033839425158$

$-1.46708585283892$

$-2.24799840168152$

0.60075590201362

1. 38452386788178

0.05426280669375

0.42077437733672

$-0.95994452922687$

$-1.35243415662567$

$-0.40625834653601$

0.61204874569123

1.64390861370792

0.44885735842539

0.53942004779876

$-0.27567977943054$

$-1.24125798464728$

$-0.00142313432624$

2. 27917490186677

2. 25269744660258

1. 22942036009353

1.66037137299282

2. 90076417005642

3. 35213850688609

3. 59295258316361

$-2.63744644109840$

$-3.31502640331165$

$-2.96688506820301$

$-2.72773909736888$

$-0.78379144936487$

$-0.85324552770351$

0.26760748127507

$-1.39401446632097$

$-1.10101548959951$

$-1.71615064243993$

$-0.04757144501941$

$-1.42127816055209$

$-1.88550970285993$

$-2.86727337622963$ 
I-8

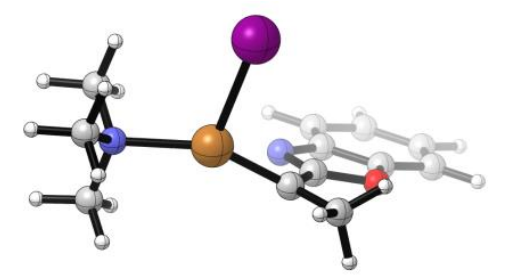

C $\quad-1.54583763934521$

O $\quad-2.16489503647284$

$\mathrm{N} \quad 0.01039460716347$

C $\quad 0.71572900101285$

$\mathrm{H} \quad 1.77391576016587$

C $\quad-2.08335613345511$

H $\quad-3.14227699667786$

C $\quad-1.17927569446301$

H $\quad-1.54147342218588$

C $\quad 0.19469657065932$

H $\quad 0.85661029864342$

C $\quad-1.16603070078756$

C $\quad-2.90786035893378$

H $\quad-3.37949863480976$

H $\quad-3.46905679804090$

H $\quad-3.05251341708023$

C $\quad-1.47490646197702$

$\mathrm{N} \quad 1.66962876164510$

$\mathrm{Cu} \quad 0.01396625489683$

I $\quad-0.06234630152770$

C $\quad 2.87511846655702$

H $\quad 2.81938143085285$

H $\quad 2.92964756549611$

H $\quad 3.79229475263569$

C $\quad 1.57125844861471$

H $\quad 0.66795298643897$

H $\quad 1.50061606739769$

H $\quad 2.45481674829606$

C $\quad 1.71699572389998$

H $\quad 0.81138810574495$

H $\quad 2.60305129495108$

H $\quad 1.76420198254511$
$\mathrm{E}_{\mathrm{SMD}}=-2588.7348 \mathrm{E}_{\mathrm{h}}$

$\mathrm{ZPE}=0.2608 \mathrm{E}_{\mathrm{h}}$

$\mathrm{G}_{298}=-2588.5215 \mathrm{E}_{\mathrm{h}}$
$-6.53927070039664$

$-6.77544817475098$

$-5.57554405974808$

$-5.18786793728044$

$-7.62135709668337$

$-7.45203900312394$

$-8.04933409047713$

$-8.20535928951885$

$-9.10942276148519$

$-10.13185532571649$

$-8.89957510494297$

$-9.76647827665764$

$-4.63310109967303$

$-2.87010355351120$

$-3.41128349941908$

$-3.17264086345260$

$-1.79054983289346$

$-3.23181015510250$

$-1.08456578998057$

$-2.11539348019714$

$-1.82667147058411$

$-1.67766159101689$

$-1.57502873789091$

$-2.74787295251839$

$-1.17722500142069$

0.34442421175982

0.77976727726118

0.44857729610561

0.90416055858392

$-1.24700446883179$

$-0.81956141755193$

$-0.73767622103119$

$-2.31513481285327$
$-0.27957536624766$

$-0.00204300356465$

0.04760095032008

$-0.41103679675799$

$-0.38230662640880$

$-0.59053682028320$

0.17262430950813

0.38524103539908

0.06847637121240

0.20333002676245

$-0.20363648807690$

$-0.27207364789615$

$-0.19870364429043$

$-0.00617109894615$

0.83301739633649

$-0.91412428797793$

0.12960473308158

$-0.22308036856876$

$-0.73422931423874$

$-0.00927737405022$

2.57407798336239

$-0.11974626290297$

0.97284330993379

$-0.36706933986627$

$-0.48946714990227$

$-0.38038496994088$

$-0.83278833800094$

0.71122503196990

$-0.74635960175640$

$-2.20015435783330$

$-2.65423808014213$

$-2.62998269617389$

$-2.45623755305964$

I-8

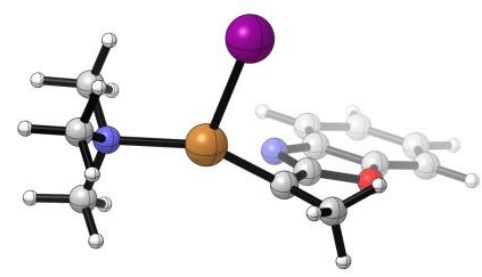

$\mathrm{E}_{\mathrm{SMD}}=-2950.2255 \mathrm{E}_{\mathrm{h}}$

$\mathrm{ZPE}=0.2980 \mathrm{E}_{\mathrm{h}}$

$\mathrm{G}_{298}=-2949.9789 \mathrm{E}_{\mathrm{h}}$

$\begin{array}{lr}\text { C } & -0.34957140131929 \\ \mathrm{C} & -1.70186517839745 \\ \mathrm{O} & -2.14138108534792 \\ \mathrm{~N} & 0.01086843730383 \\ \mathrm{C} & 0.37294437605084 \\ \mathrm{H} & 1.41811028818103 \\ \mathrm{C} & -2.39173644852196 \\ \mathrm{H} & -3.43633874403856\end{array}$

$-6.46459195135843$

$-6.52115994375776$

$-5.25124014238280$

$-5.14853877430373$

$-7.65479228667218$

$-7.62464201390092$

$-7.71443344174356$

$-7.73086711231478$
$-0.24967047143636$

0.16064607307406

0.29168355317351

$-0.38071213827261$

$-0.46154542918534$

$-0.77480151623016$

0.36746415017246

0.68216376478492 
$-8.88334058391759$

$-9.85074981836991$

$-8.85420500002664$

$-9.80078838363329$

$-4.44456657864001$

$-2.47543957165088$

$-2.98575981209470$

$-2.66524530713595$

$-1.39110603616604$

$-3.01630861352937$

$-1.02526039804818$

$-1.99556137396325$

$-1.79779276185199$

$-0.22539953000317$

0.45801338416496

1.66428079396361

1.63913586224869

0.48852466784730

2. 40091719015194

$-0.09667536725884$

0.43816600649543

$-0.65494487816903$

0.63520822706282

$-0.27766904938355$

0.49312346950290

$-0.95742999878061$

0.20748757586598

$-2.07120745186455$

$-1.63024015505708$

$-2.76549554357509$

$-2.64342836274890$
0.15379870421514

0.30627457325085

$-0.25372974303106$

$-0.40544711642073$

$-0.04715118203263$

0.41420928123588

1. 30347824348790

$-0.40177074906820$

0.58424947906599

$-0.02458187976155$

$-1.34791684868632$

$-0.08258379896550$

2.16728032835475

2. 21558094275203

1. 22509834025071

1.74976618114968

3.00562612936515

3. 38266830713379

3. 76083872296671

$-2.18443086419696$

$-2.90369638302911$

$-2.74837882802310$

$-1.53837909321631$

$-0.60874339705523$

0.00862046396296

0.05355507379156

$-1.30928928464290$

$-2.17727448758654$

$-2.92058395519047$

$-1.53859490851948$

$-2.71075327563745$
TS6 $^{\text {I }}$

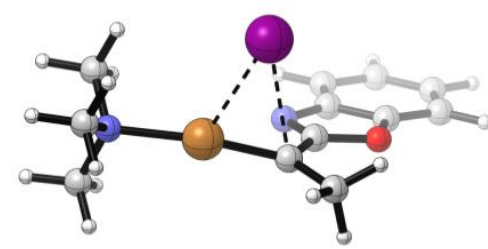

$\begin{array}{lr}\mathrm{C} & -0.18262492134204 \\ \mathrm{C} & -1.52486882951304 \\ \mathrm{O} & -2.11007156445797 \\ \mathrm{~N} & 0.02700565776294 \\ \mathrm{C} & 0.67142119092656 \\ \mathrm{H} & 1.71263824067246 \\ \mathrm{C} & -2.07943721290532 \\ \mathrm{H} & -3.12156159549022 \\ \mathrm{C} & -1.21509448815347 \\ \mathrm{H} & -1.59171844486392 \\ \mathrm{C} & 0.13467870176559 \\ \mathrm{H} & 0.76824355754462 \\ \mathrm{C} & -1.11312563027163 \\ \mathrm{C} & -2.85885929712975 \\ \mathrm{H} & -3.39324747581274 \\ \mathrm{H} & -3.35646803986382 \\ \mathrm{H} & -2.98985984484776 \\ \mathrm{C} & -1.41584943717597 \\ \mathrm{~N} & 1.72409053002918 \\ \mathrm{Cu} & 0.08413725394336\end{array}$

$$
\begin{aligned}
& E_{S M D}=-2588.7334 E_{h} \\
& Z P E=0.2609 E_{h} \\
& G_{298}=-2588.5187 E_{h}
\end{aligned}
$$

Imaginary frequency $=51.04 i \mathrm{~cm}^{-1}$
$-0.29065616276125$
0.10000453687577
0.17967886475089
$-0.45905456776950$
$-0.45024054383567$
$-0.74820858153388$
0.33410710028357
0.63343091370427
0.17283917814952
0.35086238679273
$-0.21300112372887$
$-0.32439671790432$
$-0.15853025620178$
$-0.03874329374787$
0.75254489406551
$-0.99741407912242$
0.11726288913779
$-0.19864235149202$
$-0.76593501370990$
$-0.32984691243396$ 

$-2.41718347812549$
0.38465251642077
0.33861151153787
1.01346240217725
0.85267106841668
$-1.61828687687860$
$-1.66915828428831$
$-2.64190528662379$
$-1.04297970787857$
$-0.93806600089381$
$-0.34534984516653$
$-1.95813387188823$
$-0.48184037664470$

2. 34084785661889

$-0.23674820842161$

0.84596992915997

$-0.42776573290758$

$-0.71999957145761$

$-0.09844991813094$

0.98428885781714

$-0.47749276447840$

$-0.28734751570553$

$-2.22571495802052$

$-2.47494668600936$

$-2.61413944226154$

$-2.72110988472149$

\section{TS6 $^{\mathrm{S}}$}

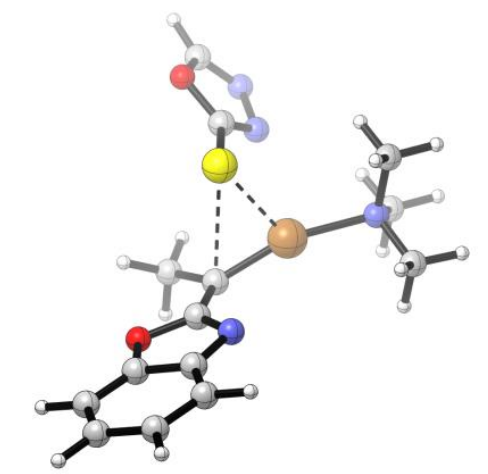

$$
\begin{aligned}
& E_{S M D}=-2950.2247 E_{h} \\
& Z P E=0.2980 E_{h} \\
& G_{298}=-2949.9765 E_{h} \\
& \text { Imaginary frequency }=37.58 i \mathrm{~cm}^{-1}
\end{aligned}
$$

\begin{tabular}{|c|c|}
\hline C & -0.40857459864682 \\
\hline C & -1.74321815116354 \\
\hline 0 & -2.26434537051589 \\
\hline$N$ & -0.14233839105439 \\
\hline C & 0.3841624430833 \\
\hline & 1.417439414719 \\
\hline$c$ & -2.3489444531543 \\
\hline $\mathrm{H}$ & -3.3833030519915 \\
\hline C & -1.54504372900900 \\
\hline$H$ & -1.96263791368870 \\
\hline C & -0.2044148560966 \\
\hline $\mathrm{H}$ & 0.37902067383831 \\
\hline C & -1.24107264966267 \\
\hline C & -2.82590386873978 \\
\hline $\mathrm{H}$ & -3.23977373464760 \\
\hline & -3.5125963439481 \\
\hline $\mathrm{H}$ & -2.8549355899343 \\
\hline C & -1.47209161357045 \\
\hline N & 1.57976828833330 \\
\hline $\mathrm{Cu}$ & 0.04842043919354 \\
\hline $\mathrm{S}$ & 0.08519994699776 \\
\hline C & -0.50858447838407 \\
\hline N & -0.6311847430526 \\
\hline $\mathrm{N}$ & -1.1657190919401 \\
\hline C & -1.3282014709788 \\
\hline & -0.9466153826518 \\
\hline$H$ & -1.7150021889273 \\
\hline & 0.9929695598220 \\
\hline & 1.7810990511839 \\
\hline & 0.2687855134554 \\
\hline & $0.471628885921 €$ \\
\hline & 2.5606657531388 \\
\hline & 2.061788638764 \\
\hline
\end{tabular}

$-6.60194452482166$

$-6.72364217800173$

$-5.47723757503405$

$-5.27253105005560$

$-7.75310191629124$

$-7.67505907027587$

$-7.94431349281721$

$-8.00893489482746$

$-9.07597613117638$

$-10.06183645639192$

$-8.98156786758125$

$-9.89874905708996$

$-4.62539305291984$

$-2.73493565628797$

$-3.25123031312718$

$-2.95507730499386$

$-1.64714519500896$

$-3.20788205230466$

$-1.00629161984366$

$-2.11569569498241$

$-2.43506701607720$

$-0.83084761154814$

0.12381178614138

1.22816970684400

0.87931634242111

$-0.39879934564951$

1.47033690156485

0.22075563150813

0.85773023300468

$-0.04859912945049$

0.78225133267264

$-0.64200200120825$

$-0.05292438181796$
$-0.27257793083986$

0.17556334904812

0.24559897437448

$-0.49392610643734$

$-0.43483831832532$

$-0.77814273457538$

0.46426145963777

0.80574613786494

0.30010323912074

0.51983449265477

$-0.14298477703593$

$-0.25577988858193$

$-0.16794451355442$

0.18719716171002

1.07043281049225

$-0.65592293642054$

0.33829462548883

$-0.23545959365483$

$-1.22363496677920$

$-0.41615583935102$

2.00471485274039

2. 21932781715025

1. 32527506417970

1.97295616127289

3. 20422480985731

3.44391233596703

4.03191495931574

$-1.80430731016994$

$-2.25348001295409$

$-2.58667172396807$

$-1.01709680457865$

$-0.18122040868796$

0.59900675837386 


$\begin{array}{ll}\mathrm{H} & 2.97387112489423 \\ \mathrm{H} & 3.38904197007178 \\ \mathrm{C} & 2.23139309076693 \\ \mathrm{H} & 3.05850752194633 \\ \mathrm{H} & 2.63798877998739 \\ \mathrm{H} & 1.49712234264012\end{array}$

\section{I-9 ${ }^{\text {I }}$}

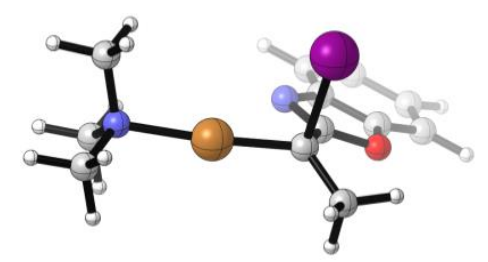

C $\quad 6.84399860848675$

$0 \quad 6.78321245662641$

$\mathrm{N} \quad 4.72466023049250$

C $\quad 5.32344516555222$

$\mathrm{H} \quad 4.33084918260764$

C $\quad 7.93550345412004$

H $\quad 8.92223608982318$

C $\quad 7.68751802103589$

$\mathrm{H} \quad 8.50556244067207$

C $\quad 6.40532376909510$

H $\quad 6.25815757038980$

C $\quad 5.46941313640291$

C $\quad 6.22103313451358$

$\mathrm{H} \quad 7.02770725171567$

$\mathrm{H} \quad 6.66379336154752$

H $\quad 5.86863926403488$

C $\quad 5.07073440430112$

$\mathrm{N} \quad 1.60947434550249$

$\mathrm{Cu} \quad 3.40371938281906$

I $\quad 4.22600908966409$

C $\quad 1.61156792390703$

$\mathrm{H} \quad 1.83543530953231$

$\mathrm{H} \quad 2.38358115677217$

$\mathrm{H} \quad 0.62762400588629$

C $\quad 0.57697210004511$

H $\quad 0.59364933565892$

$\mathrm{H} \quad 0.78036904134816$

$\mathrm{H} \quad-0.43170993153481$

C $\quad 1.35537516756674$

$\mathrm{H} \quad 0.36587433659373$

$\mathrm{H} \quad 2.13078594690489$

H $\quad 1.37673557256790$
$-1.55066954593021$

$-0.04687523234553$

$-1.81103474206821$

$-1.24746767930980$

$-2.73644388580342$

$-2.08128581111378$
0.27813307815410

$-0.61390627348148$

$-2.27584827584754$

$-2.75242856500471$

$-1.84306227668121$

$-3.04802864747374$
$-5.35602140801453$

$-5.16375330259105$

$-4.08052421970233$

$-4.36570653947549$

$-6.41151667452699$

$-6.57784261724864$

$-5.96855093460320$

$-5.79295906060275$

$-7.02017006771593$

$-7.69075343535882$

$-7.23584204307349$

$-8.07051787387554$

$-3.64879848058118$

$-1.55988014873611$

$-2.07702021953620$

$-1.13244532215276$

$-0.72193063657867$

$-2.46821886429814$

$-1.29101439551416$

$-1.80020918106379$

$-3.34986667768612$

$-1.65550199631957$

$-2.72551254959729$

$-1.08059387811354$

$-1.44177025234886$

$-2.07078366319526$

$-1.81847623345012$

$-3.14557751014722$

$-1.85495589062493$

0.15505527647647

0.43132115630406

0.73127815256454

0.42570689838768
$-0.31118626169960$

$-0.84568064441484$

$-1.66845864497513$

$-0.80830592231771$

0.58294492076626

1.00649651637632

$-0.53652301204722$

$-0.96869594506311$

0.35452300782761

0.62945643746572

0.90324155113043

1.59375951477991

$-1.60873617253852$

$-2.79100764310328$

$-3.33842560892526$

$-1.87196101809706$

$-3.40872259231517$

$-2.38762786950851$

$-0.90905864962012$

$-1.67597791908496$

$-4.38559891573032$

0.52430127677428

0.63652538293351

1.05524356378294

0.98717192991000

$-1.62656333364950$

$-2.69612775869252$

$-1.52093288324135$

$-1.22180522091156$

$-1.06612712398235$

$-0.65016320682003$

$-0.54149373247203$

$-2.13139249053679$ 
I-9

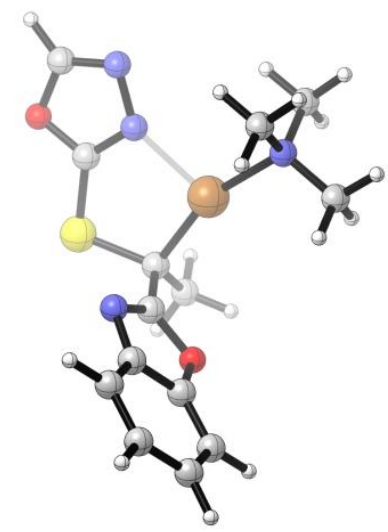

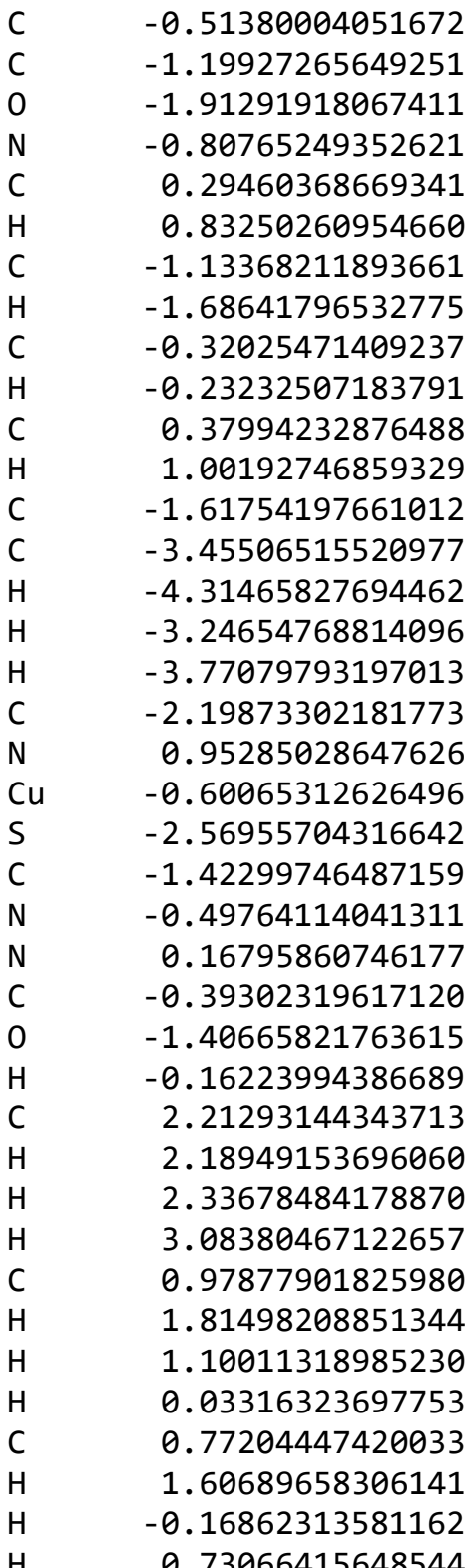

$E_{S M D}=-2950.2559 E_{h}$

$\mathrm{ZPE}=0.2998 \mathrm{E}_{\mathrm{h}}$

$\mathrm{G}_{298}=-2950.0061 \mathrm{E}_{\mathrm{h}}$
$-6.41736440417958$

$-6.64117382650010$

$-5.50600435074162$

$-5.13896588328970$

$-7.43148883036970$

$-7.28152474980677$

$-7.82529705676285$

$-7.96507758548865$

$-8.83288132083395$

$-9.78883945899961$

$-8.63519121292608$

$-9.44515811795930$

$-4.63414208309340$

$-3.18034975356600$

$-3.75684531423584$

$-3.55967623831576$

$-2.13097780690610$

$-3.29128747184370$

$-1.77680945507527$

$-2.27822629066037$

$-2.65538922746268$

$-1.36545766202092$

$-0.97593112782513$

0.09182336578587

0.27387745372046

$-0.60612649048772$

1.01109272659640

$-1.92032499498448$

$-1.27414411109814$

$-2.96297413537099$

$-1.63887827925677$

$-2.64888784285243$

$-2.37573871447408$

$-3.69740045990834$

$-2.55649801160163$

$-0.36928599871469$

$-0.03146057648229$

$-0.26006580216267$

0.27305561315478
0.76486275392506

$-0.44404532131793$

$-0.71995164043207$

1. 21353447864419

1. 29557003250752

2. 23401254460141

$-1.16476272167259$

$-2.09586593746382$

$-0.62124424458839$

$-1.14361779033921$

0.58387469594547

0.97419339404003

0.31819158868493

$-0.65287981697619$

$-0.25907778092438$

$-1.66426550602358$

$-0.74750979002065$

0.22270008393080

$-1.79166877390206$

$-0.52984206051760$

1. 91341749695529

2.06392547687066

1. 22218873694705

1.80902851459623

2.95316610498822

3. 18625420985112

3. 71920689256452

$-1.03401886166760$

$-0.14558412707432$

$-0.70801023946990$

$-1.66013364736860$

$-2.98123467984932$

$-3.65724828729354$

$-2.67497580095629$

$-3.53392647649980$

$-2.19787555505660$

$-2.84485473599478$

$-2.75711258426409$

$-1.30699583637925$ 


\section{Copies of NMR spectra}

\section{1-Iodo-5-methylhex-1-yne (S1a)}

${ }^{1} \mathrm{H}$ NMR (400 MHz, $\mathrm{CDCl}_{3}$ )

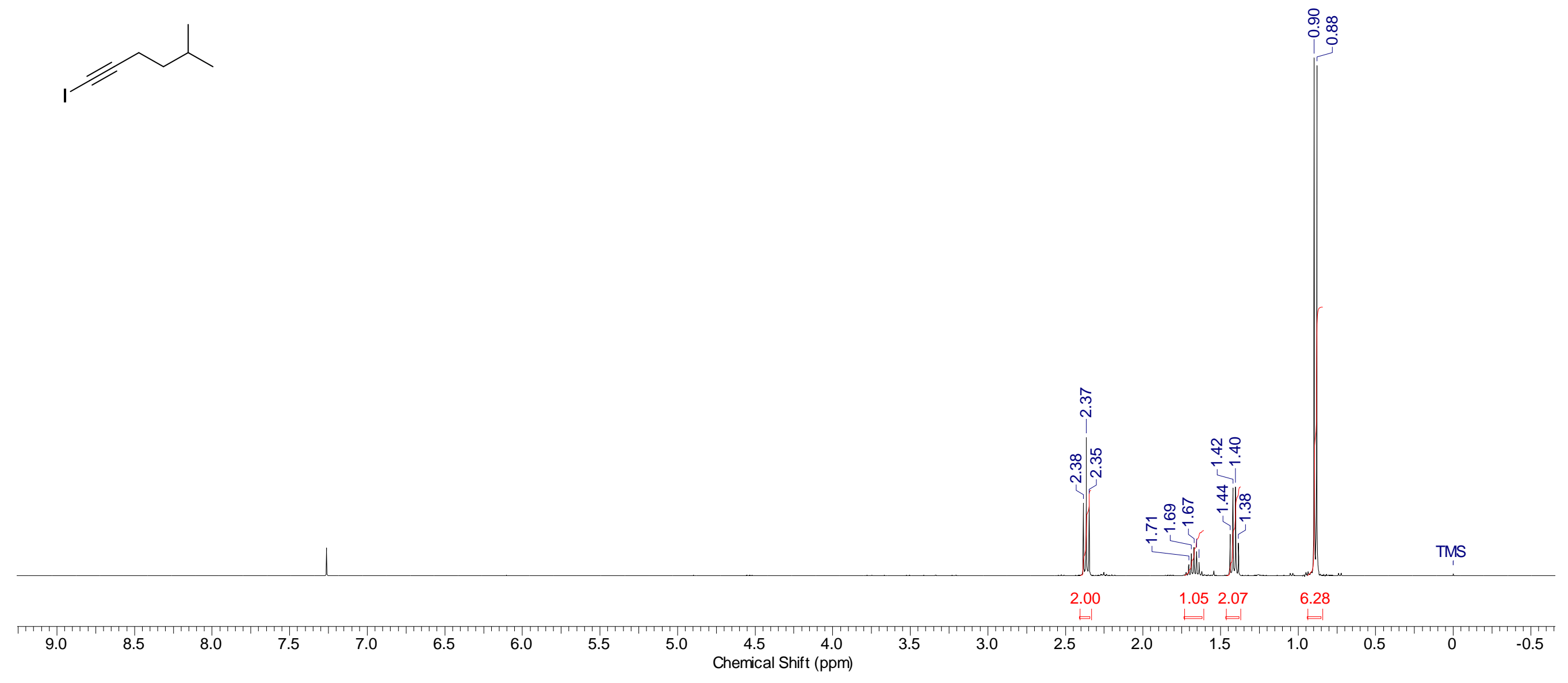




\section{1-Iodo-5-methylhex-1-yne (S1a)}

${ }^{13} \mathrm{C}$ NMR (100.6 MHz, $\mathrm{CDCl}_{3}$ )

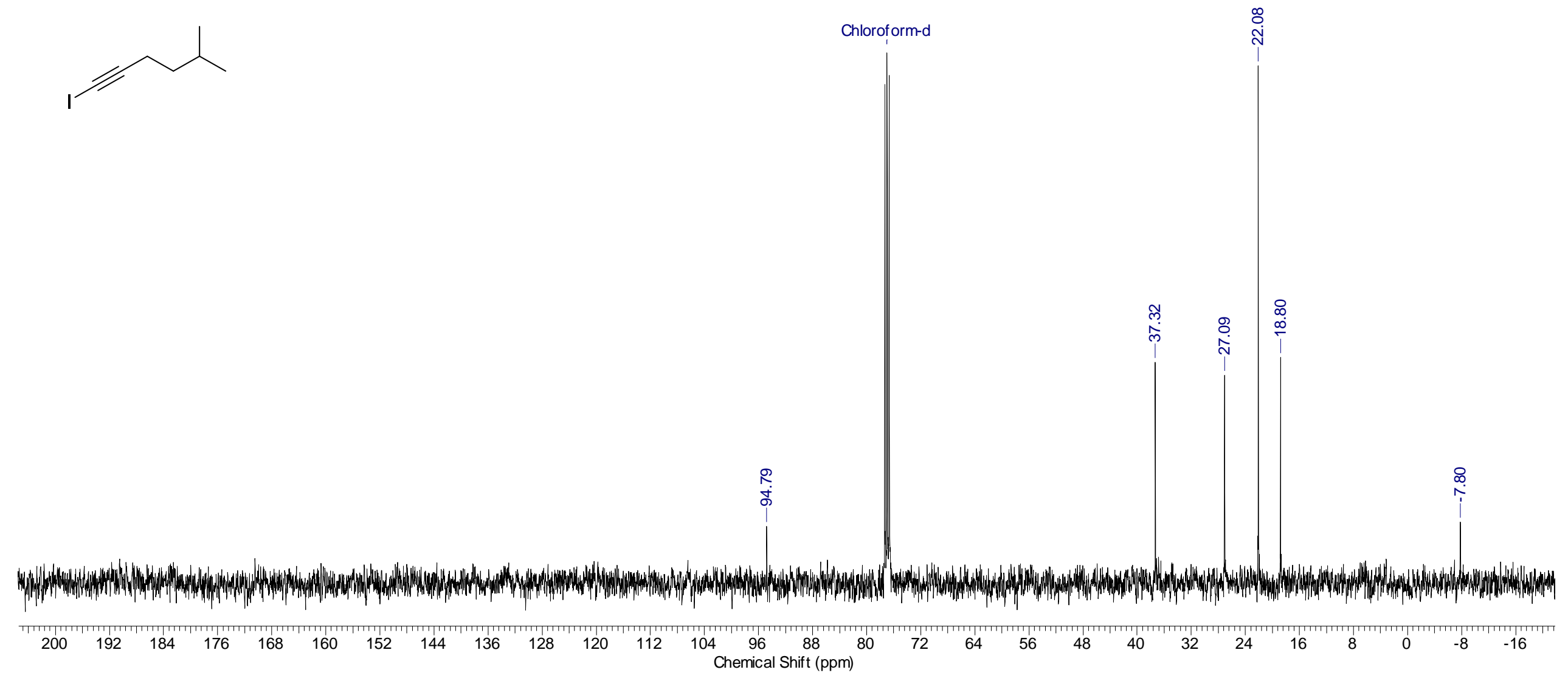




\section{(Iodoethynyl)cyclohexane (S1b)}

${ }^{1} \mathrm{H}$ NMR (400 MHz, $\mathrm{CDCl}_{3}$ )

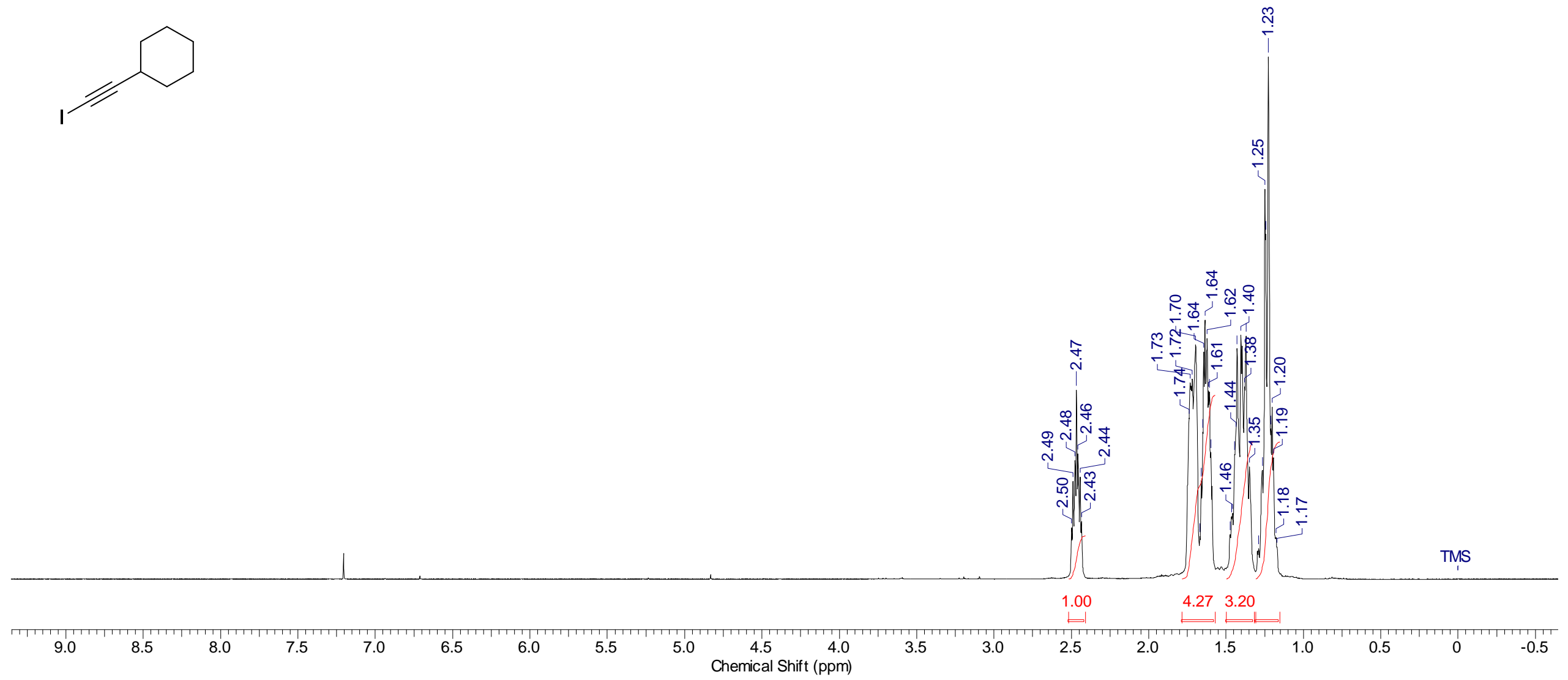




\section{(3-Iodoprop-2-yn-1-yl)cyclohexane (S1c)}

${ }^{1} \mathrm{H}$ NMR (600 MHz, $\mathrm{CDCl}_{3}$ )

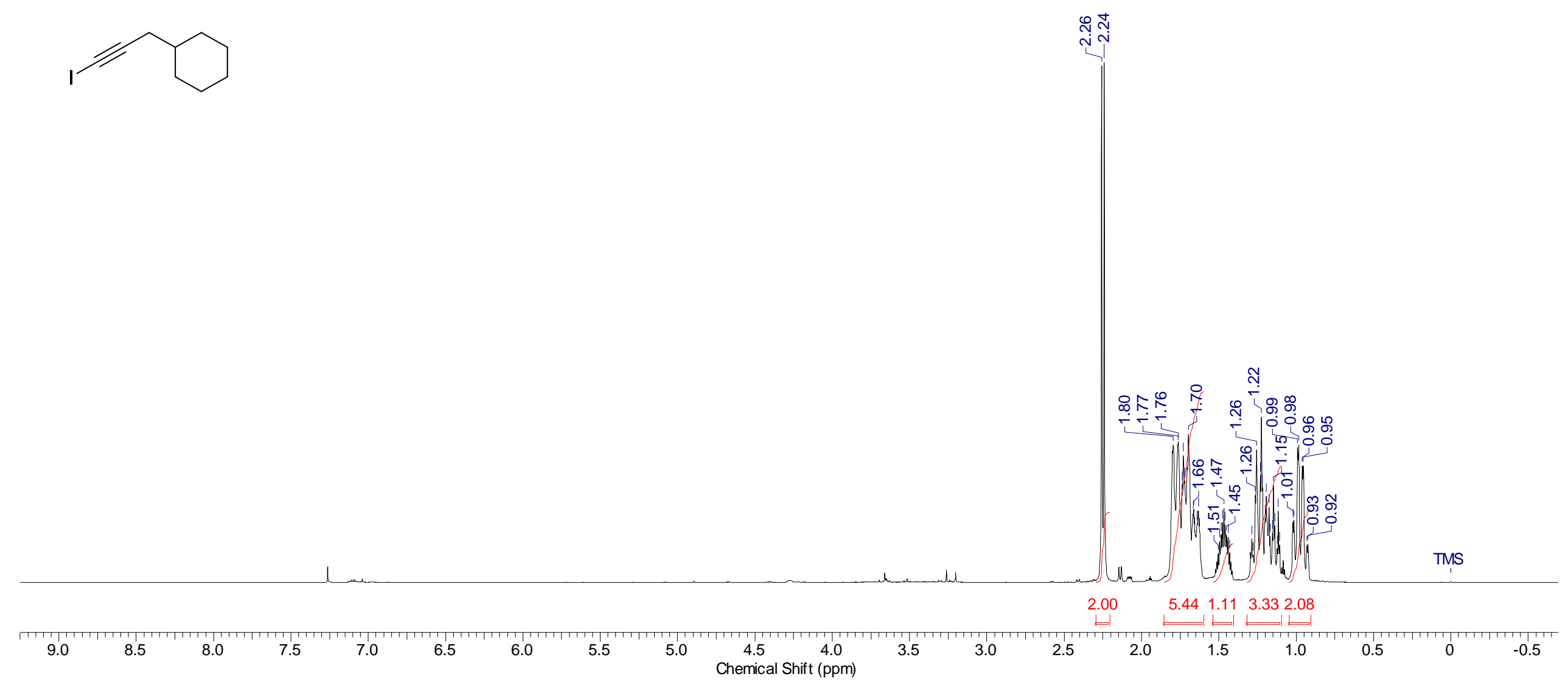




\section{(3-Iodoprop-2-yn-1-yl)cyclohexane (S1c)}

${ }^{13} \mathrm{C}$ NMR (151 MHz, $\mathrm{CDCl}_{3}$ )
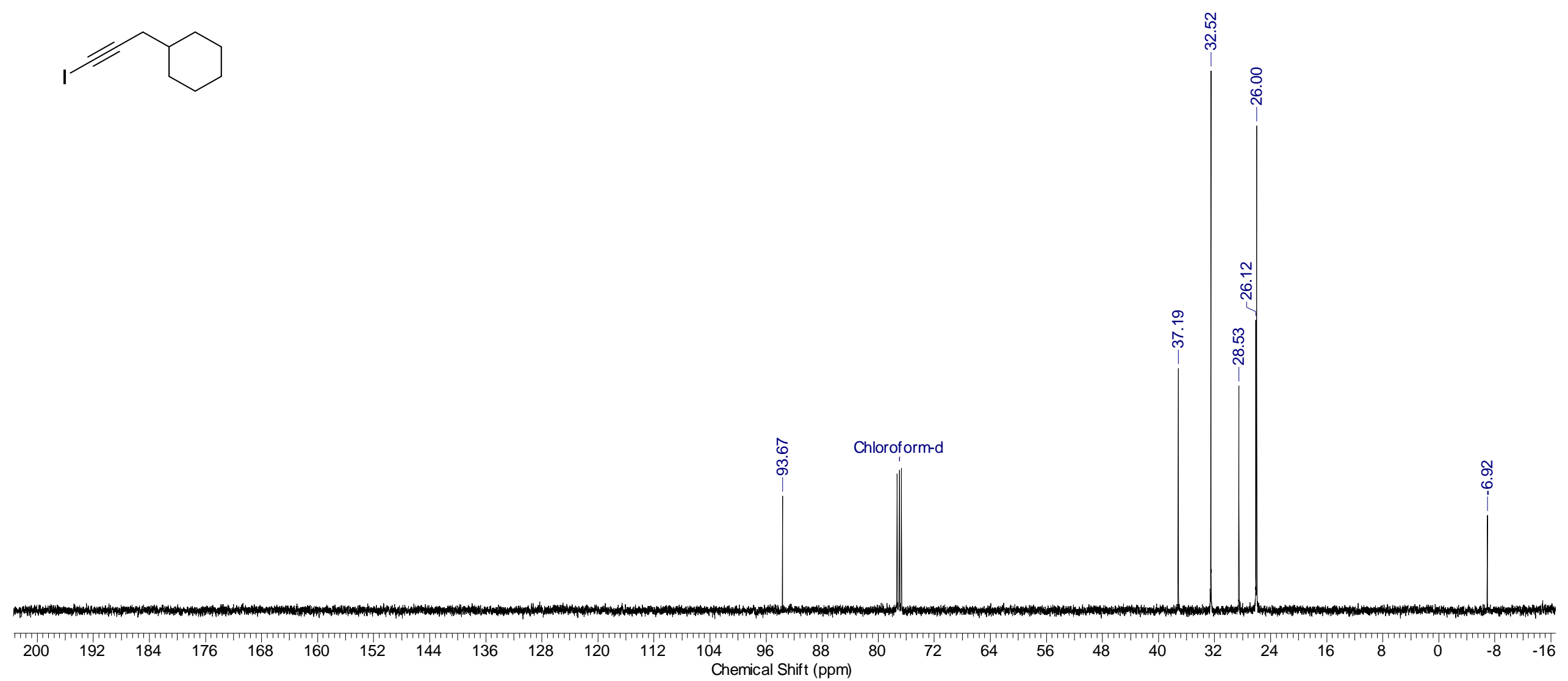


\section{(3-Iodoprop-2-yn-1-yl)benzene (S1d)}

${ }^{1} \mathrm{H}$ NMR (600 MHz, $\mathrm{CDCl}_{3}$ )

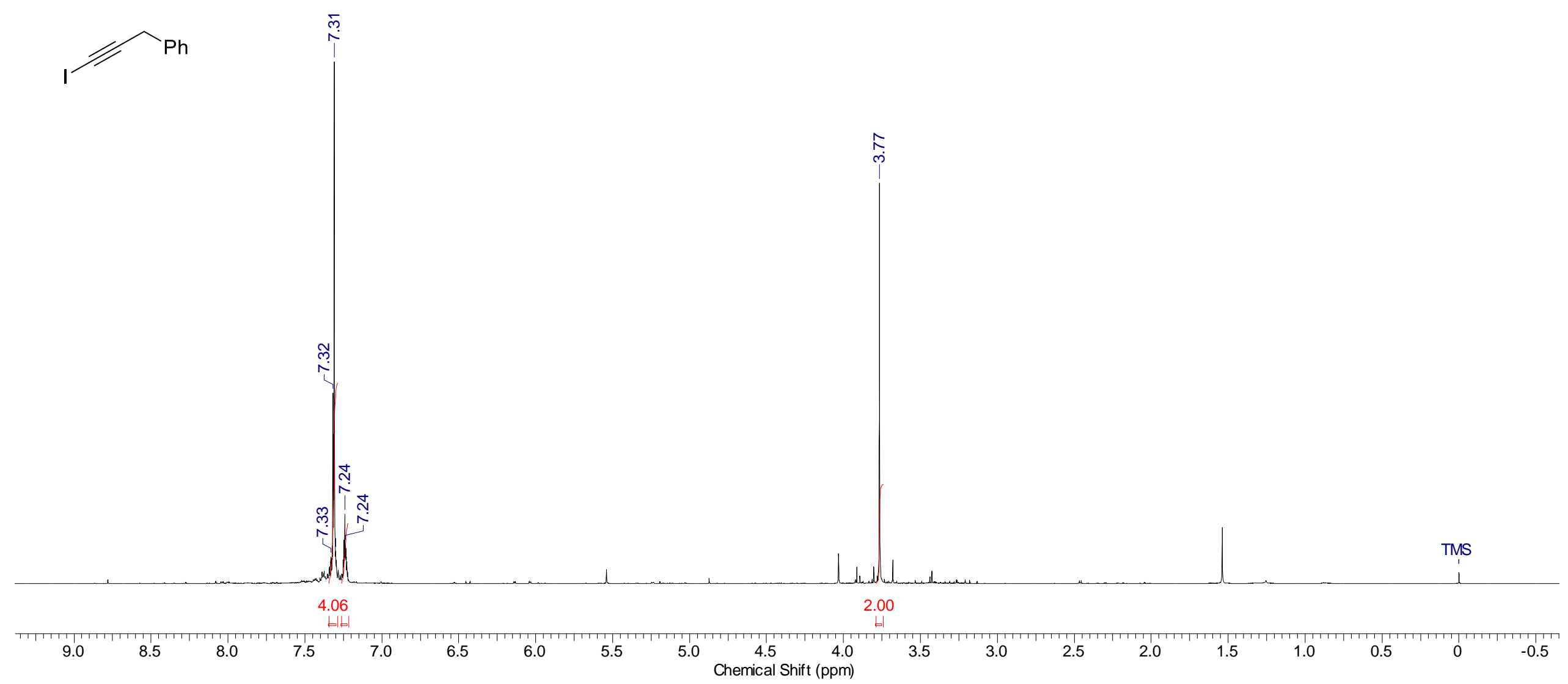


(3-Iodoprop-2-yn-1-yl)benzene (S1d)

${ }^{13} \mathrm{C}$ NMR (151 MHz, $\mathrm{CDCl}_{3}$ )

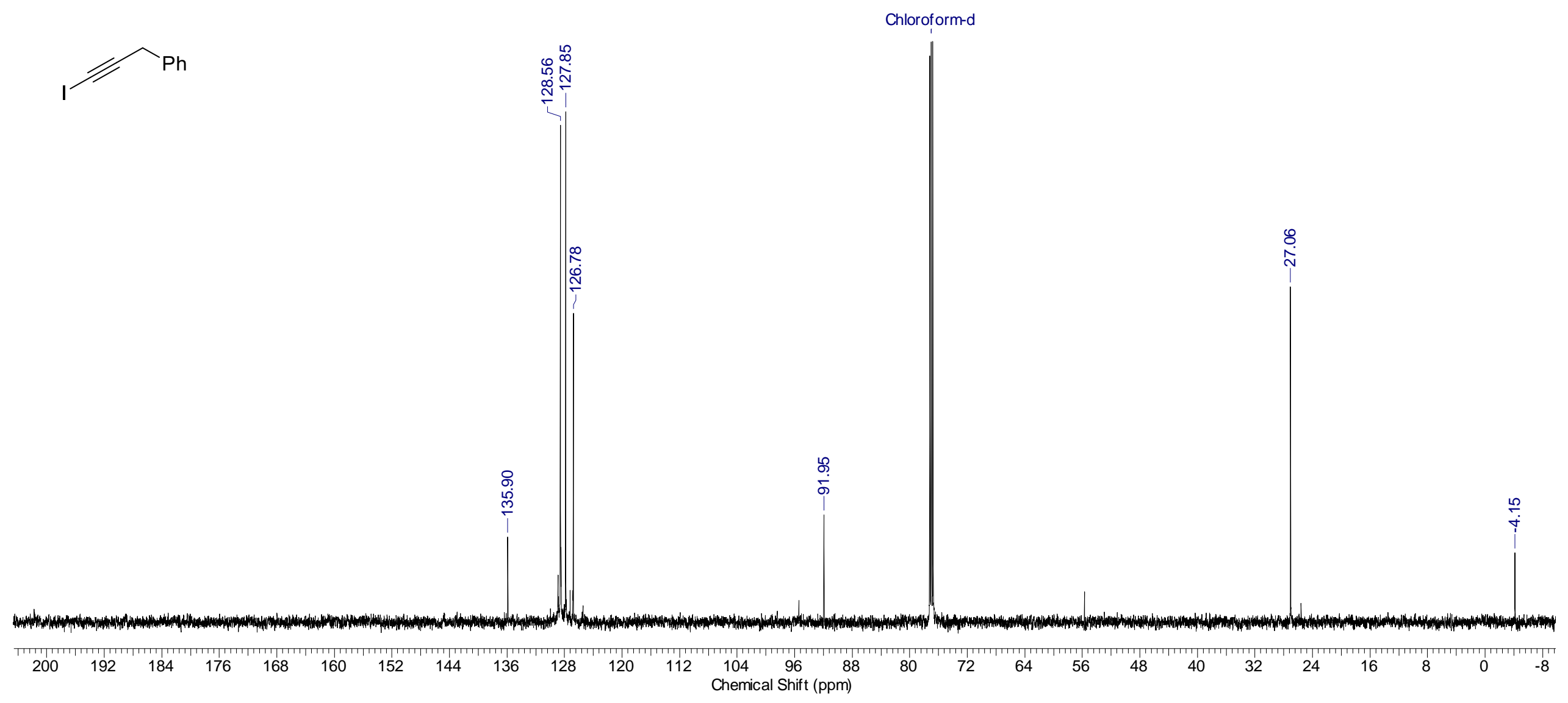


Dimethyl (3-iodoprop-2-yn-1-yl)(methyl)malonate (S1e)

${ }^{1} \mathrm{H}$ NMR (400 MHz, $\left.\mathrm{CDCl}_{3}\right)$

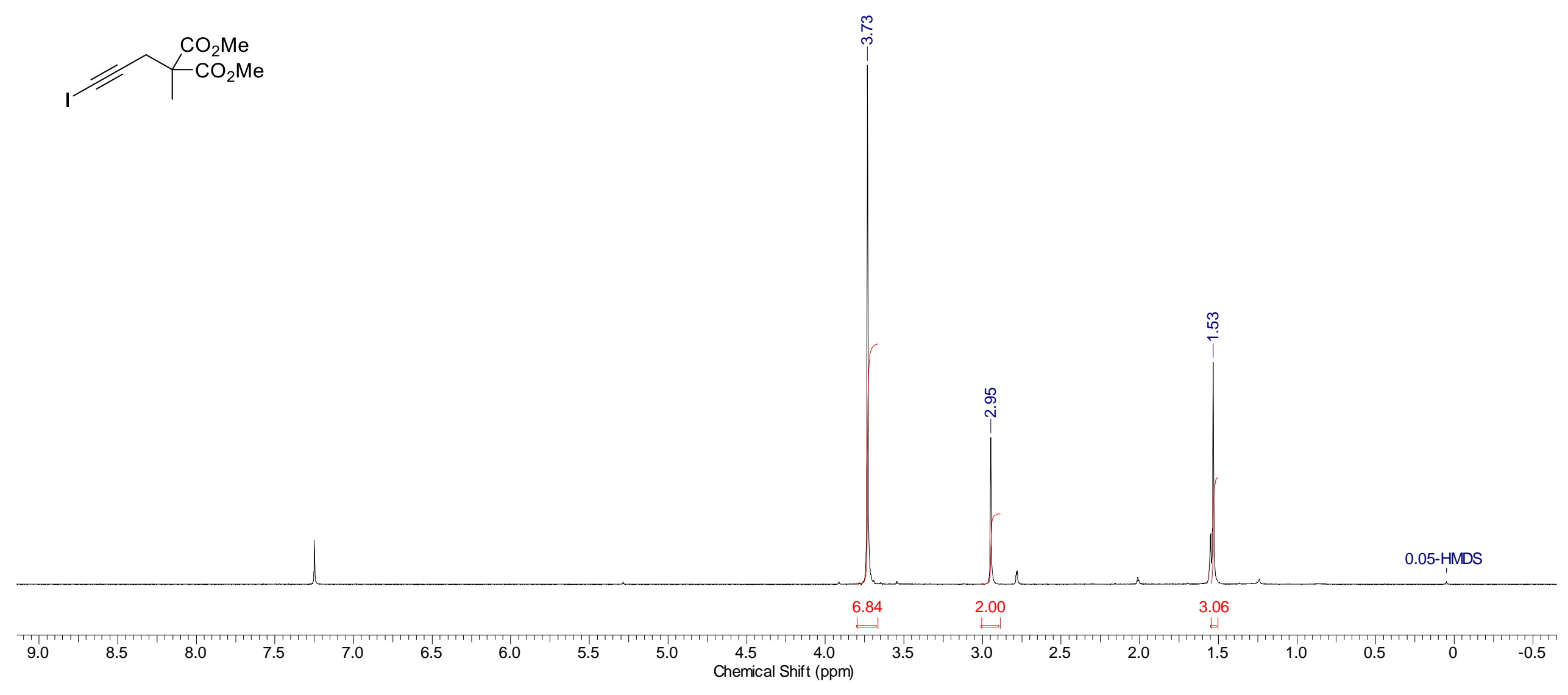


Dimethyl (3-iodoprop-2-yn-1-yl)(methyl)malonate (S1e)

${ }^{13} \mathrm{C}$ NMR (100.6 MHz, $\mathrm{CDCl}_{3}$ )

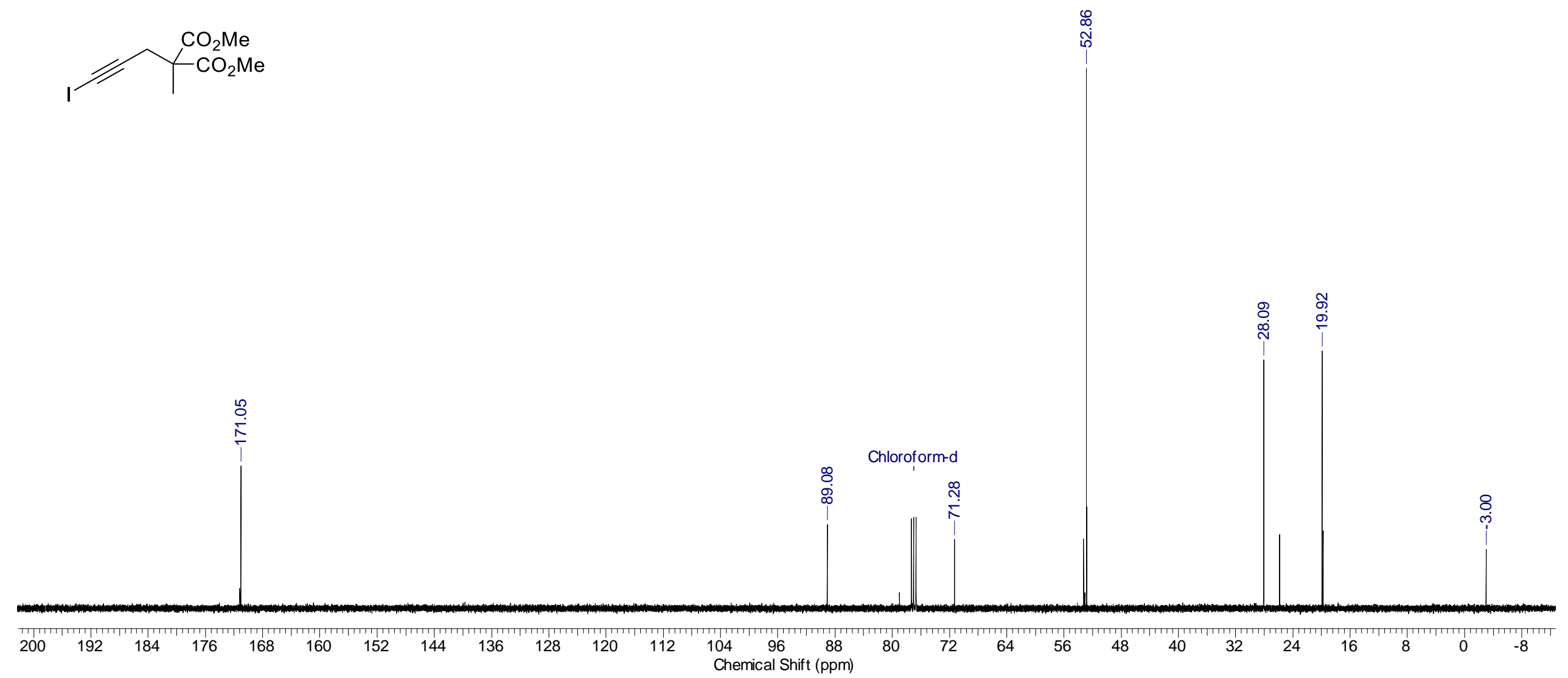


2-[5-Iodo-4-(3-methylbutyl)-1H-1,2,3-triazol-1-yl]phenyl acetate (S2h)

${ }^{1} \mathrm{H}$ NMR (400 MHz, $\mathrm{CDCl}_{3}$ )

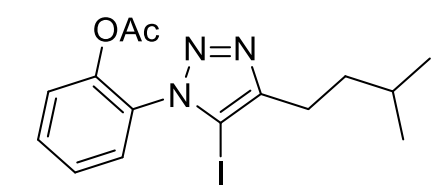

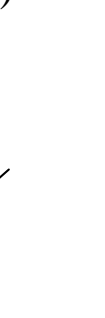


2-[5-Iodo-4-(3-methylbutyl)-1H-1,2,3-triazol-1-yl]phenyl acetate (S2h)

${ }^{13} \mathrm{C}$ NMR (100.6 MHz, $\left.\mathrm{CDCl}_{3}\right)$
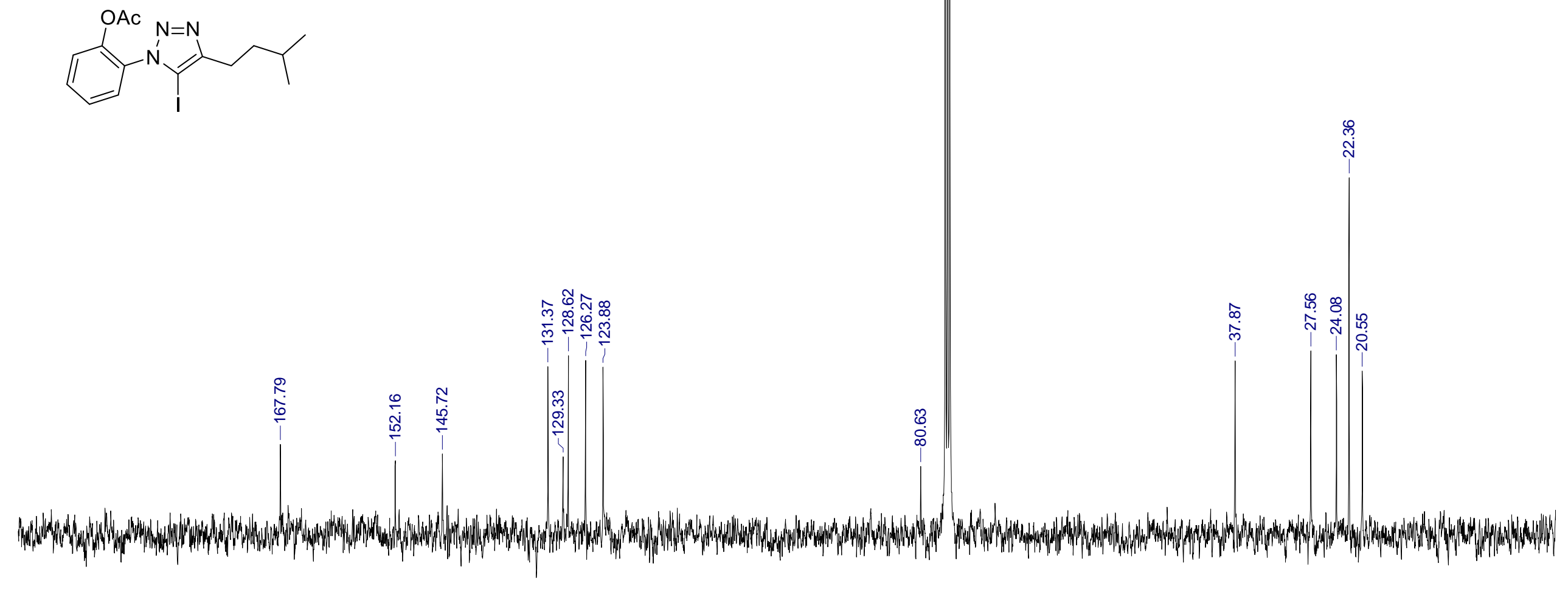

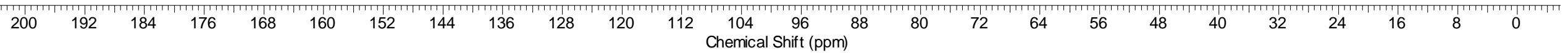




\section{2-(4-Cyclohexyl-5-iodo-1H-1,2,3-triazol-1-yl)phenyl acetate (S2j)}

${ }^{1} \mathrm{H}$ NMR (400 MHz, $\mathrm{CDCl}_{3}$ )
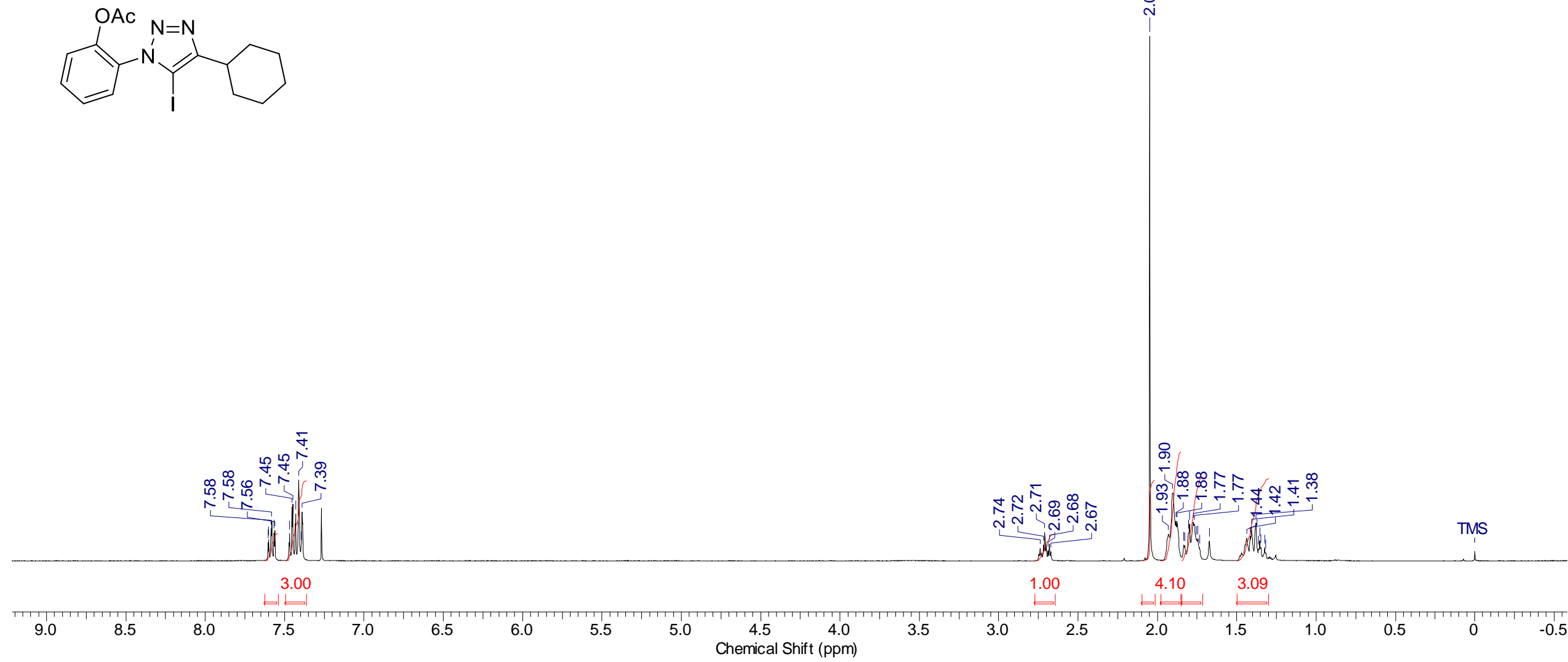
${ }^{13} \mathrm{C}$ NMR (100.6 MHz, $\left.\mathrm{CDCl}_{3}\right)$

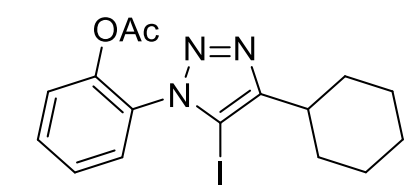

$$
\text { Chloroform-d }
$$

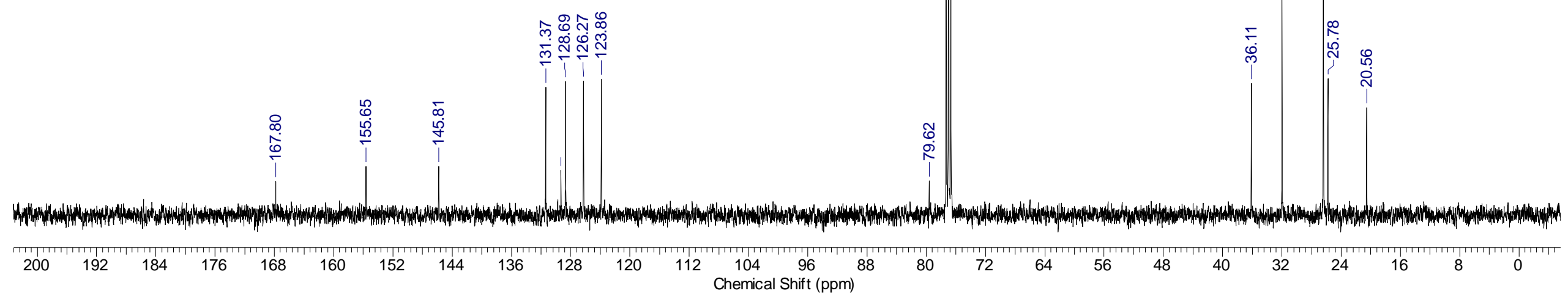




\section{2-[4-(Cyclohexylmethyl)-5-iodo-1H-1,2,3-triazol-1-yl]phenyl acetate (S2k)}

${ }^{1} \mathrm{H}$ NMR (600 MHz, $\mathrm{CDCl}_{3}$ )

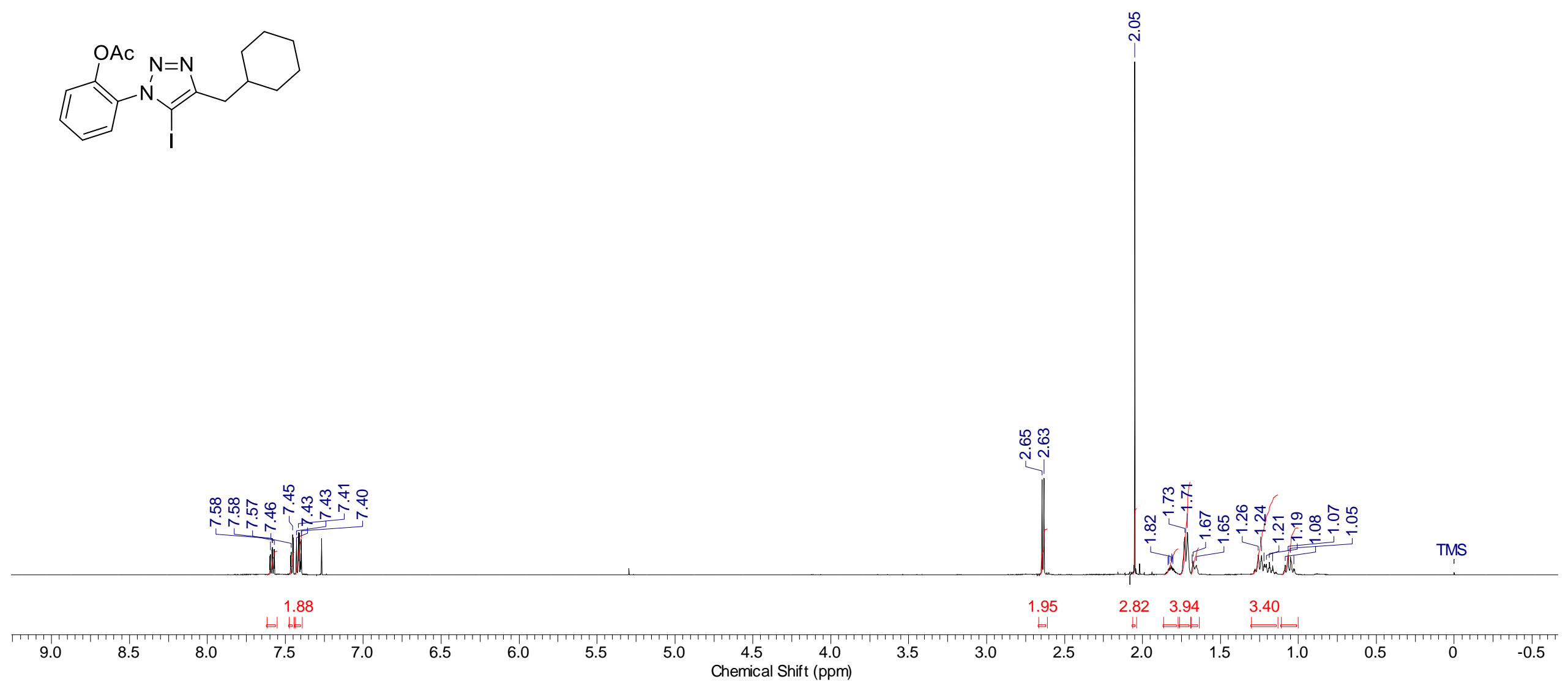


2-[4-(Cyclohexylmethyl)-5-iodo-1H-1,2,3-triazol-1-yl]phenyl acetate (S2k)

${ }^{13} \mathrm{C}$ NMR (151 MHz, $\left.\mathrm{CDCl}_{3}\right)$
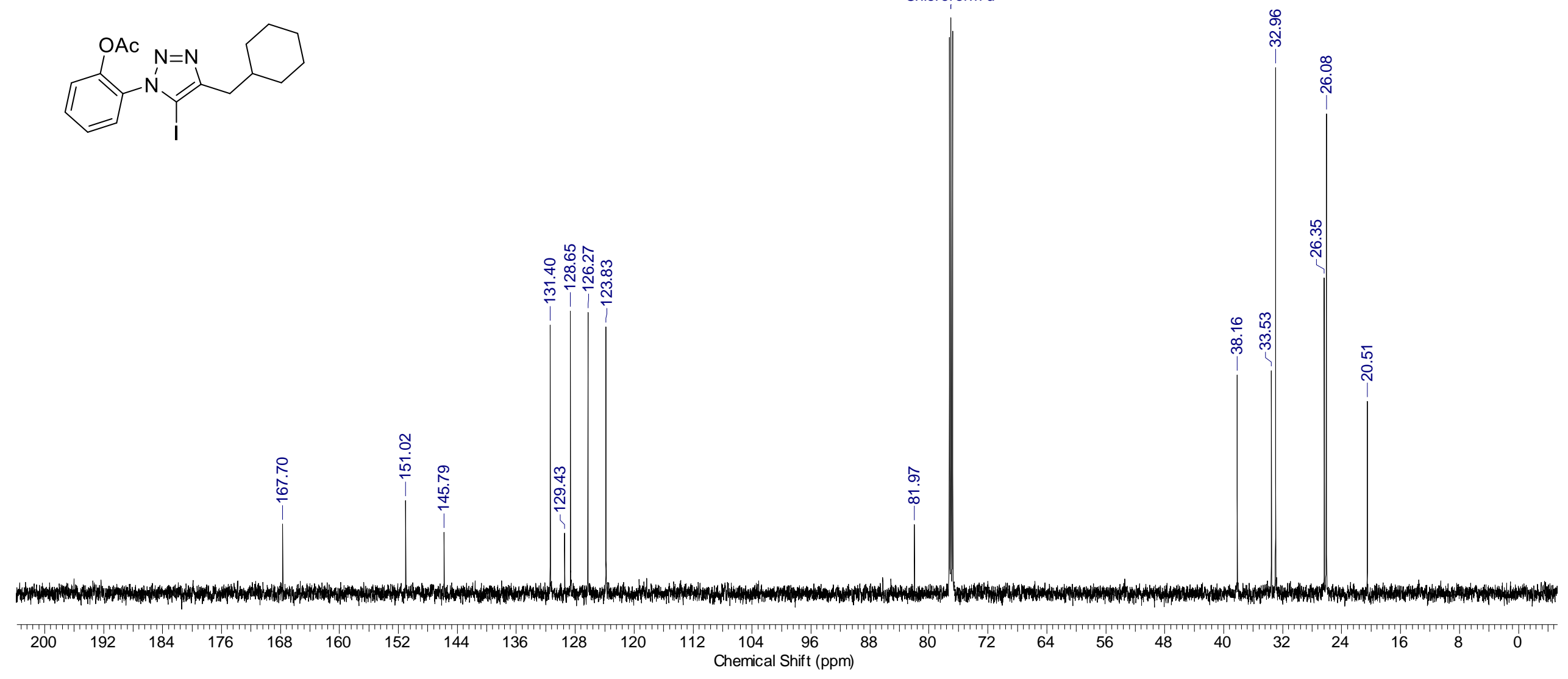


\section{2-(4-Benzyl-5-iodo-1H-1,2,3-triazol-1-yl)phenyl acetate (S2I)}

${ }^{1} \mathrm{H}$ NMR (400 MHz, $\mathrm{CDCl}_{3}$ )
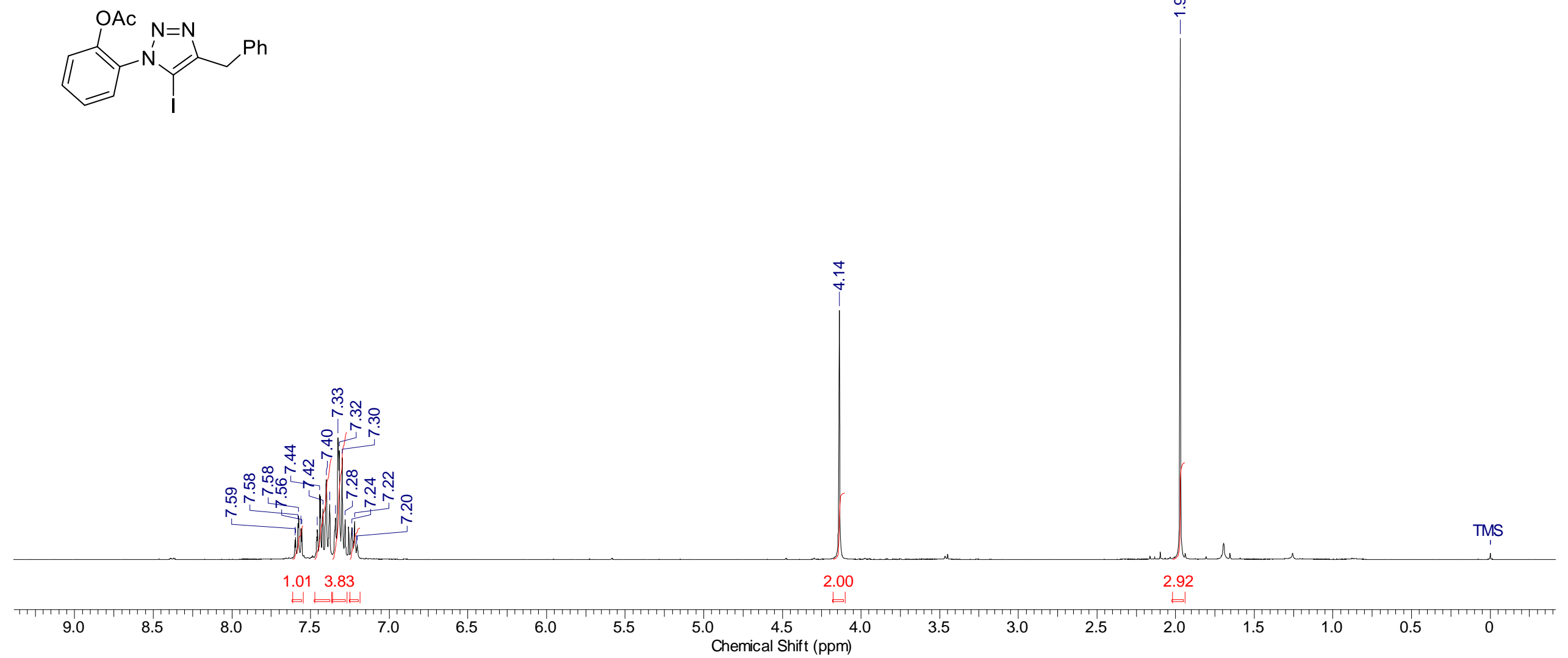


\section{2-(4-Benzyl-5-iodo-1H-1,2,3-triazol-1-yl)phenyl acetate (S2I)}

${ }^{13} \mathrm{C}$ NMR (100.6 MHz, $\mathrm{CDCl}_{3}$ )

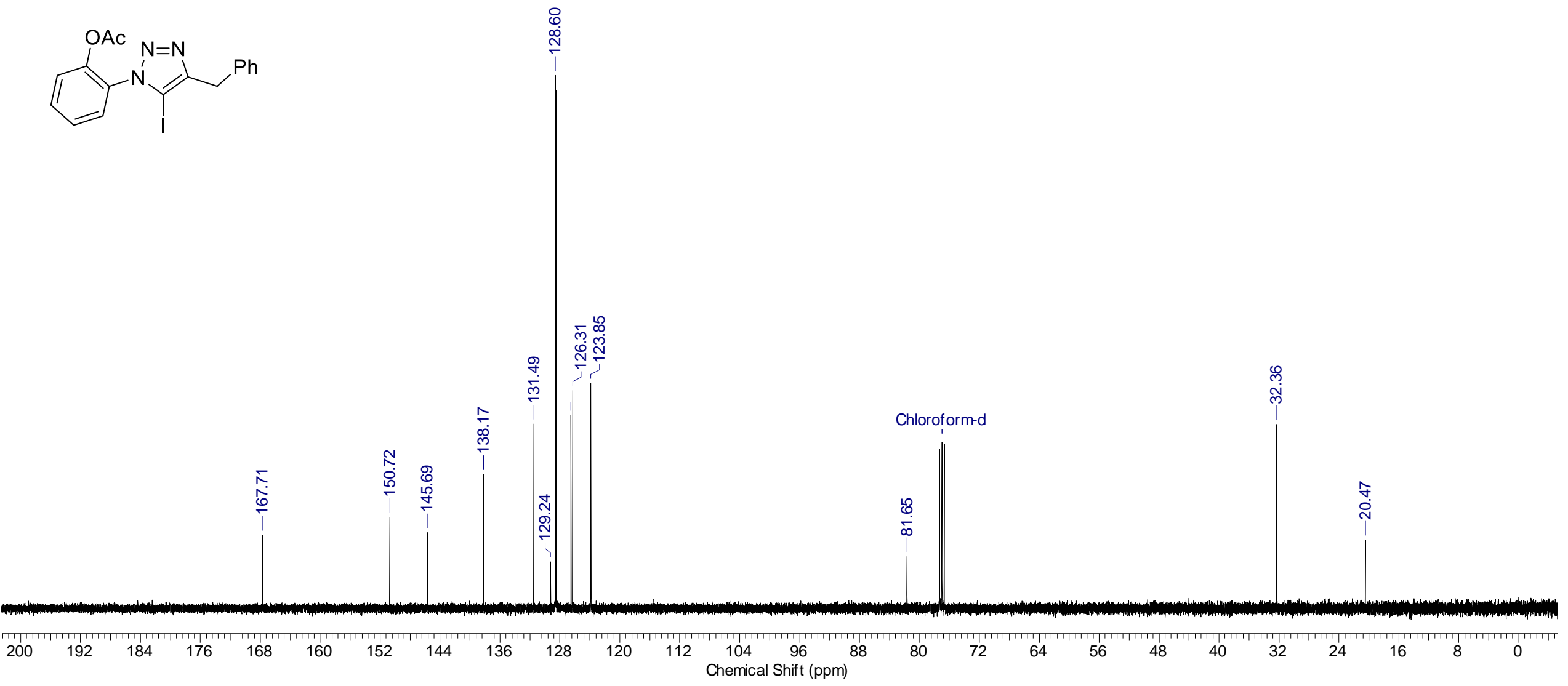




\section{2-[4-(Chloromethyl)-5-iodo-1H-1,2,3-triazol-1-yl]phenyl acetate (S2n')}

${ }^{1} \mathrm{H}$ NMR (400 MHz, $\mathrm{CDCl}_{3}$ )

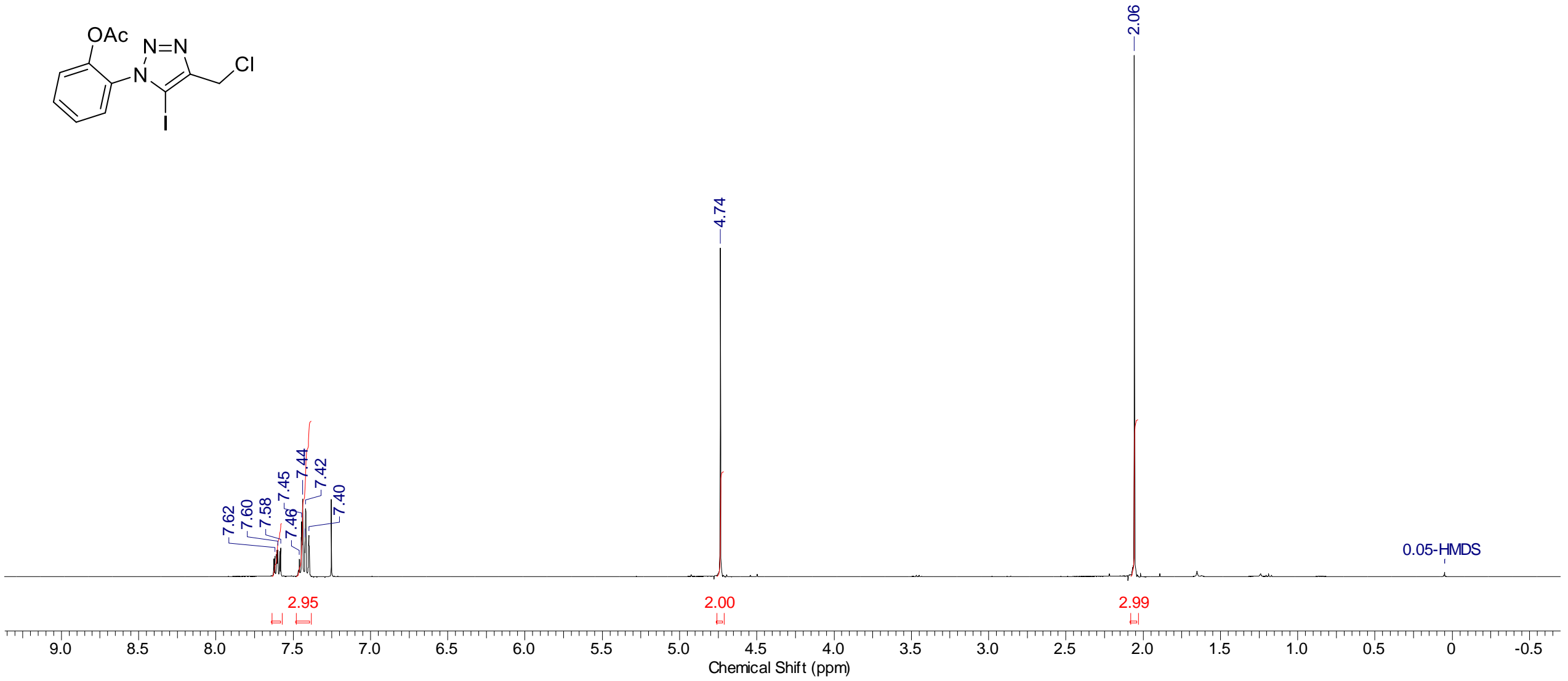




\section{2-[4-(Chloromethyl)-5-iodo-1H-1,2,3-triazol-1-yl]phenyl acetate (S2n')}

${ }^{13} \mathrm{C}$ NMR (100.6 MHz, $\left.\mathrm{CDCl}_{3}\right)$

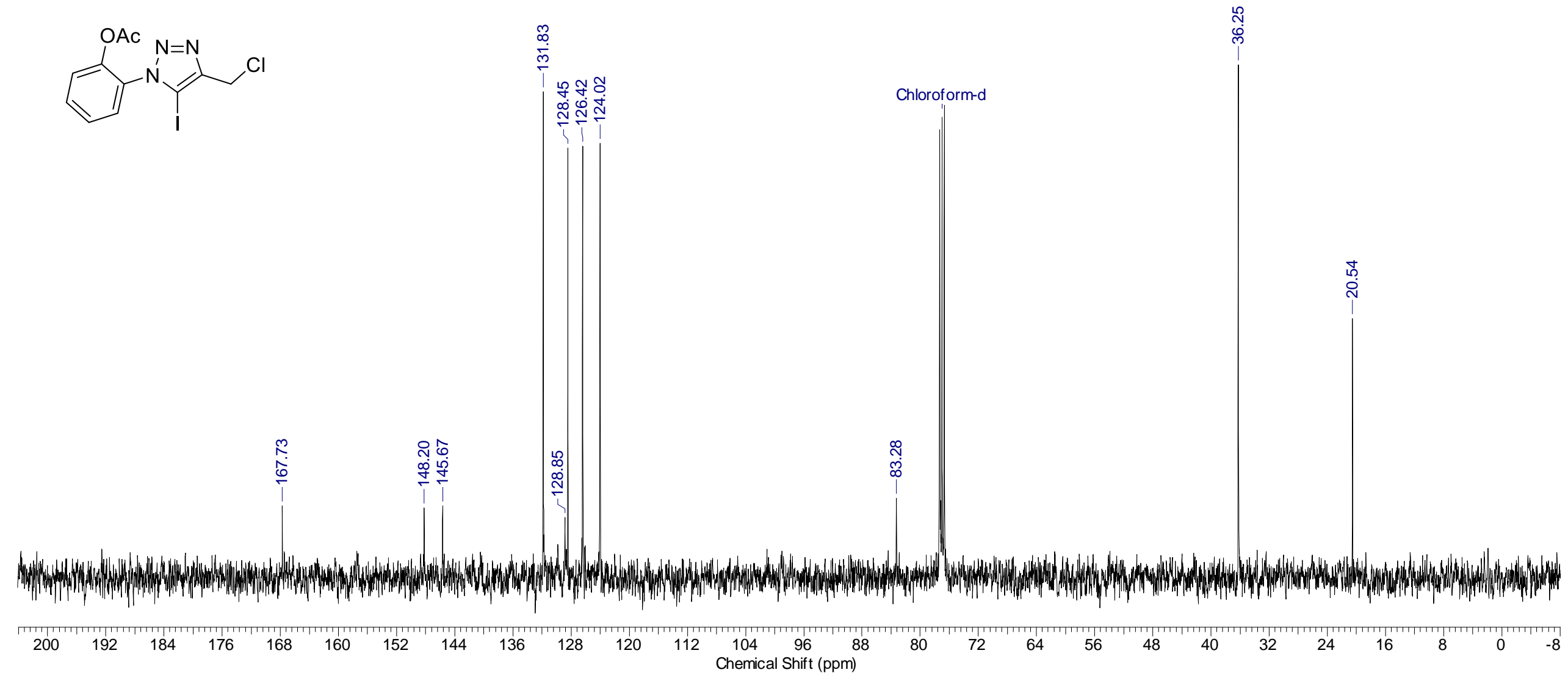


2-[4-(Azidomethyl)-5-iodo-1H-1,2,3-triazol-1-yl]phenyl acetate (S2n)

${ }^{1} \mathrm{H}$ NMR (400 MHz, $\left.\mathrm{CDCl}_{3}\right)$

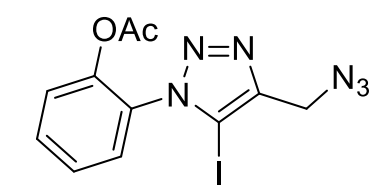

)


2-[4-(Azidomethyl)-5-iodo-1H-1,2,3-triazol-1-yl]phenyl acetate (S2n)

${ }^{13} \mathrm{C}$ NMR (100.6 MHz, $\mathrm{CDCl}_{3}$ )

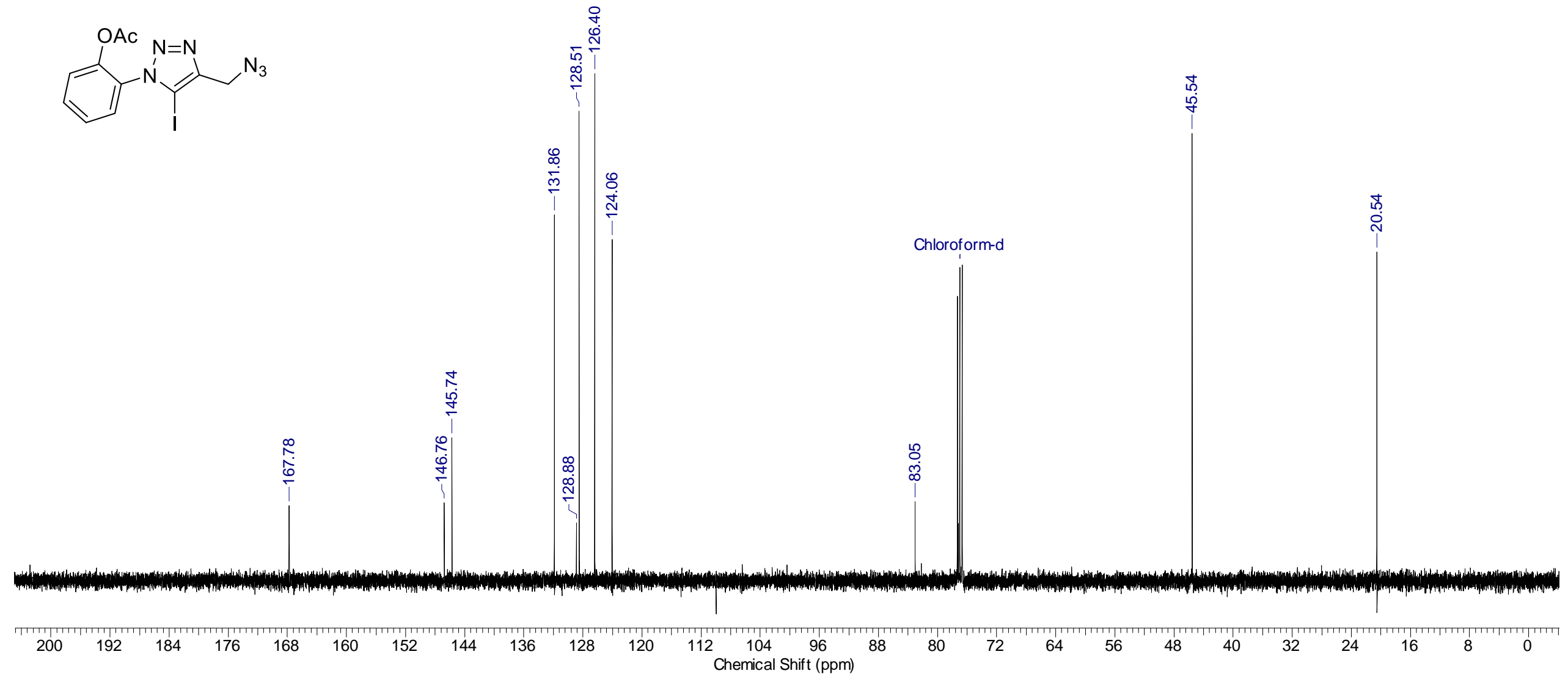


Dimethyl $\{[1-(2-a c e t o x y p h e n y l)-5-i o d o-1 H-1,2,3-t r i a z o l-4-y l] m e t h y l\}($ methyl)malonate (S2o)

${ }^{1} \mathrm{H}$ NMR (400 MHz, $\left.\mathrm{CDCl}_{3}\right)$

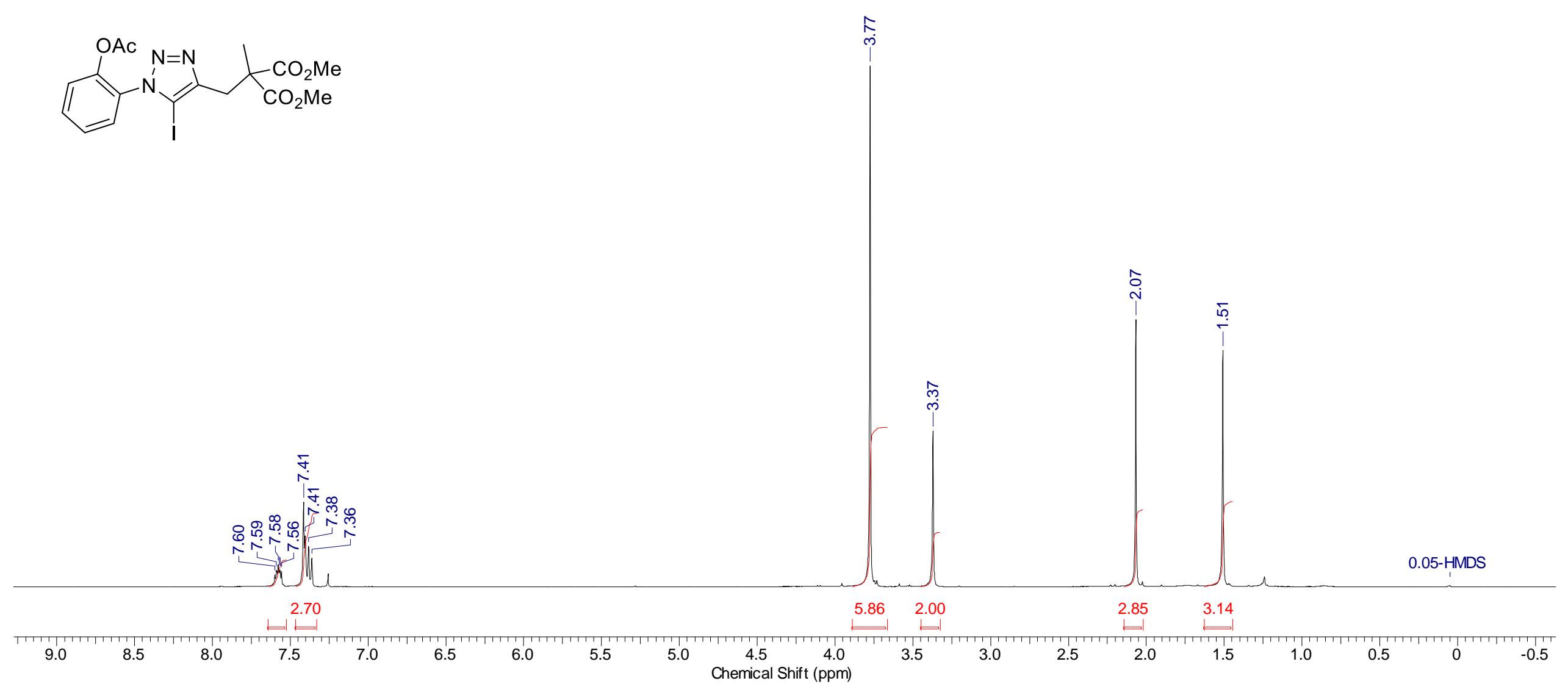




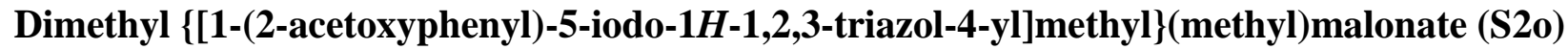

${ }^{13} \mathrm{C}$ NMR (100.6 MHz, $\mathrm{CDCl}_{3}$ )
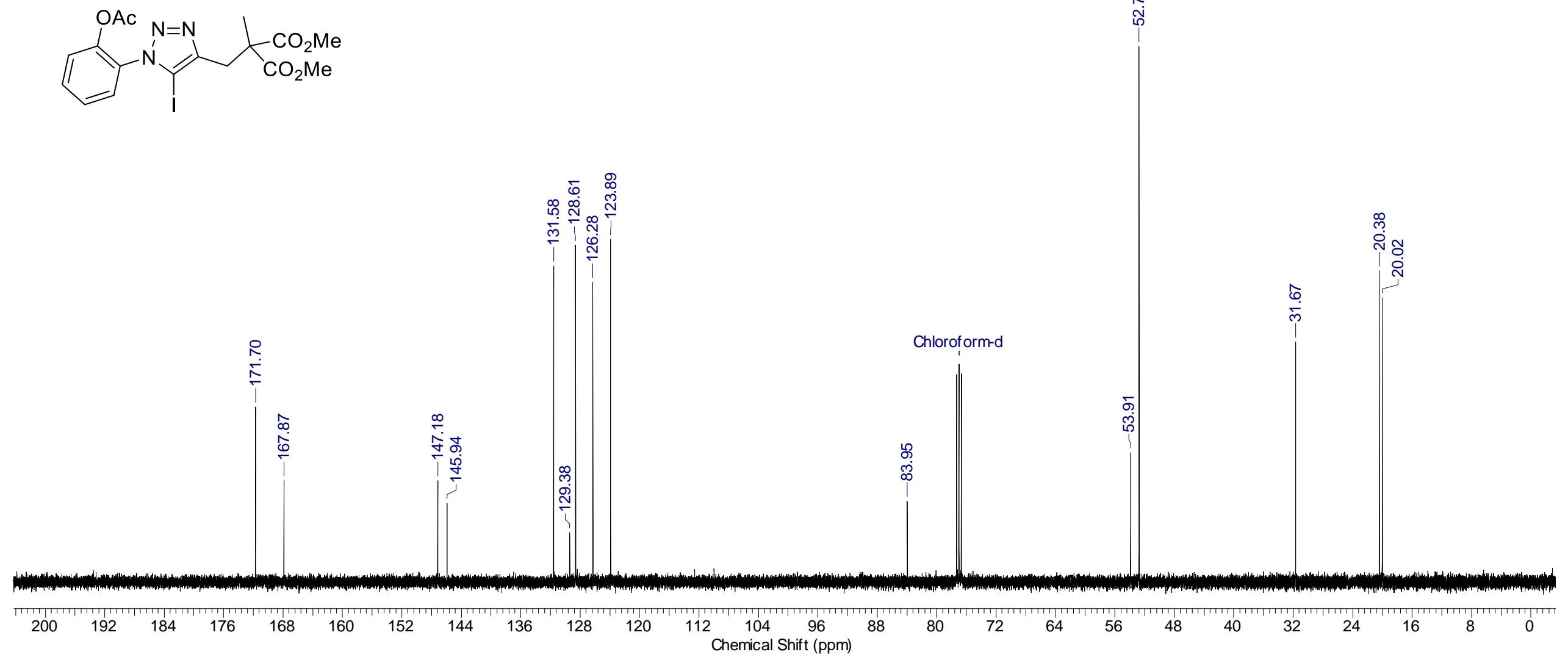


\section{2-(4-Phenyl-5-iodo-1H-1,2,3-triazol-1-yl)phenyl acetate (S2q)}

${ }^{1} \mathrm{H}$ NMR (400 MHz, $\left.\mathrm{CDCl}_{3}\right)$

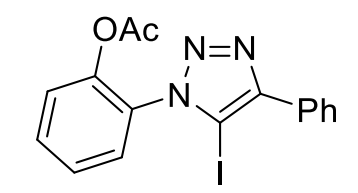

$$
\text { à }
$$

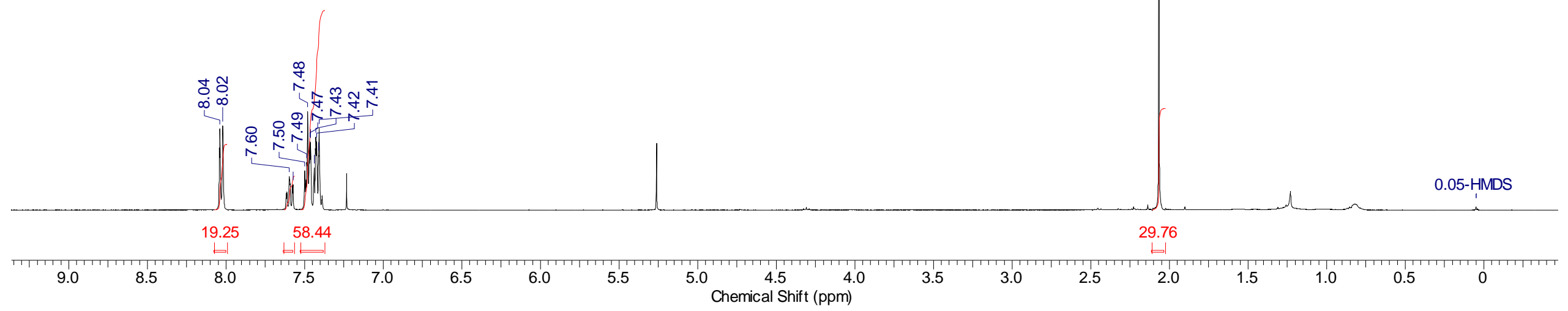




\section{2-(4-Phenyl-5-iodo-1H-1,2,3-triazol-1-yl)phenyl acetate (S2q)}

${ }^{13} \mathrm{C}$ NMR (100.6 MHz, $\mathrm{CDCl}_{3}$ )
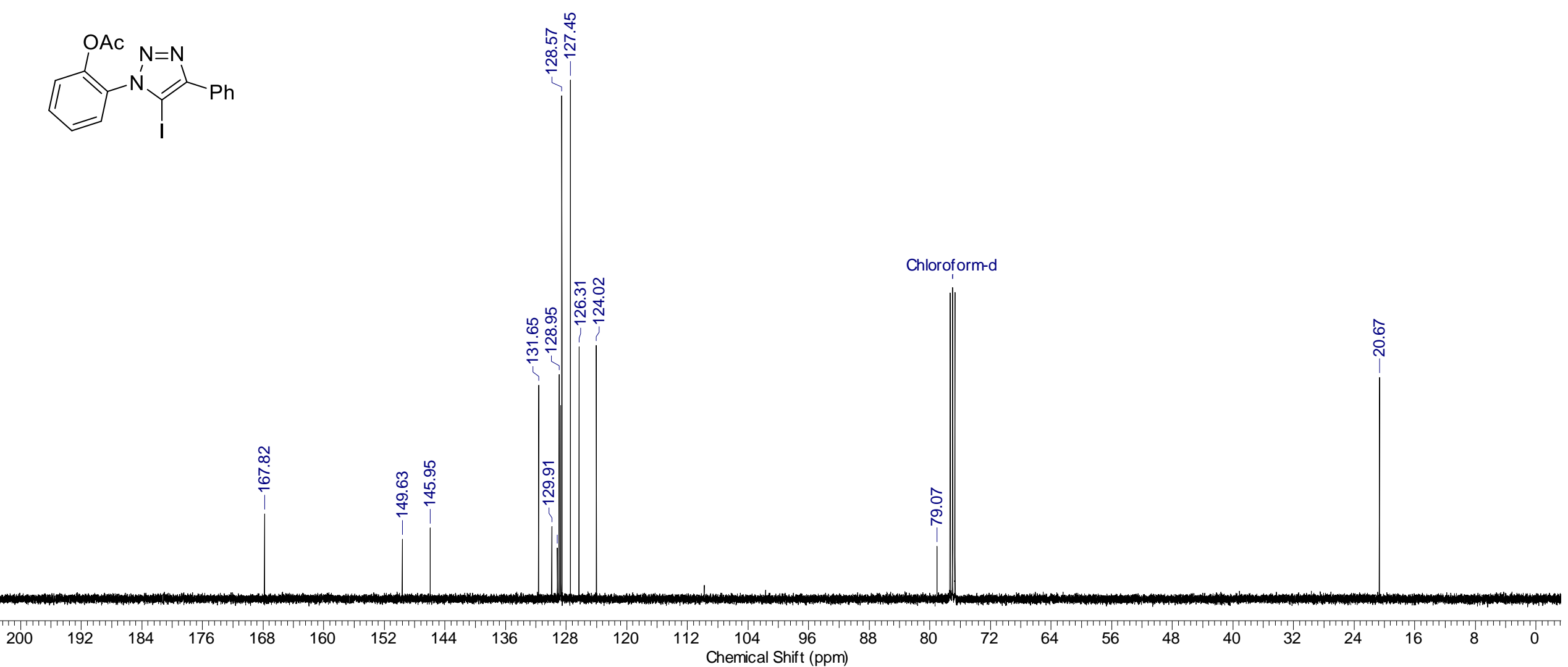


\section{2-[5-Iodo-4-(3-methylbutyl)-1H-1,2,3-triazol-1-yl]phenol (1h)}

${ }^{1} \mathrm{H}$ NMR $\left(600 \mathrm{MHz}, \mathrm{CDCl}_{3}-\mathrm{CD}_{3} \mathrm{OD}\right)$
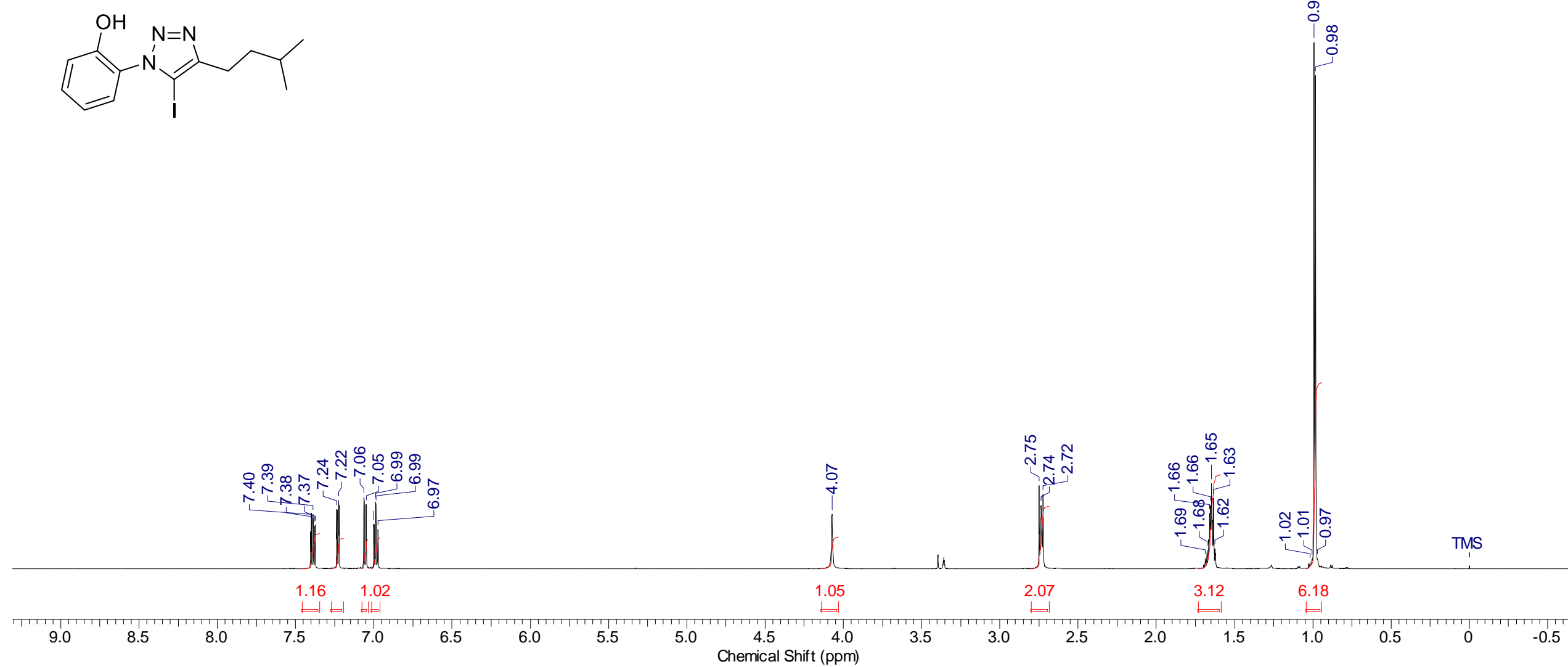
${ }^{13} \mathrm{C}$ NMR (151 MHz, $\left.\mathrm{CDCl}_{3}-\mathrm{CD}_{3} \mathrm{OD}\right)$
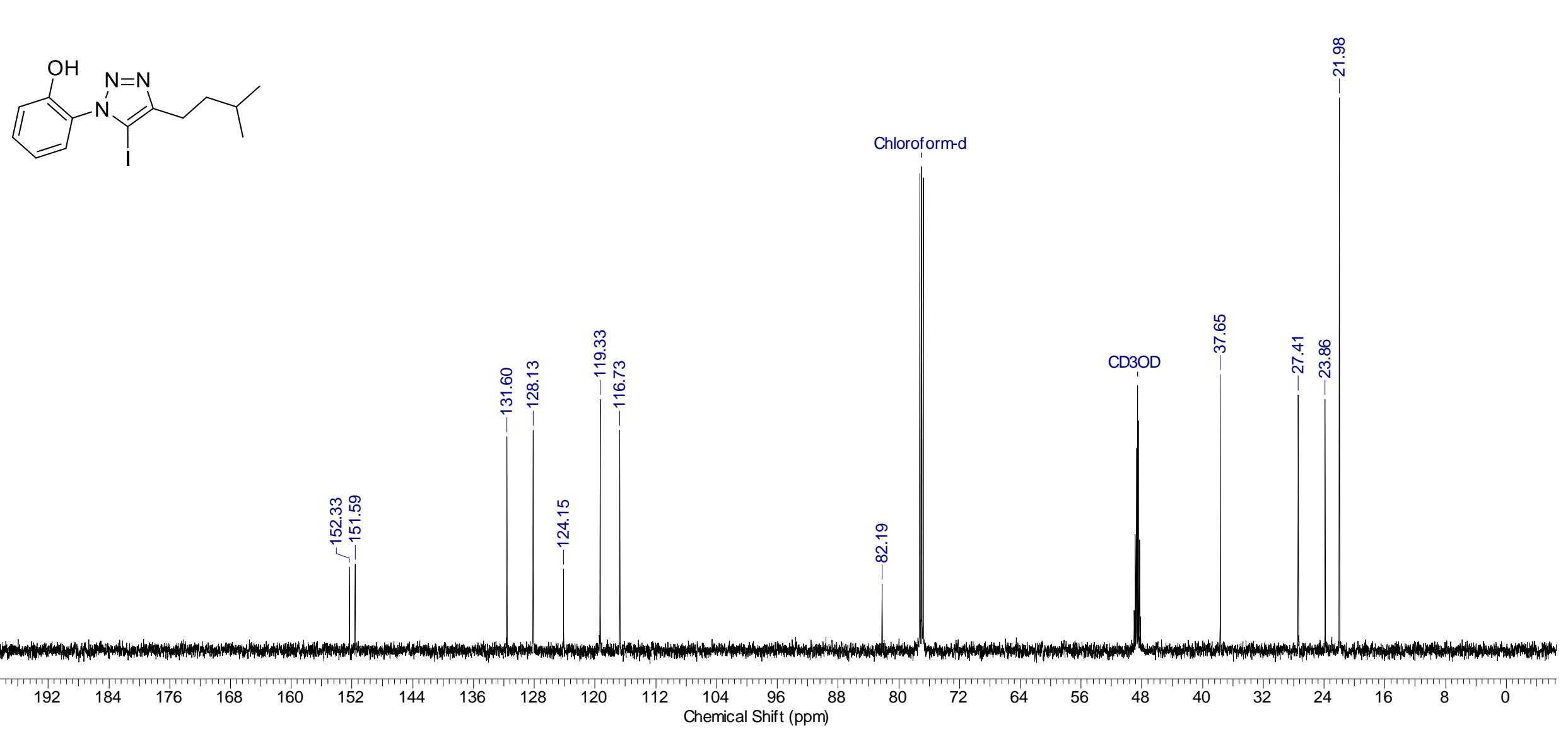


\section{2-(4-Cyclohexyl-5-iodo-1H-1,2,3-triazol-1-yl)phenol (1j)}

${ }^{1} \mathrm{H}$ NMR (600 MHz, $\left.\mathrm{CDCl}_{3}-\mathrm{CD}_{3} \mathrm{OD}\right)$

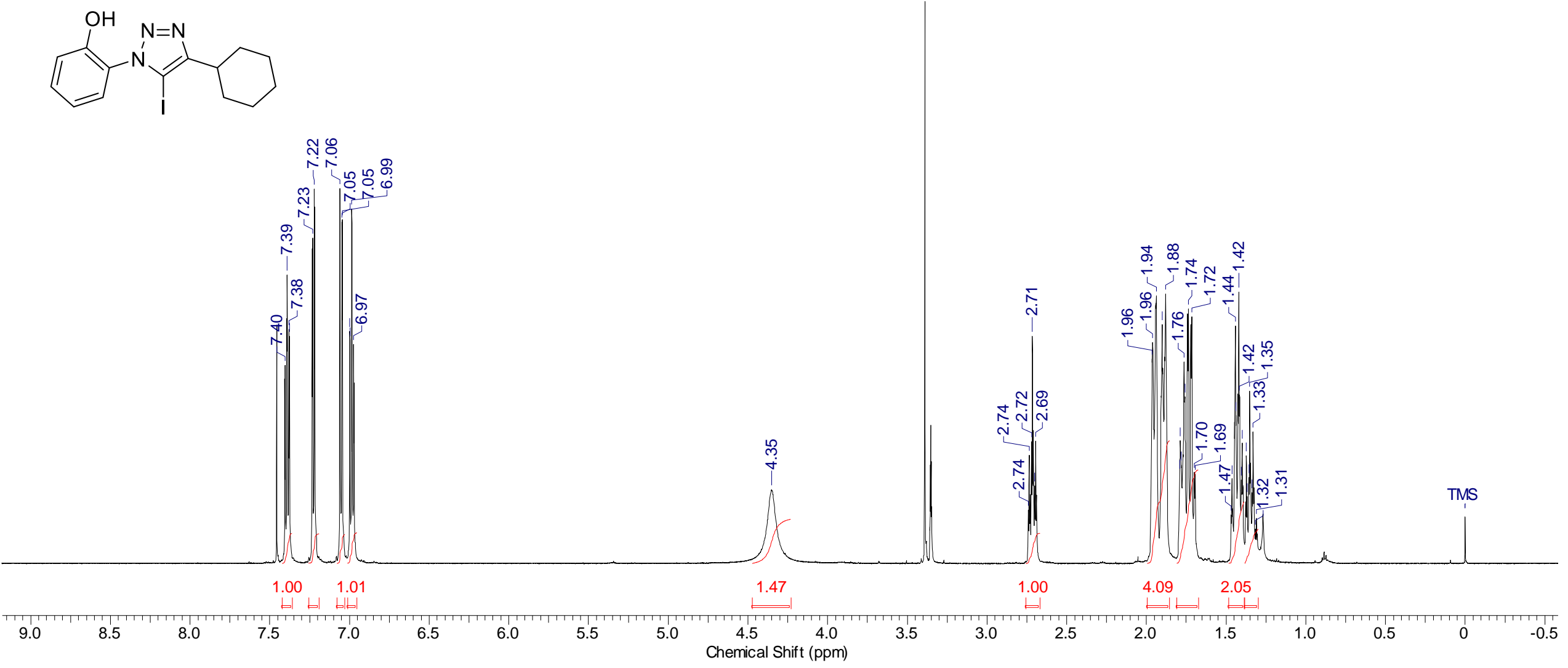


${ }^{13} \mathrm{C}$ NMR (151 MHz, $\left.\mathrm{CDCl}_{3}-\mathrm{CD}_{3} \mathrm{OD}\right)$
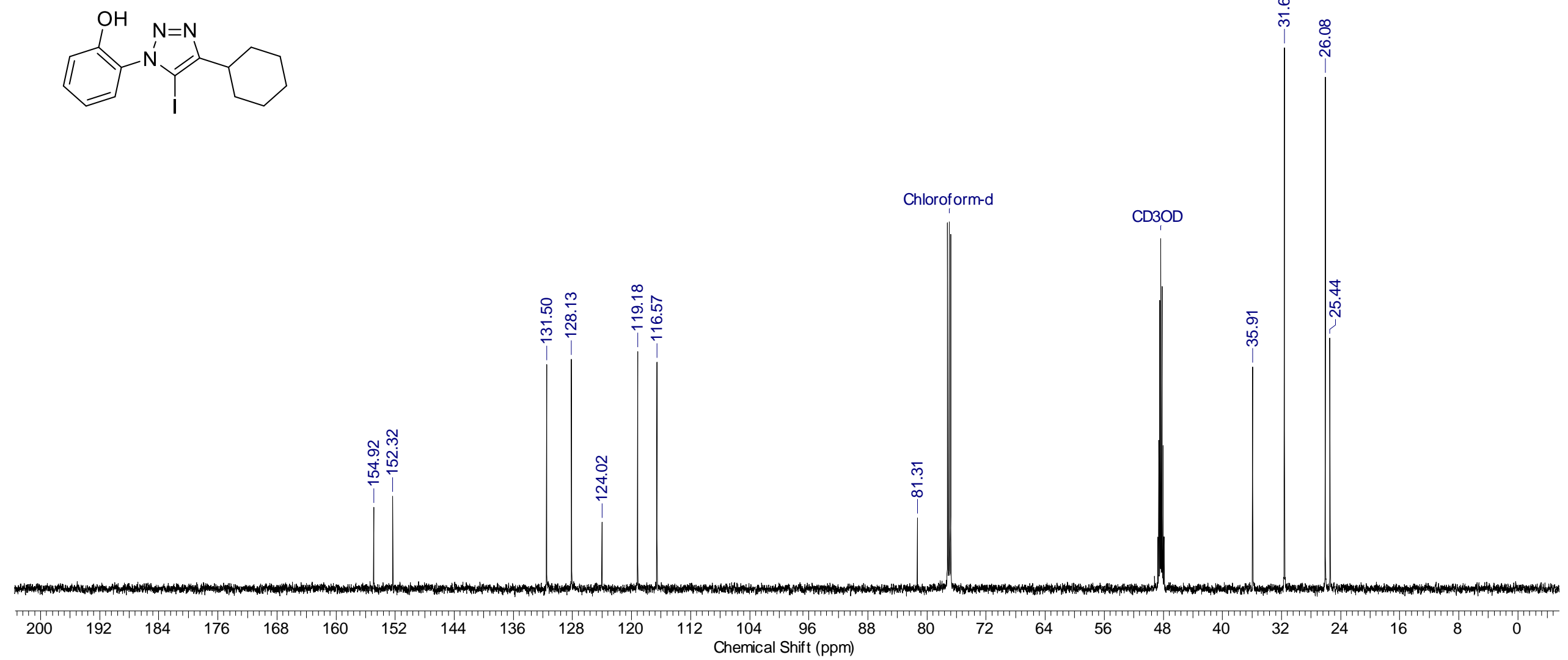
2-[4-(Cyclohexylmethyl)-5-iodo-1H-1,2,3-triazol-1-yl]phenol (1k)

${ }^{1} \mathrm{H}$ NMR $\left(400 \mathrm{MHz}, \mathrm{CDCl}_{3}-\mathrm{CD}_{3} \mathrm{OD}\right)$
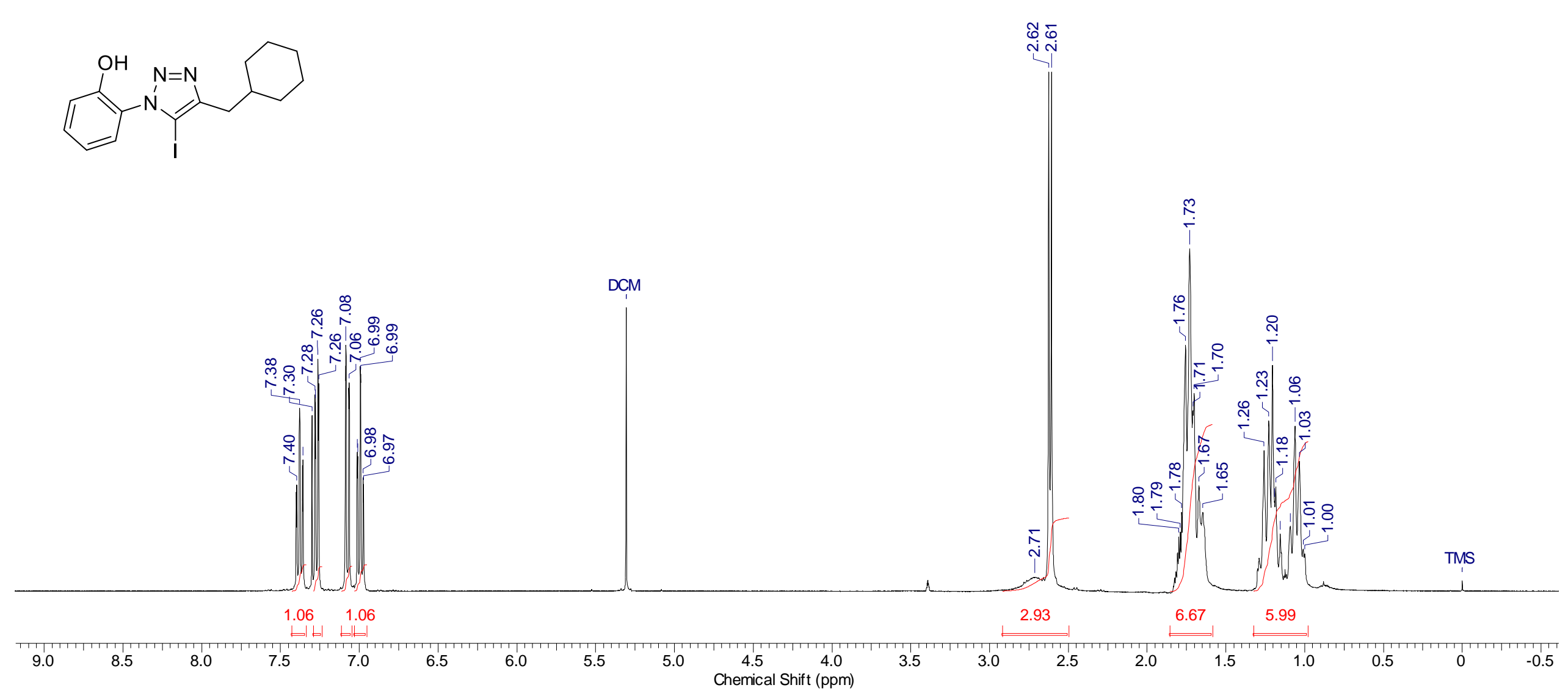
2-[4-(Cyclohexylmethyl)-5-iodo-1H-1,2,3-triazol-1-yl]phenol (1k)

${ }^{13} \mathrm{C}$ NMR (100.6 MHz, $\left.\mathrm{CDCl}_{3}-\mathrm{CD}_{3} \mathrm{OD}\right)$
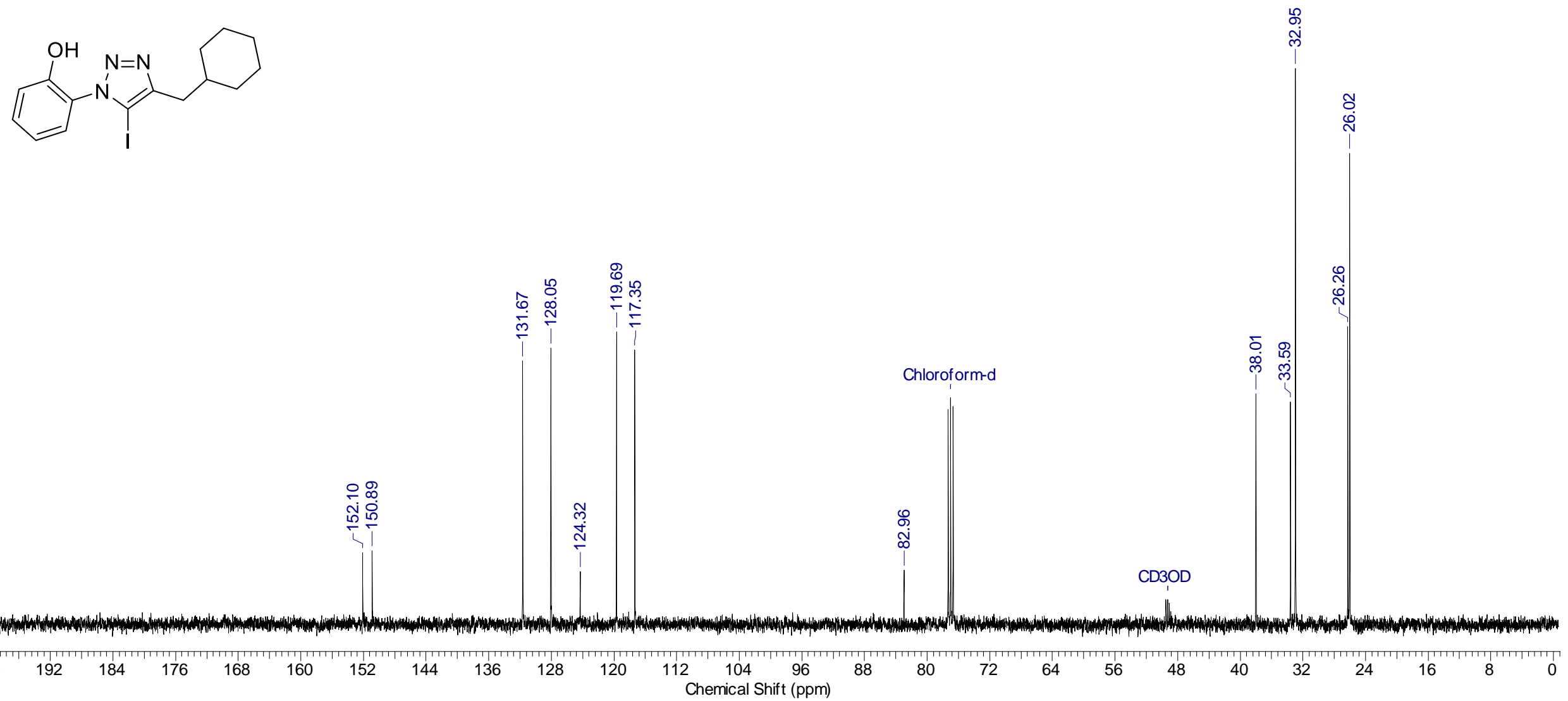
${ }^{1} \mathrm{H}$ NMR (600 MHz, $\left.\mathrm{CDCl}_{3}-\mathrm{CD}_{3} \mathrm{OD}\right)$
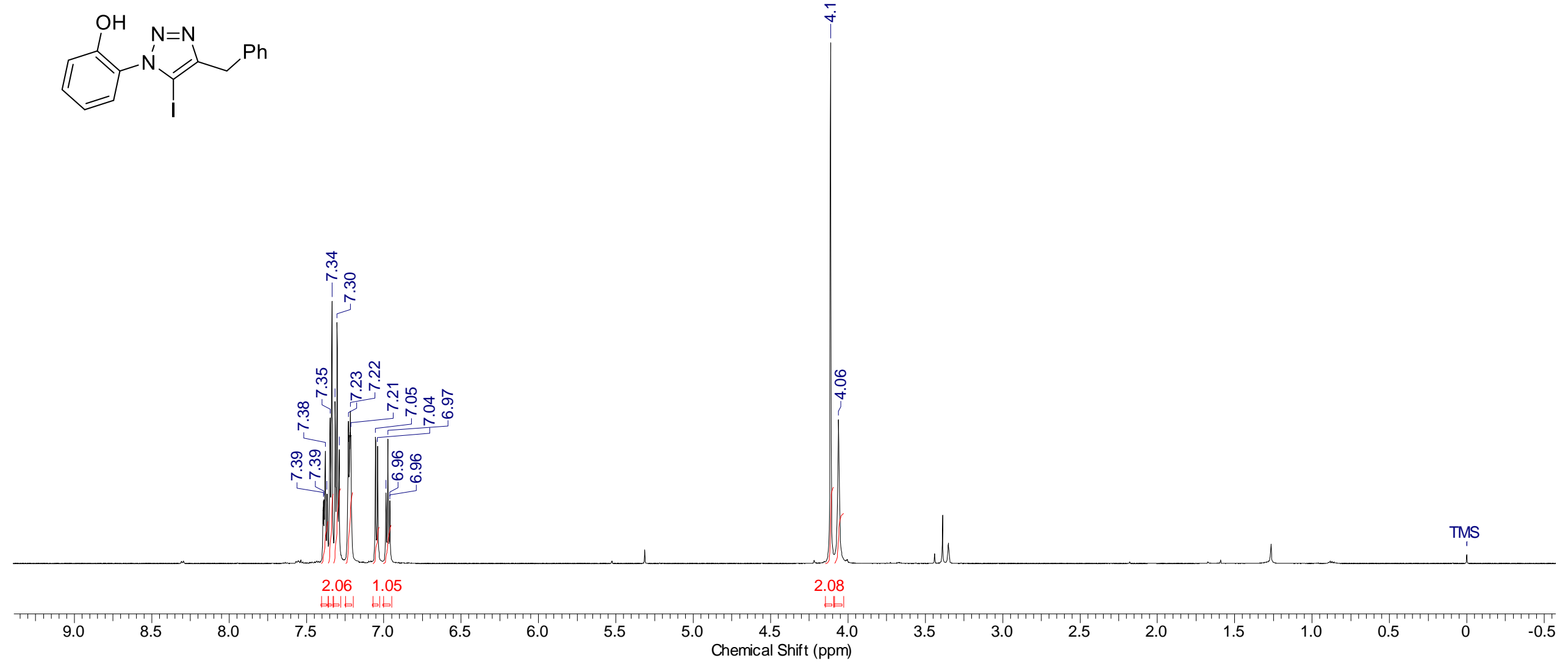
2-(4-Benzyl-5-iodo-1H-1,2,3-triazol-1-yl)phenol (11)

${ }^{13} \mathrm{C}$ NMR (151 MHz, $\left.\mathrm{CDCl}_{3}-\mathrm{CD}_{3} \mathrm{OD}\right)$
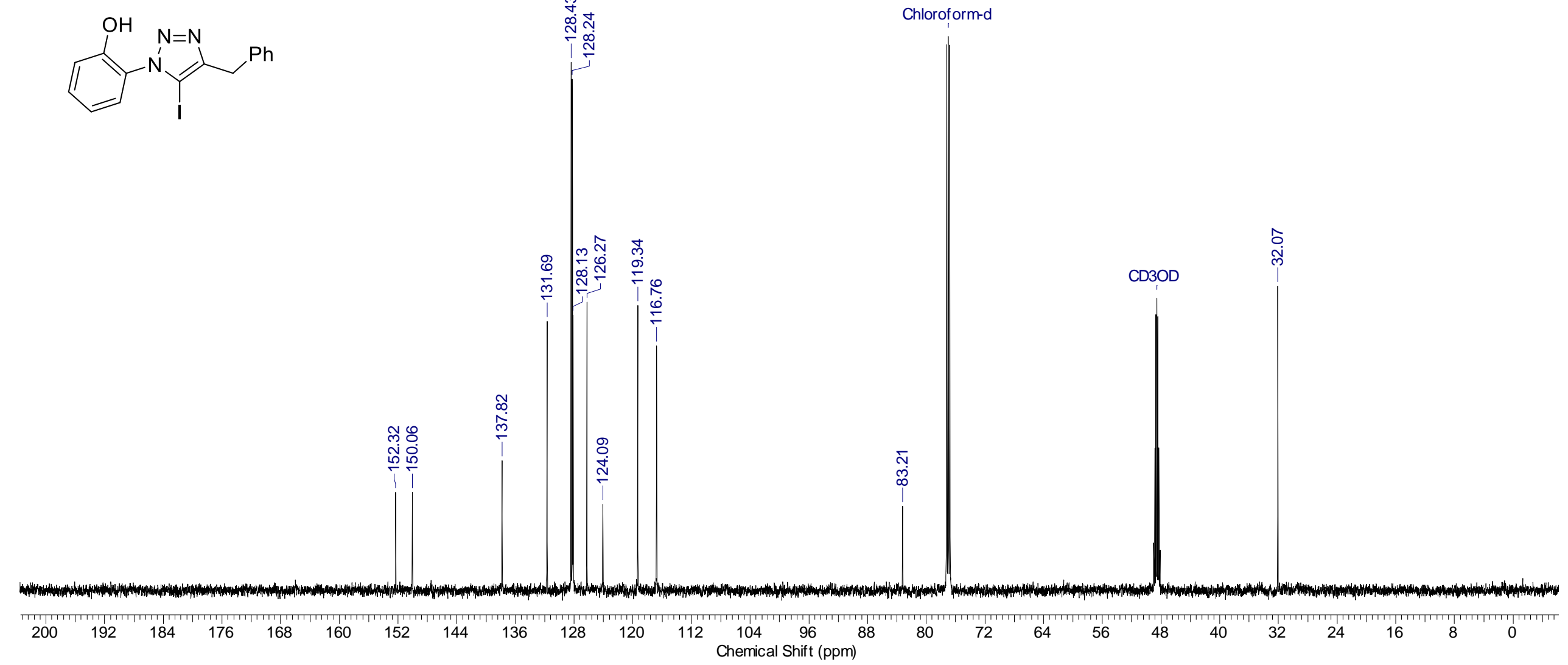


\section{$N$-\{[1-(2-Hydroxyphenyl)-5-iodo-1H-1,2,3-triazol-4-yl]methyl\}acetamide (1n)}

${ }^{1} \mathrm{H}$ NMR (400 MHz, $\left.\mathrm{CDCl}_{3}-\mathrm{CD}_{3} \mathrm{OD}\right)$

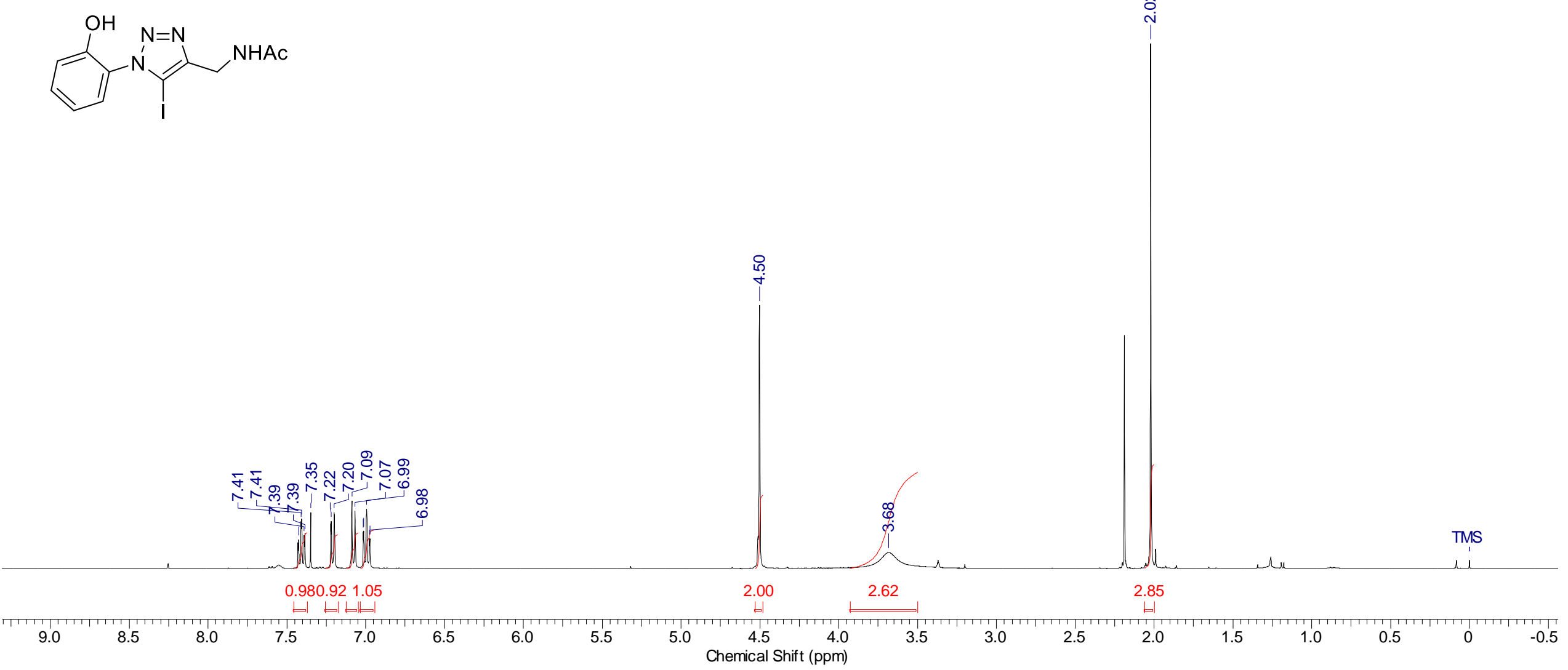


$N$-\{[1-(2-Hydroxyphenyl)-5-iodo-1H-1,2,3-triazol-4-yl]methyl $\}$ acetamide (1n)

${ }^{13} \mathrm{C}$ NMR (100.6 MHz, $\left.\mathrm{CDCl}_{3}-\mathrm{CD}_{3} \mathrm{OD}\right)$

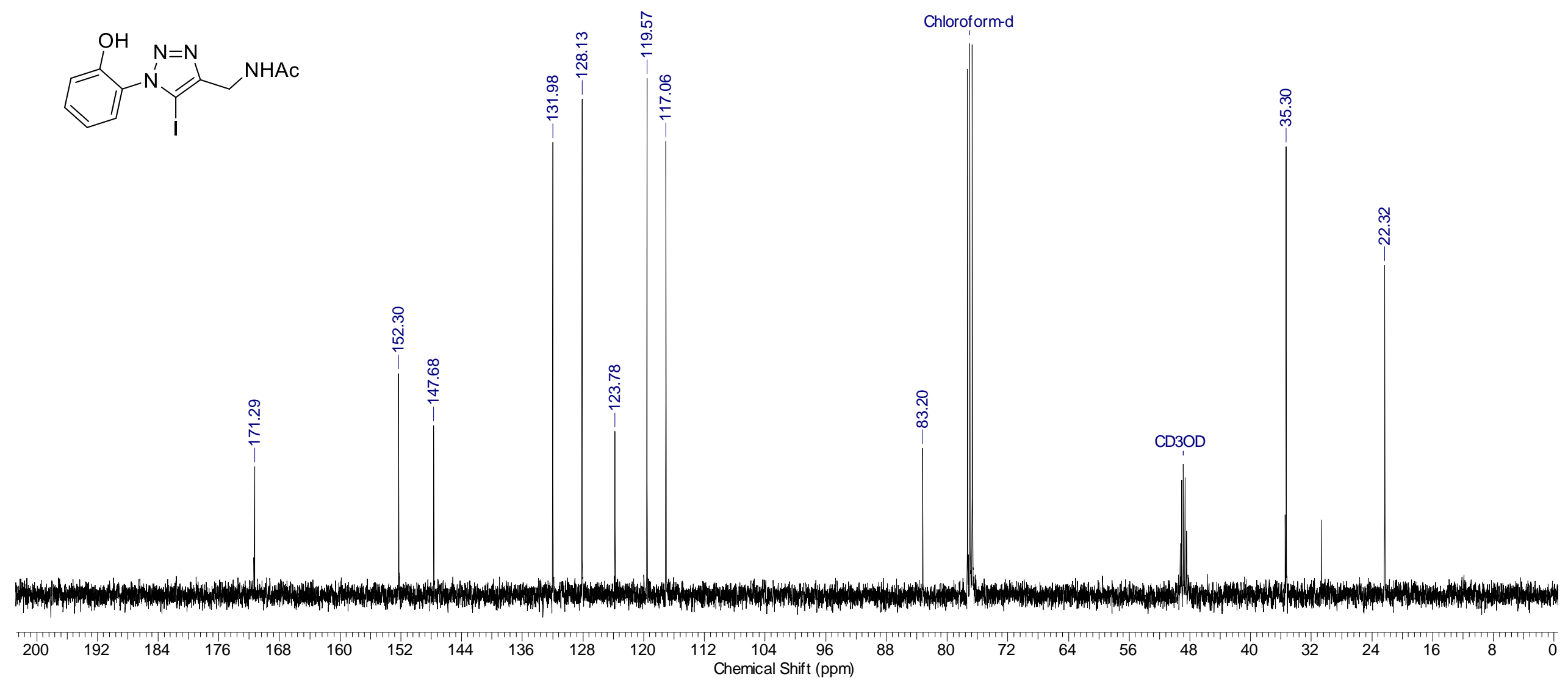


Dimethyl $\{[1-(2-h y d r o x y p h e n y l)-5-i o d o-1 H-1,2,3-t r i a z o l-4-y l] m e t h y l\}$ (methyl)malonate (10)

${ }^{1} \mathrm{H}$ NMR $\left(400 \mathrm{MHz}, \mathrm{CDCl}_{3}-\mathrm{CD}_{3} \mathrm{OD}\right)$

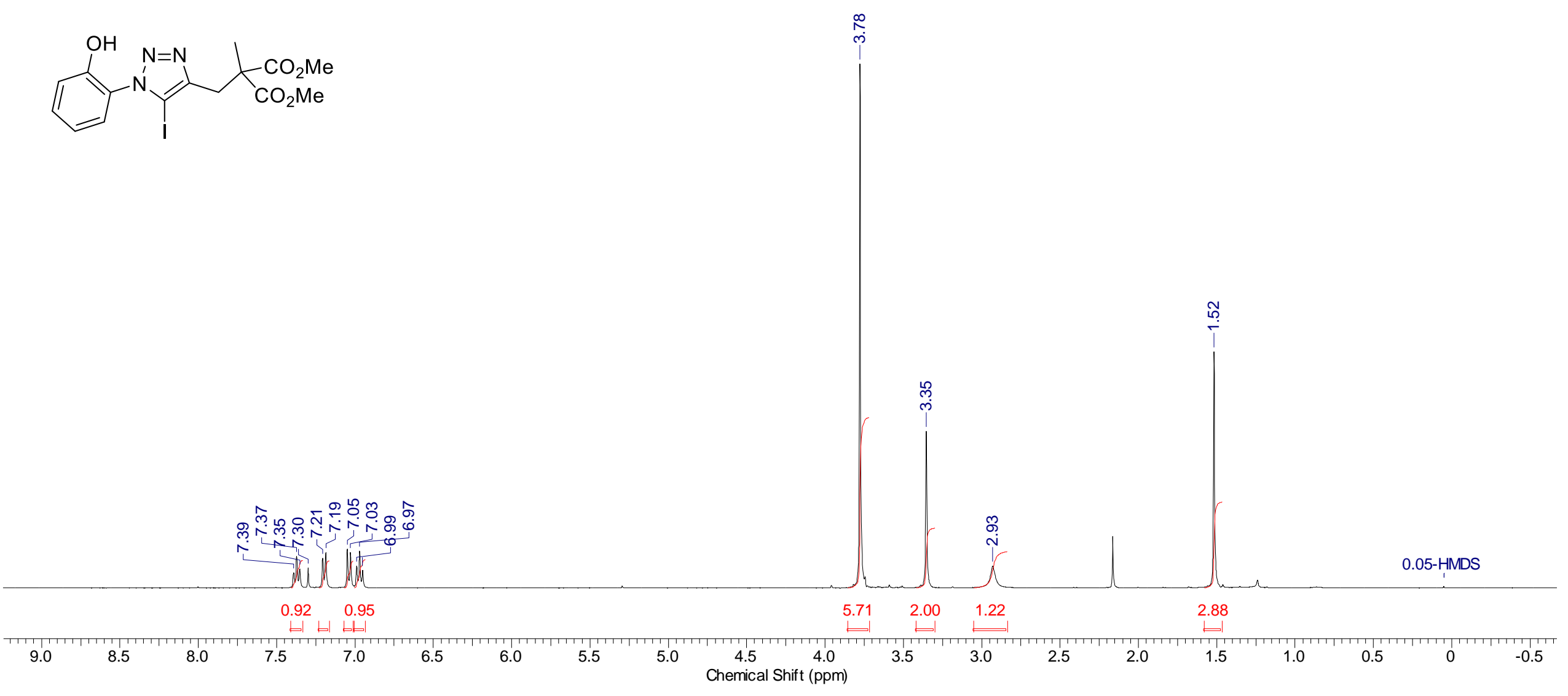




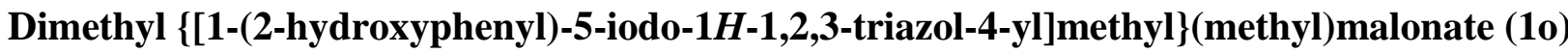

${ }^{13} \mathrm{C}$ NMR (100.6 MHz, $\left.\mathrm{CDCl}_{3}-\mathrm{CD}_{3} \mathrm{OD}\right)$
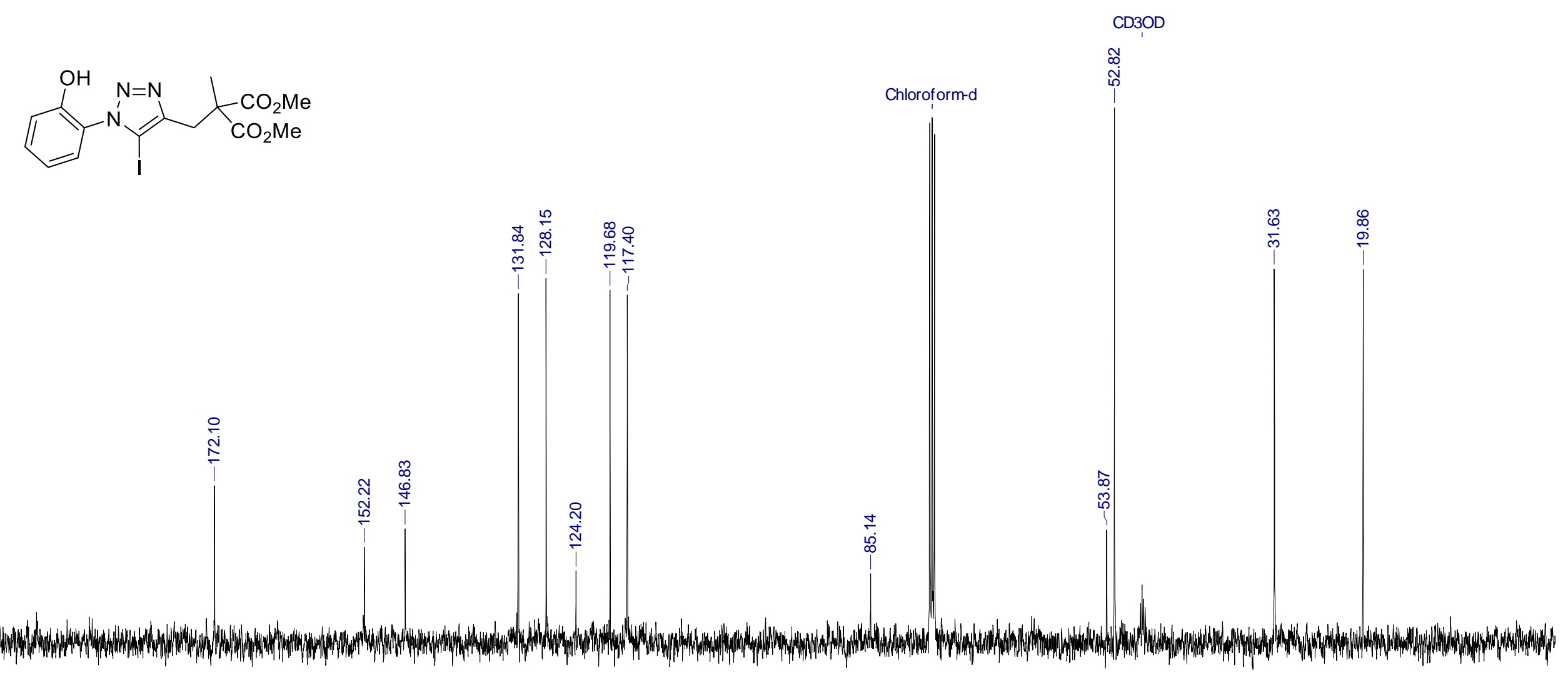

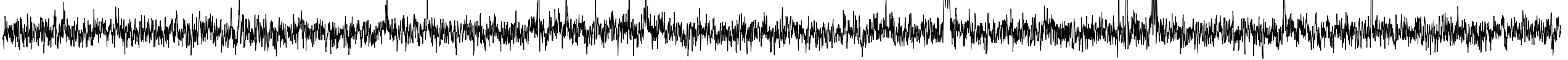

$200192 \quad 184 \begin{array}{lll}176 \\ 208\end{array}$




\section{2-(4-Phenyl-5-iodo-1H-1,2,3-triazol-1-yl)phenol (1q)}

${ }^{1} \mathrm{H}$ NMR (400 MHz, DMSO-d ${ }^{6}$ )
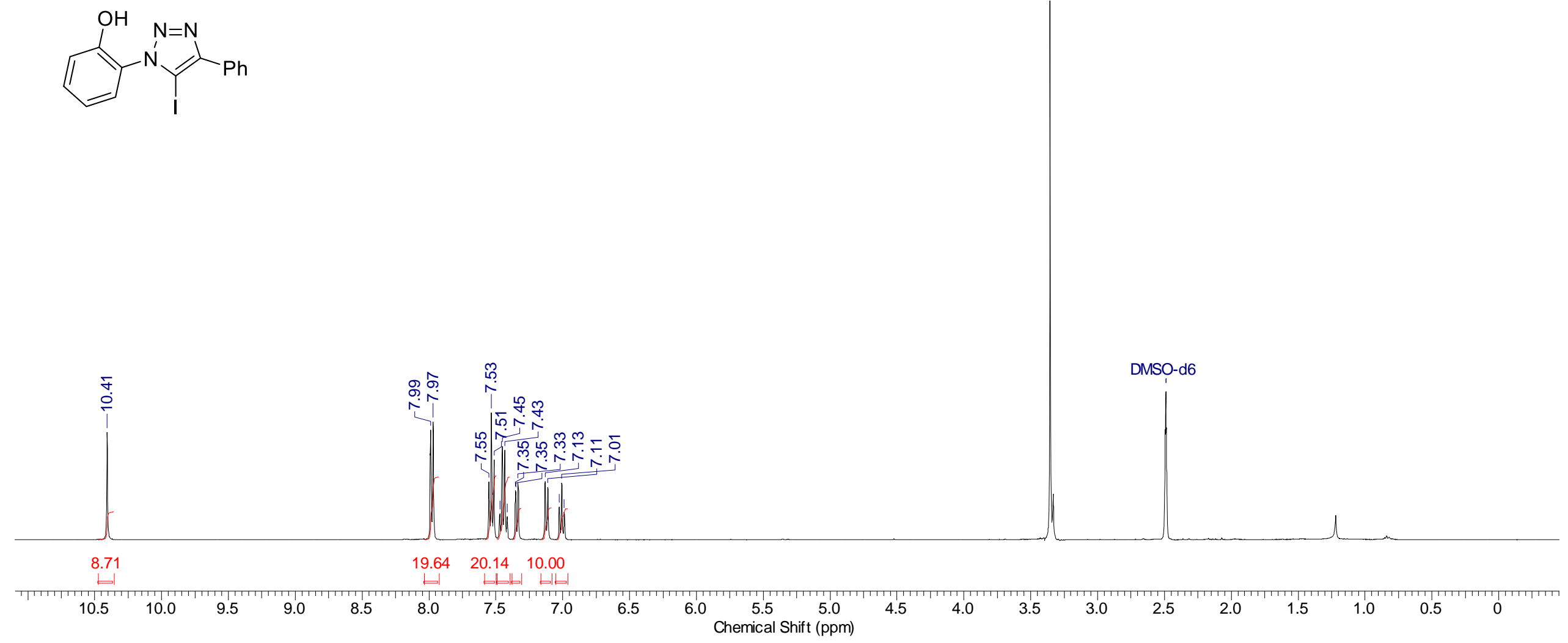
${ }^{13} \mathrm{C}$ NMR (100.6 MHz, DMSO-d $\left.\mathrm{d}^{6}\right)$
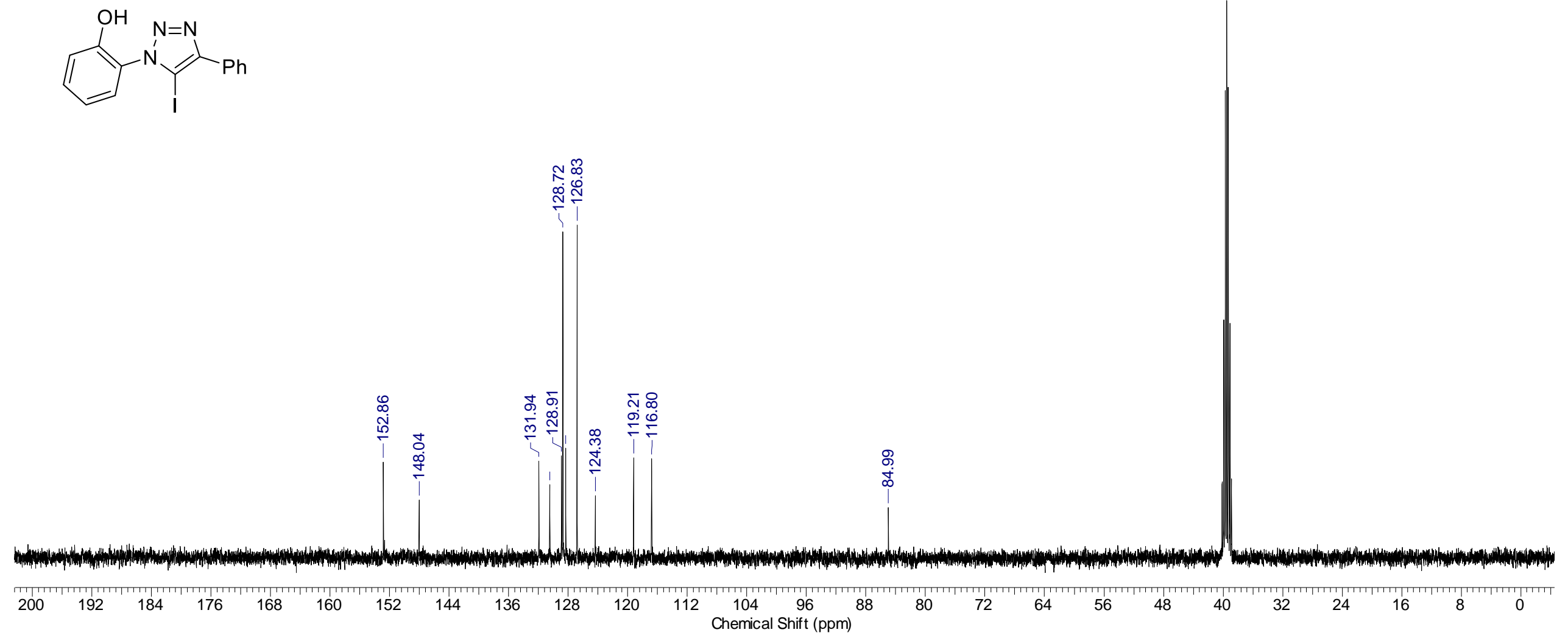
2-[1-(1,3-Benzothiazol-2-ylthio)pentyl]-1,3-benzoxazole (2a)

${ }^{1} \mathrm{H}$ NMR $\left(400 \mathrm{MHz}, \mathrm{CDCl}_{3}\right)$
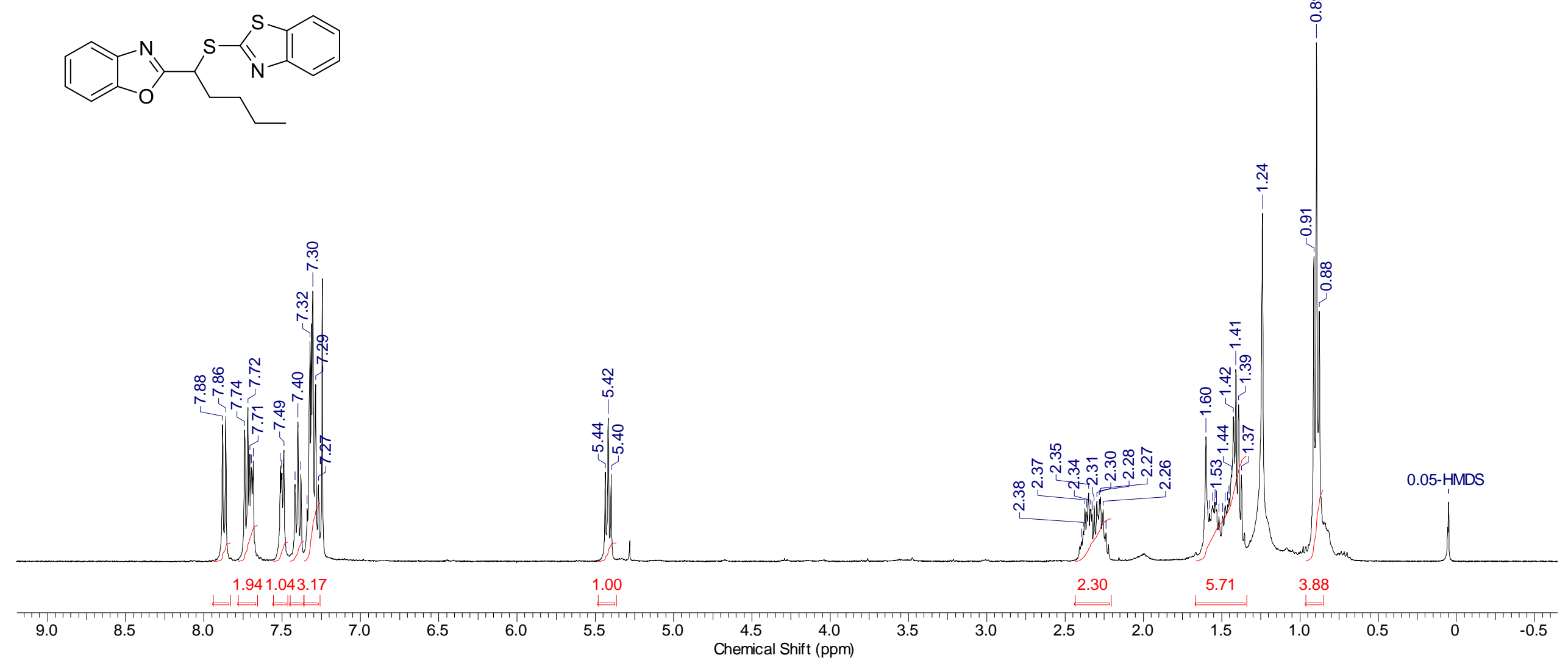
2-[1-(1,3-Benzothiazol-2-ylthio)pentyl]-1,3-benzoxazole (2a)

${ }^{13} \mathrm{C}$ NMR (100.6 MHz, $\mathrm{CDCl}_{3}$ )
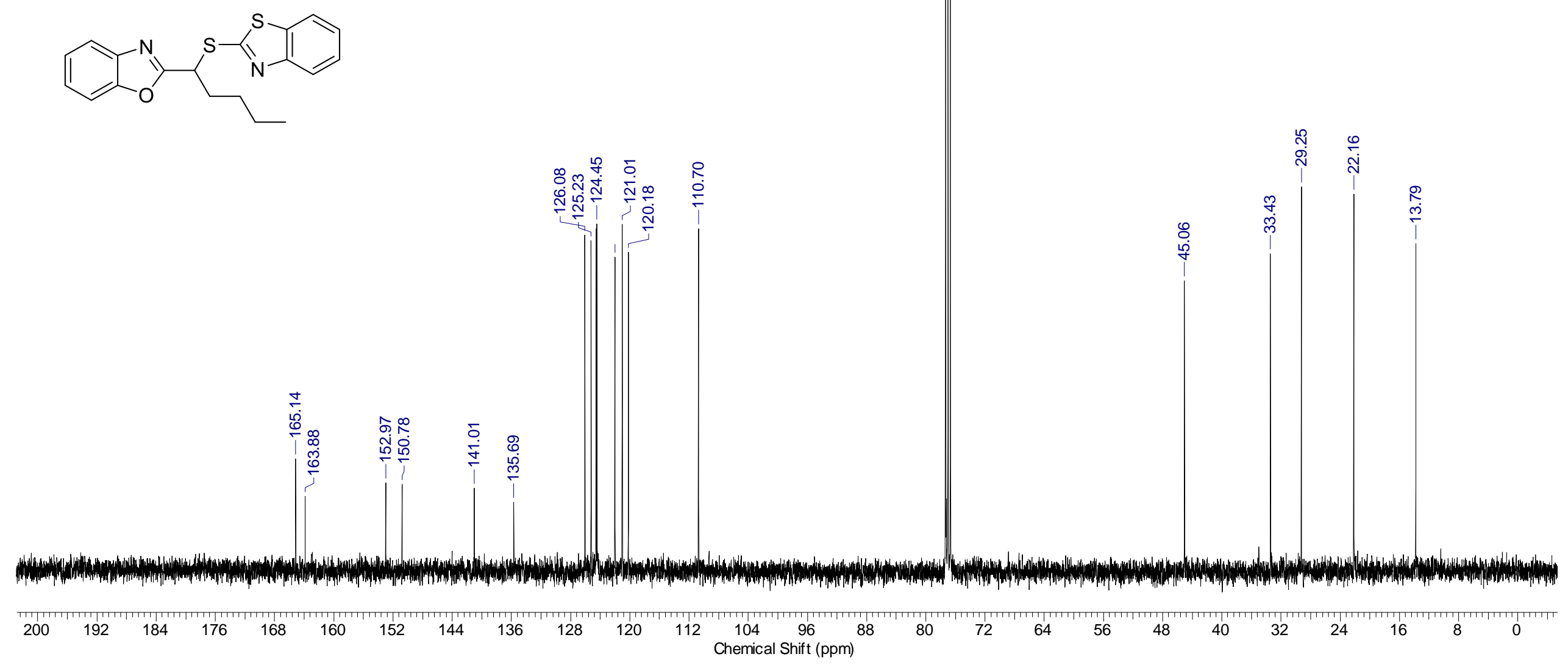
2-\{[1-(1,3-Benzoxazol-2-yl)pentyl]thio\}-1,3-benzoxazole (2b)

${ }^{1} \mathrm{H}$ NMR (400 MHz, $\mathrm{CDCl}_{3}$ )
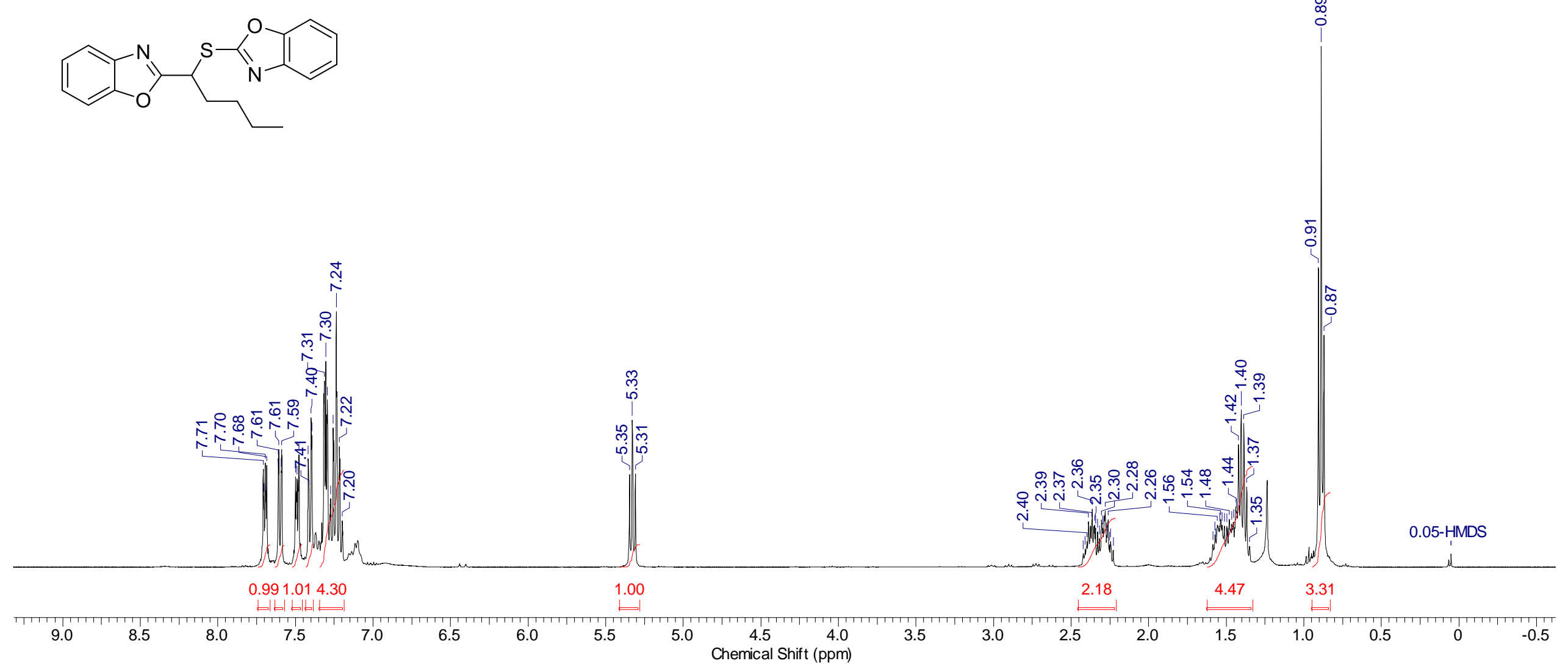


\section{2-\{[1-(1,3-Benzoxazol-2-yl)pentyl]thio\}-1,3-benzoxazole (2b)}

${ }^{13} \mathrm{C}$ NMR (100.6 MHz, $\mathrm{CDCl}_{3}$ )

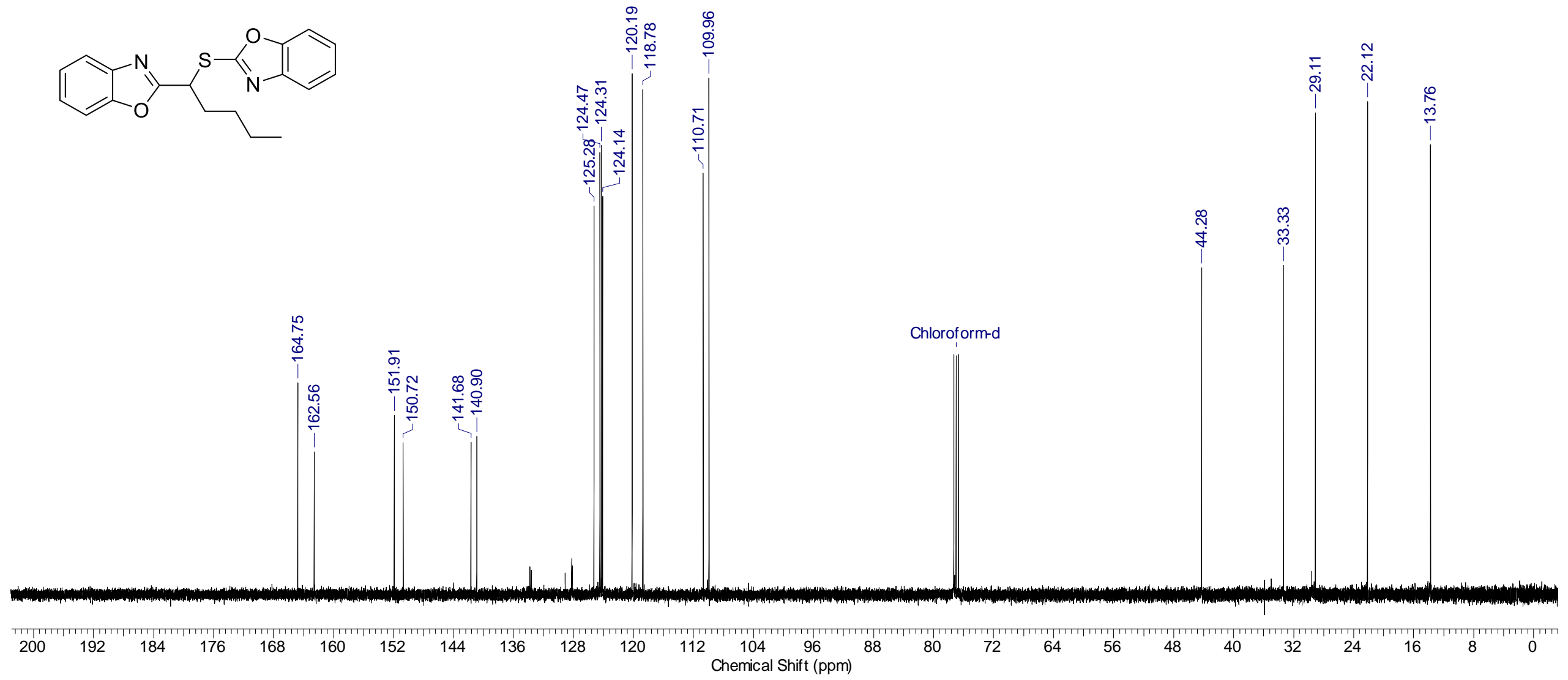




\section{2-[1-(1H-Benzimidazol-2-ylthio)pentyl]-1,3-benzoxazole (2c)}

${ }^{1} \mathrm{H}$ NMR (400 MHz, $\mathrm{CDCl}_{3}$ )
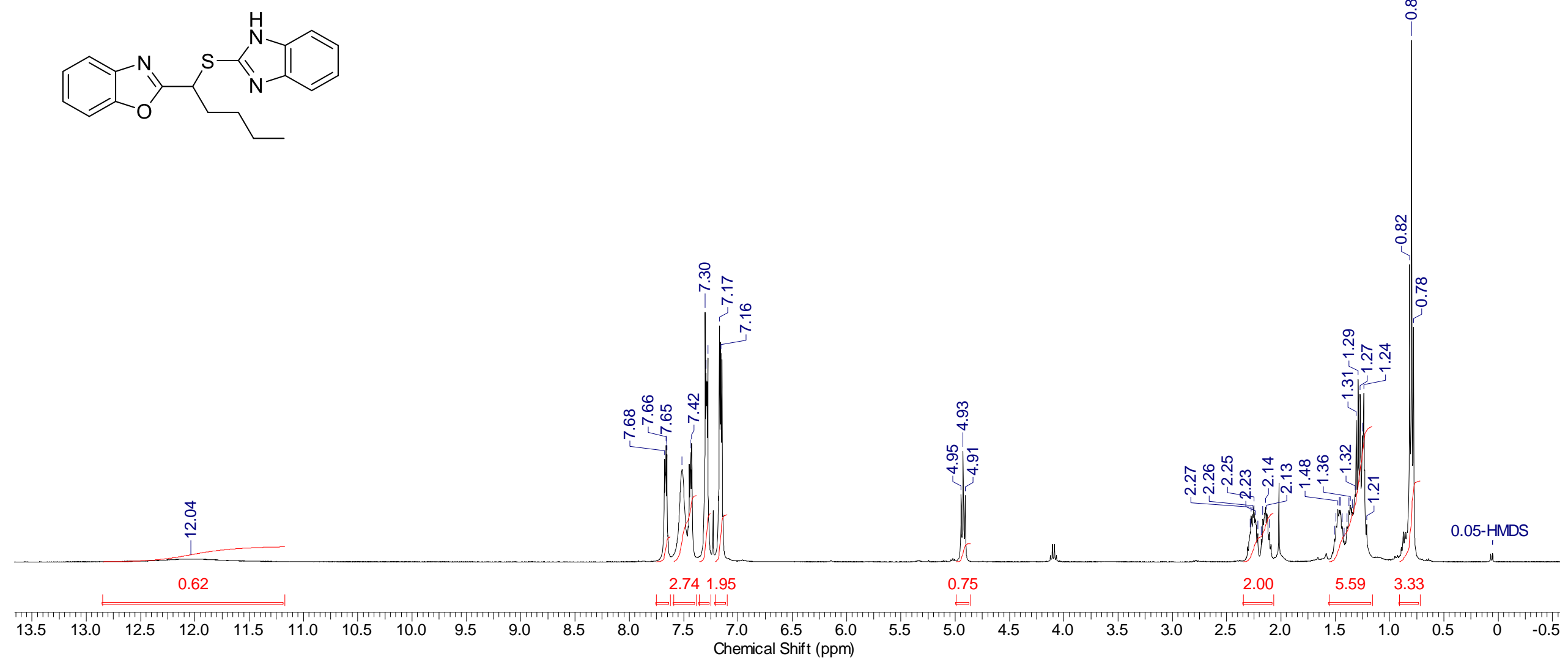


\section{2-[1-(1H-Benzimidazol-2-ylthio)pentyl]-1,3-benzoxazole (2c)}

${ }^{13} \mathrm{C}$ NMR (100.6 MHz, $\mathrm{CDCl}_{3}$ )

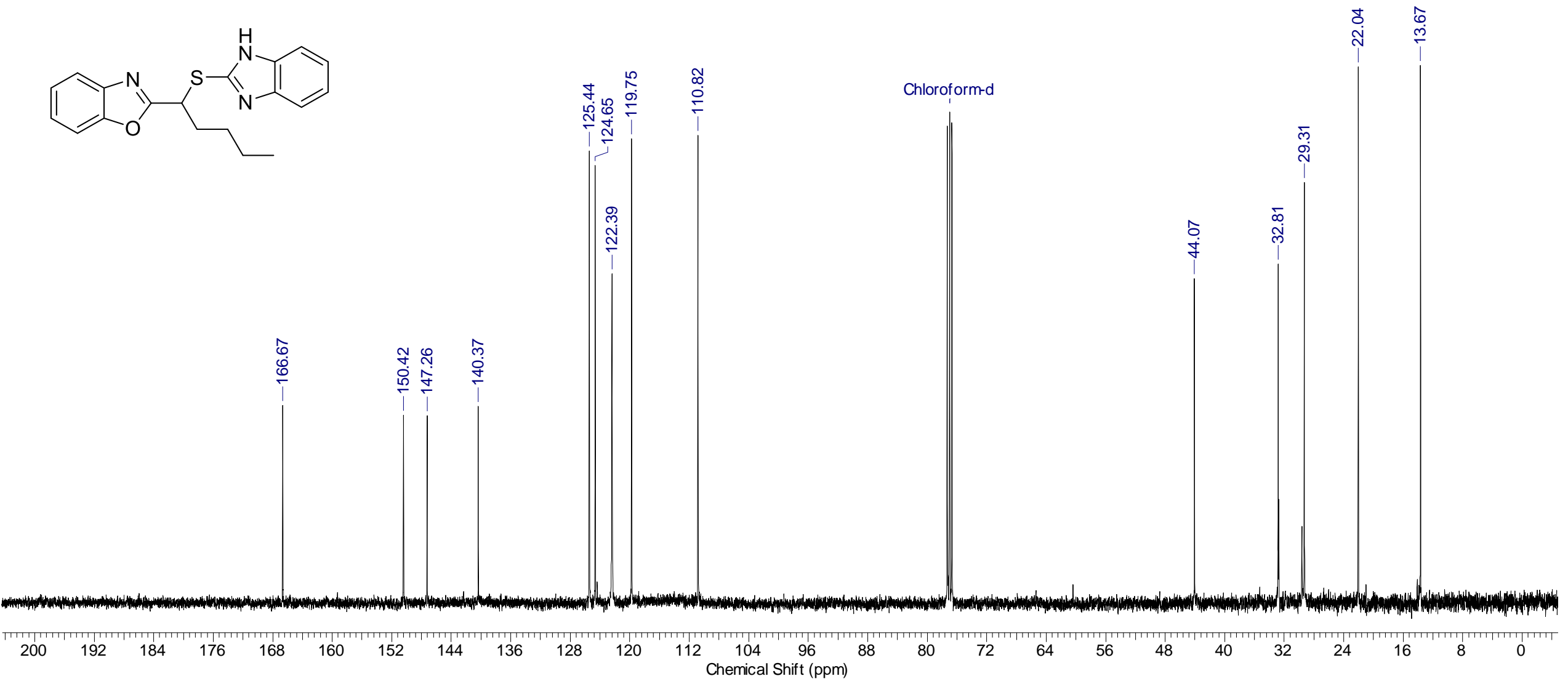


2-[1-(1H-Benzimidazol-2-ylthio)pentyl]-1,3-benzoxazole (2c)

${ }^{1} \mathrm{H}-{ }^{13} \mathrm{C}$ HSQC NMR

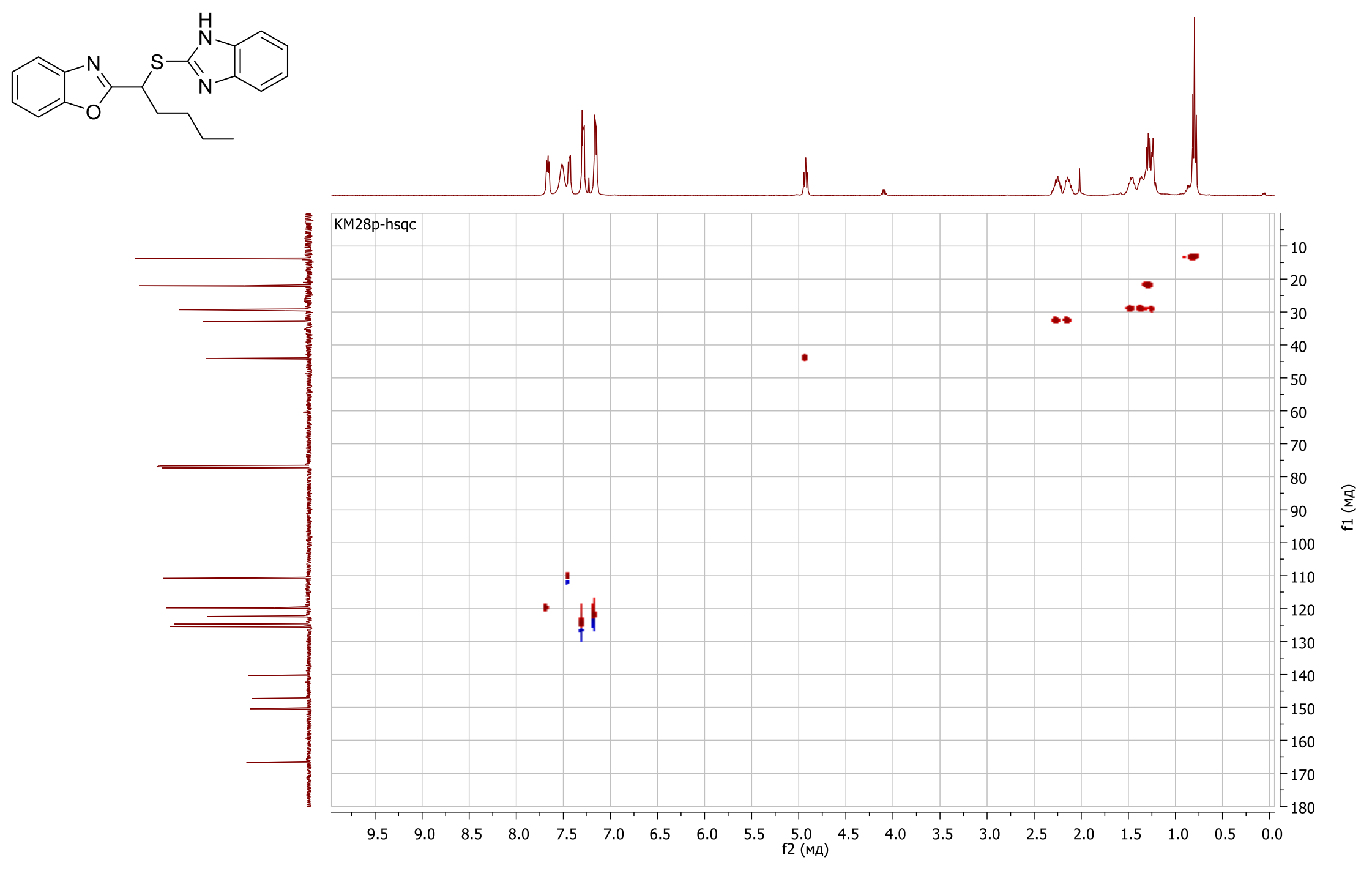




\section{2-[1-(1H-Benzimidazol-2-ylthio)pentyl]-1,3-benzoxazole (2c)}

${ }^{1} \mathrm{H}-{ }^{13} \mathrm{C}$ HMBC NMR

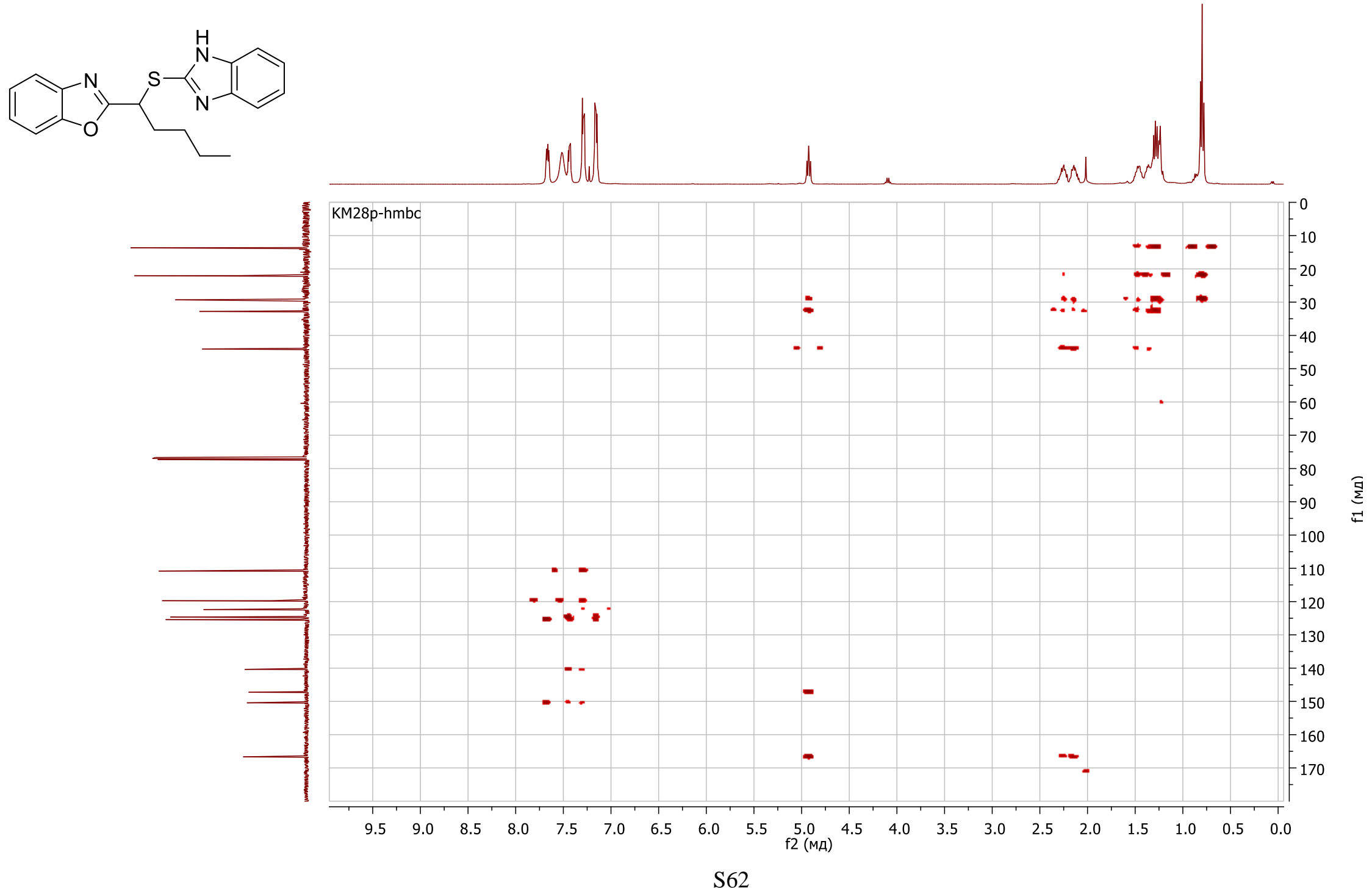


2-\{1-[(5-Methyl-1,3,4-oxadiazol-2-yl)thio]pentyl\}-1,3-benzoxazole (2d)

${ }^{1} \mathrm{H}$ NMR $\left(400 \mathrm{MHz}, \mathrm{CDCl}_{3}\right)$

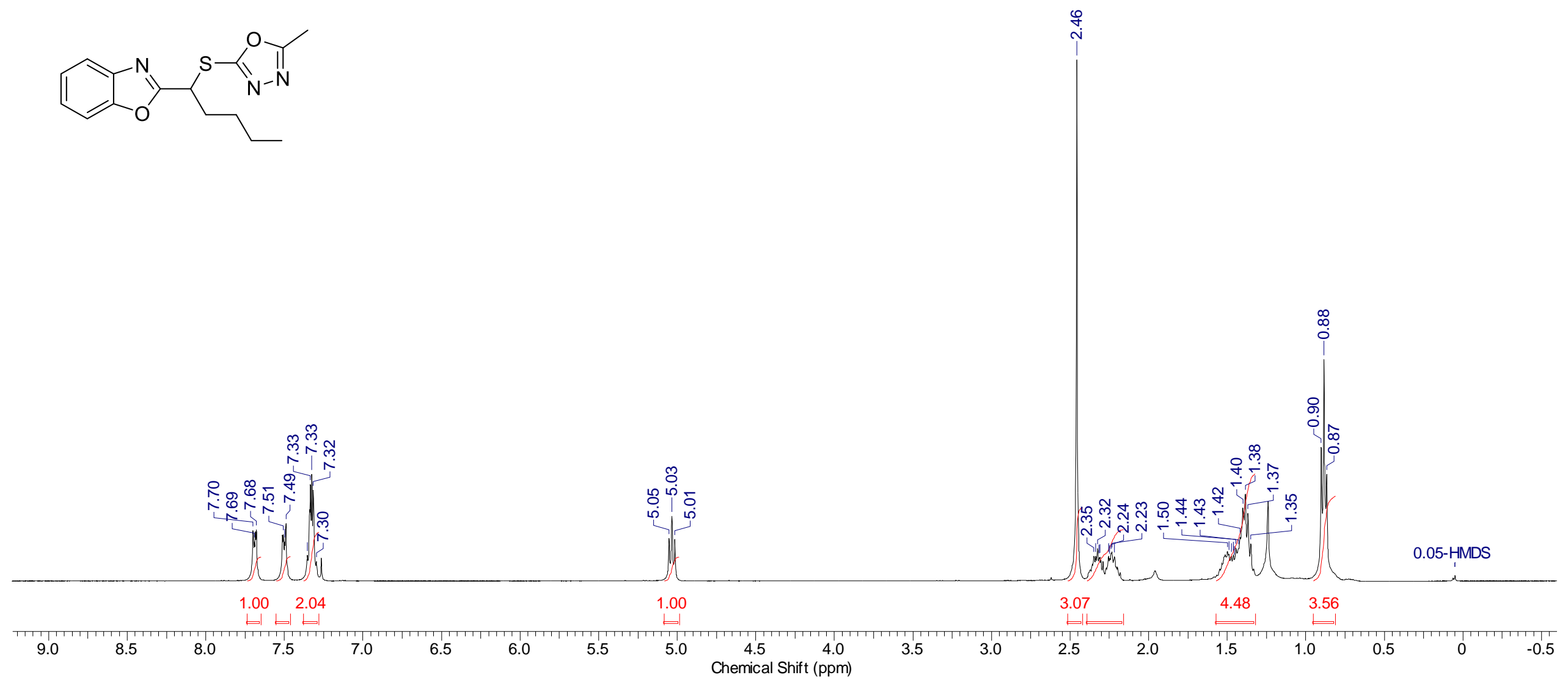


2-\{1-[(5-Methyl-1,3,4-oxadiazol-2-yl)thio]pentyl\}-1,3-benzoxazole (2d)

${ }^{13} \mathrm{C}$ NMR (100.6 MHz, $\mathrm{CDCl}_{3}$ )

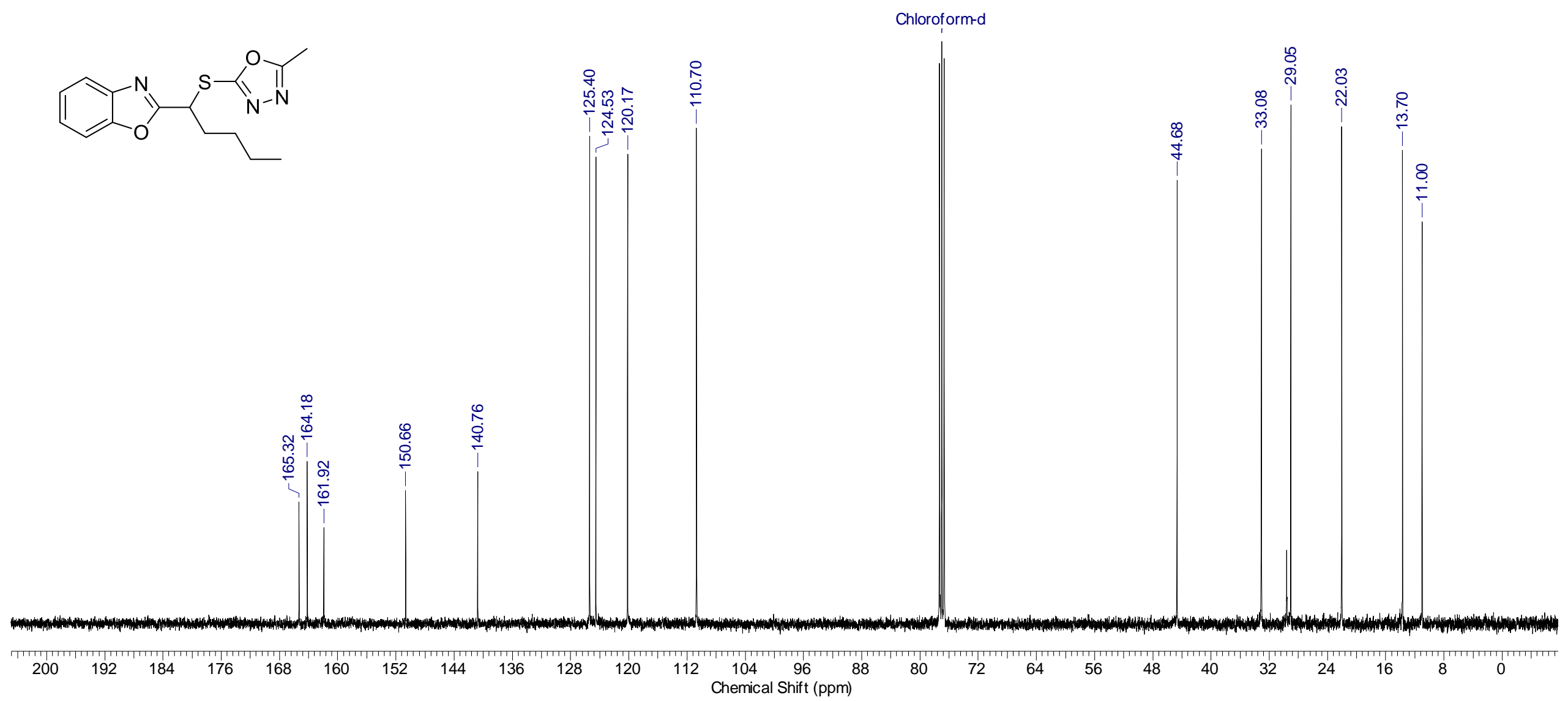


2-\{1-[(5-Methyl-1,3,4-thiadiazol-2-yl)thio]pentyl\}-1,3-benzoxazole (2e)

${ }^{1} \mathrm{H}$ NMR (400 MHz, $\mathrm{CDCl}_{3}$ )

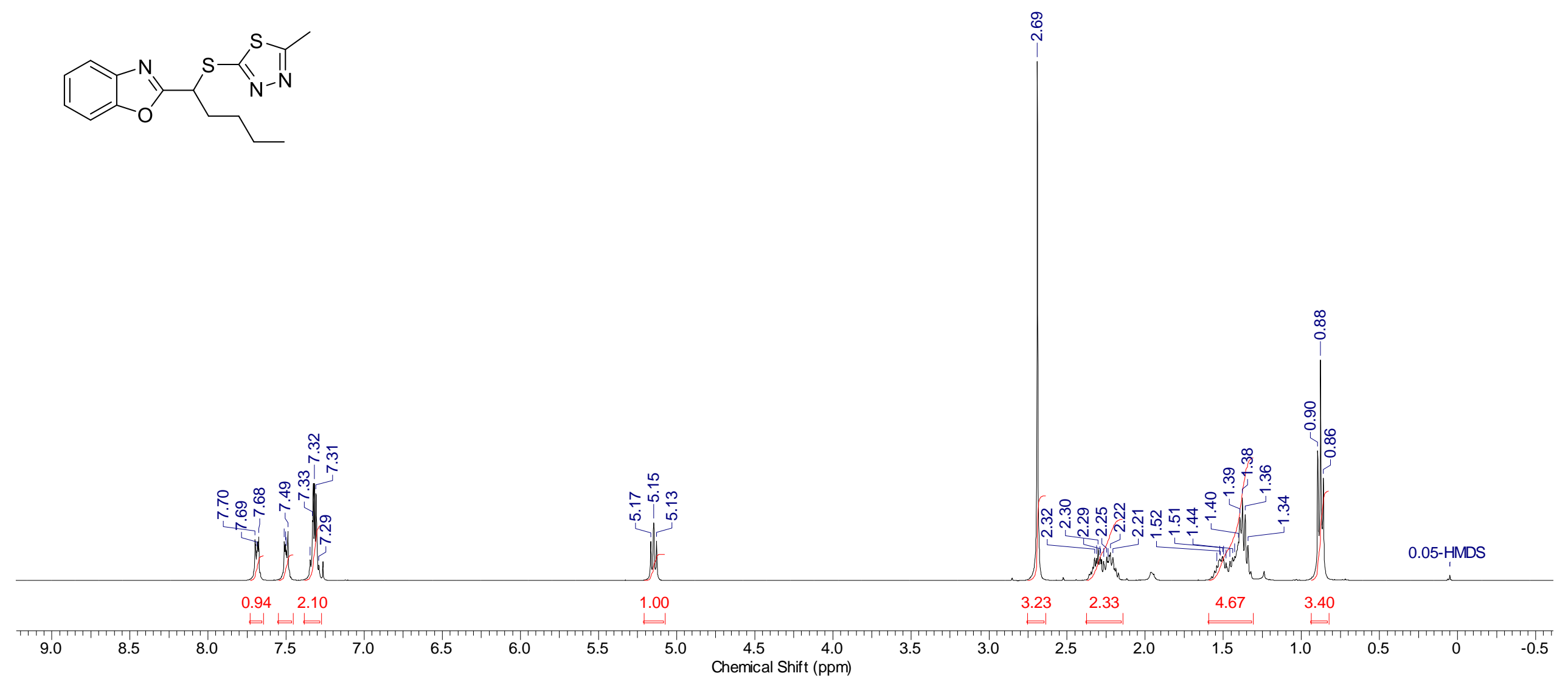


2-\{1-[(5-Methyl-1,3,4-thiadiazol-2-yl)thio]pentyl\}-1,3-benzoxazole (2e)

${ }^{13} \mathrm{C}$ NMR (100.6 MHz, $\mathrm{CDCl}_{3}$ )

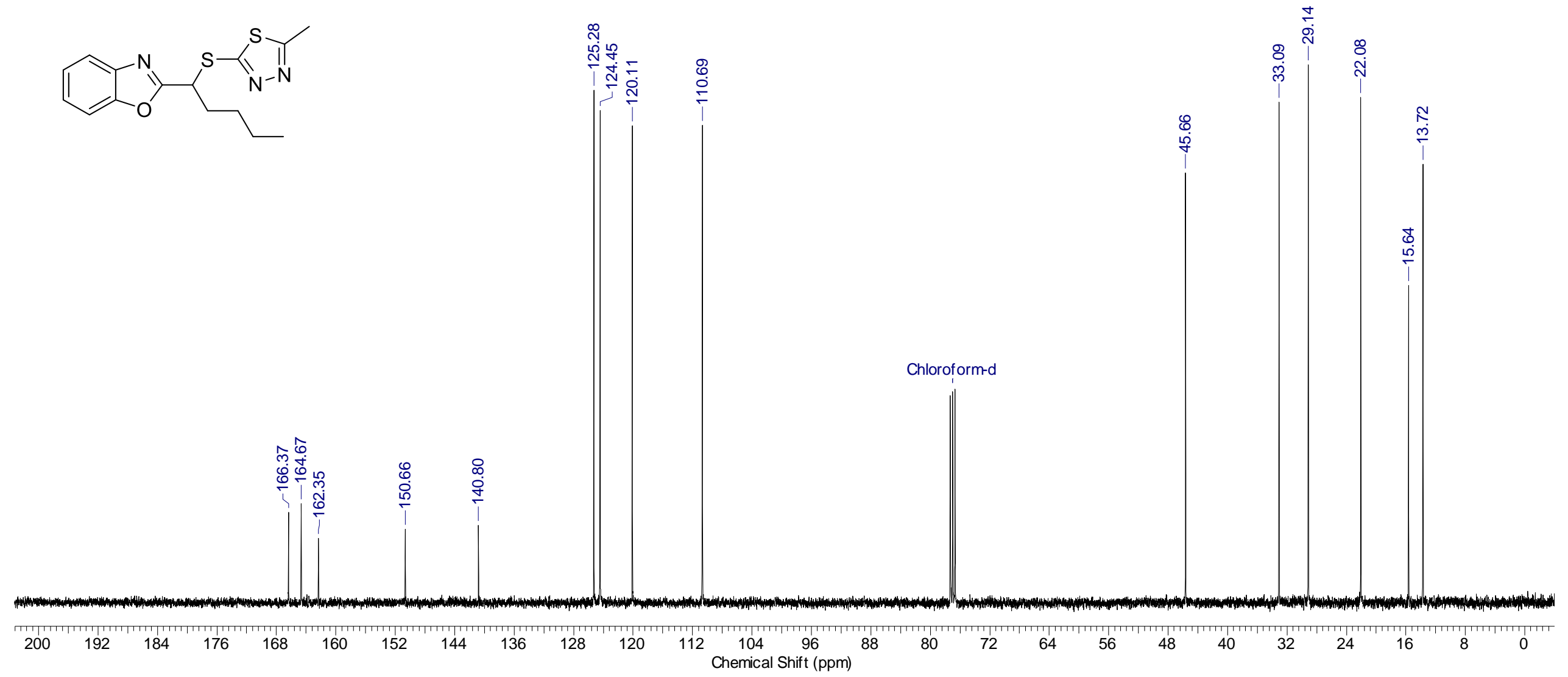




\section{5-\{[1-(1,3-Benzoxazol-2-yl)pentyl]thio\}-1,3,4-thiadiazol-2-amine (2f)}

${ }^{1} \mathrm{H}$ NMR (400 MHz, $\mathrm{CDCl}_{3}$ )

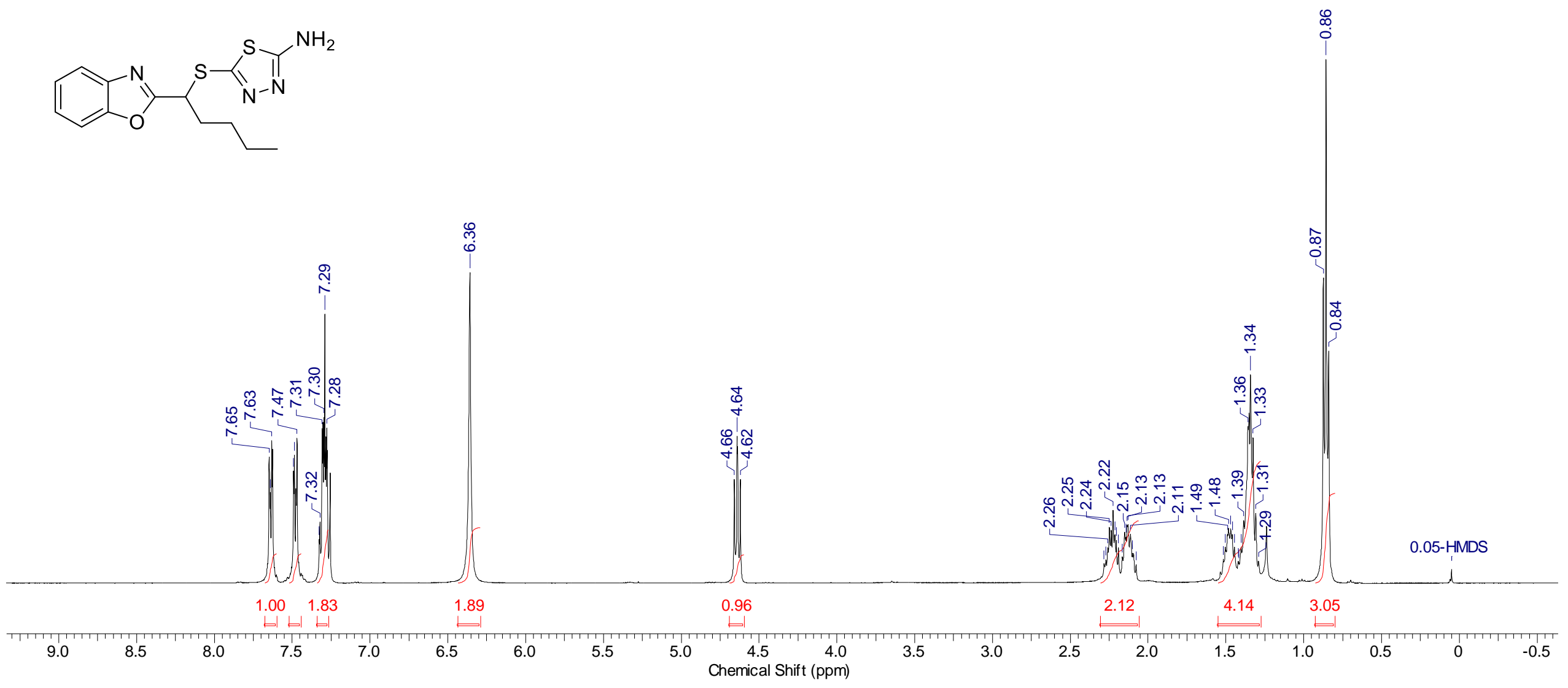




\section{5-\{[1-(1,3-Benzoxazol-2-yl)pentyl]thio\}-1,3,4-thiadiazol-2-amine (2f)}

${ }^{13} \mathrm{C}$ NMR (100.6 MHz, $\mathrm{CDCl}_{3}$ )

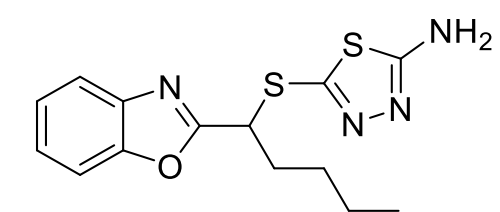

Chloroform-d

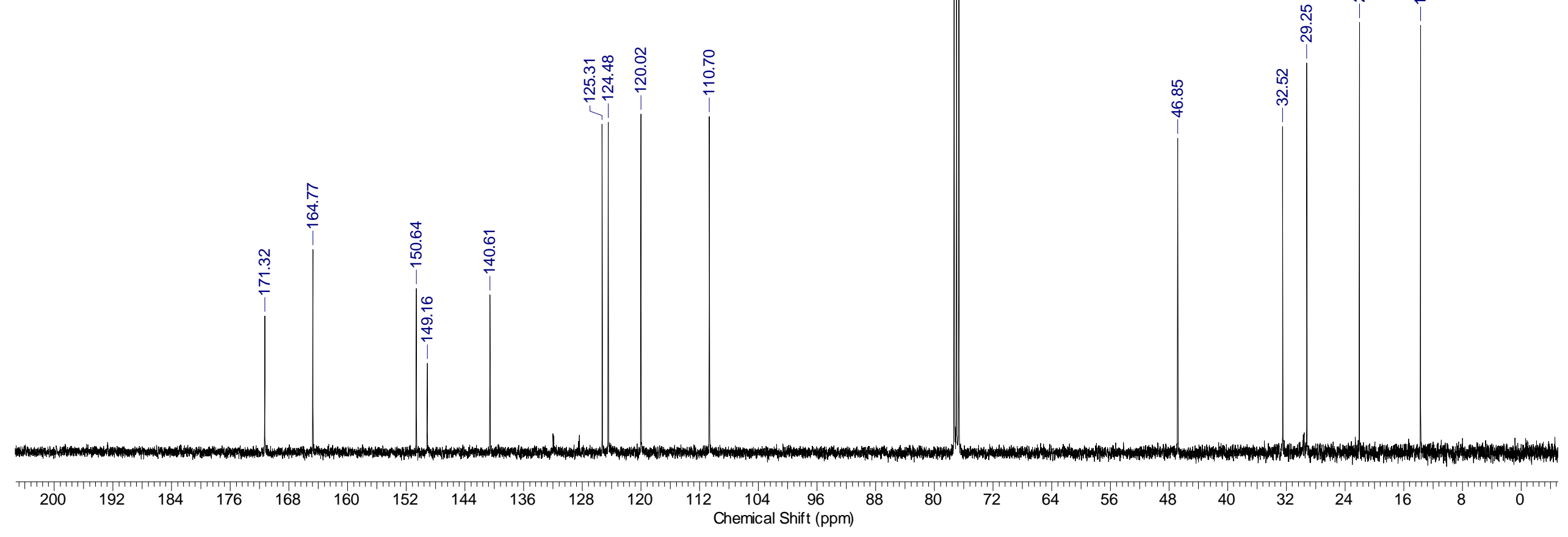


2-[1-(4,5-Dihydro-1,3-thiazol-2-ylthio)pentyl]-1,3-benzoxazole (2g)

${ }^{1} \mathrm{H}$ NMR $\left(600 \mathrm{MHz}, \mathrm{CDCl}_{3}\right)$

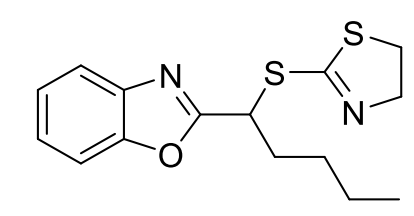


2-[1-(4,5-Dihydro-1,3-thiazol-2-ylthio)pentyl]-1,3-benzoxazole (2g)

${ }^{13} \mathrm{C}$ NMR (151 MHz, $\left.\mathrm{CDCl}_{3}\right)$

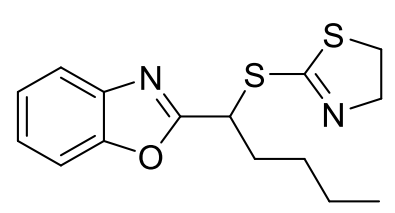

Chloroform-d

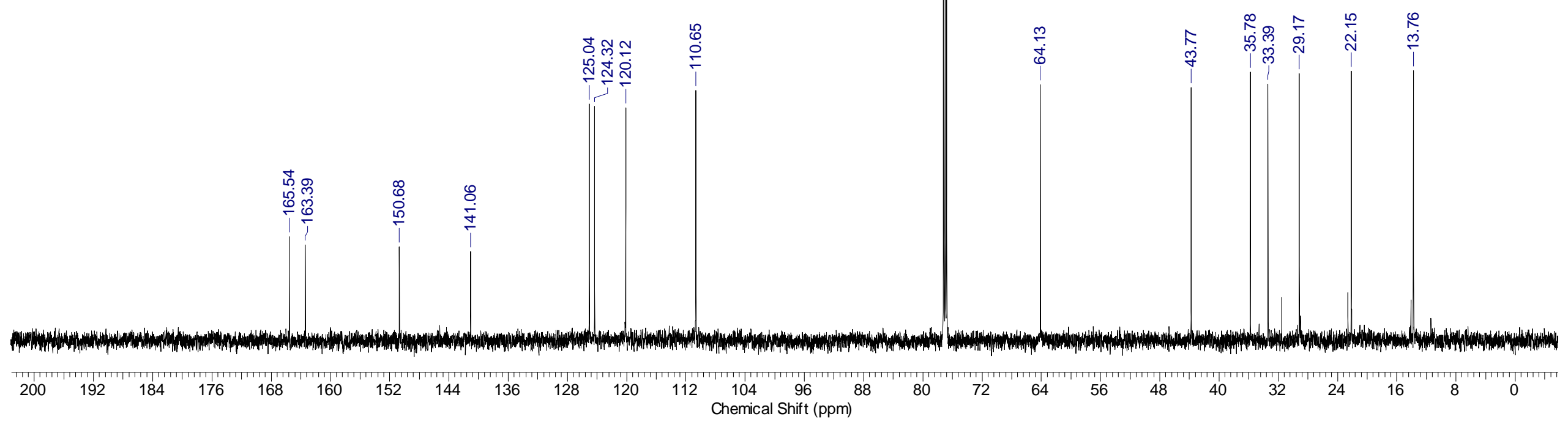


2-[1-(4,5-Dihydro-1,3-thiazol-2-ylthio)pentyl]-1,3-benzoxazole (2g)

${ }^{1} \mathrm{H}-{ }^{13} \mathrm{C}$ HMBC NMR

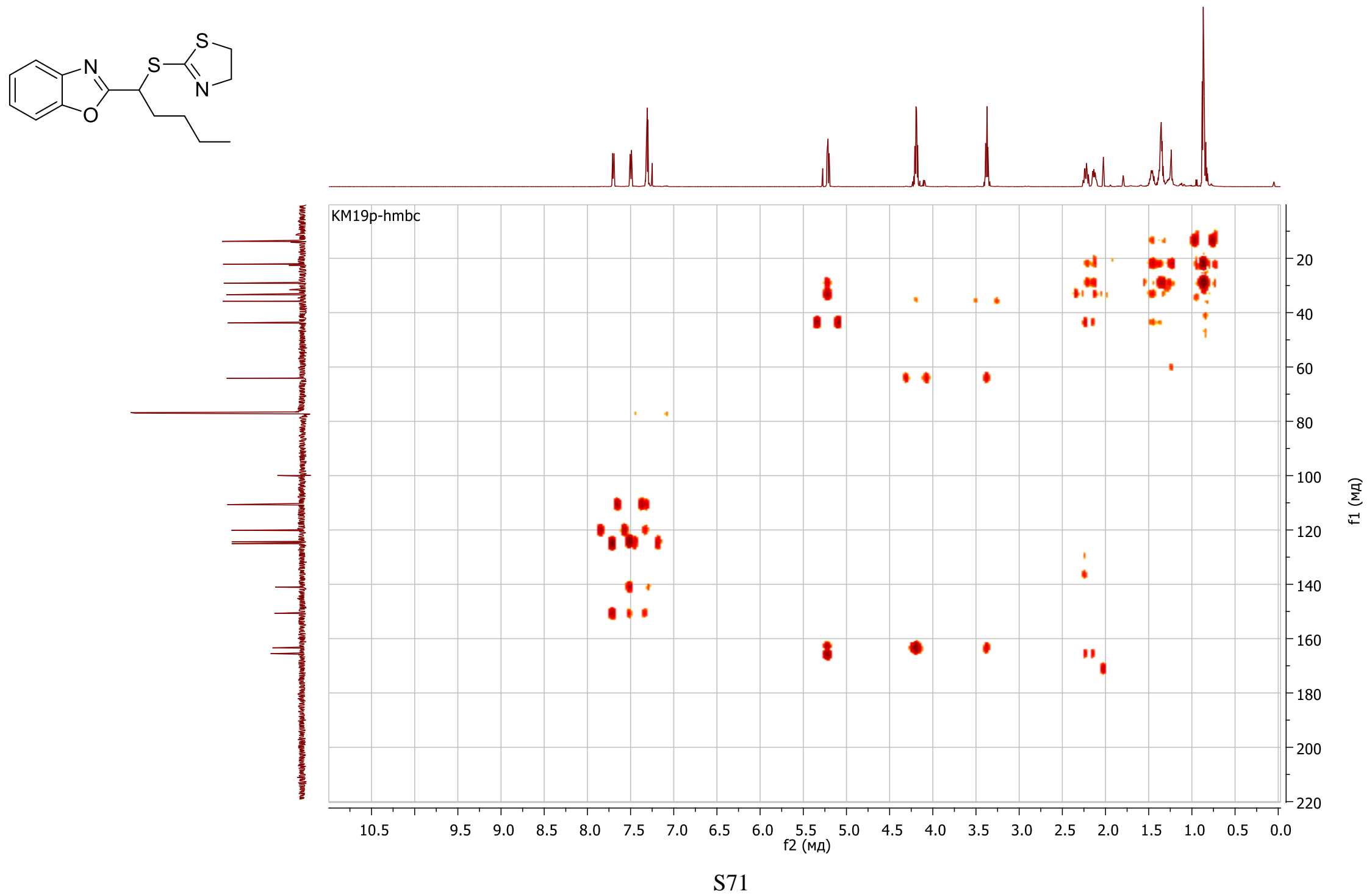




\section{2-\{1-[(1-Methyl-1H-imidazol-2-yl)thio]pentyl\}-1,3-benzoxazole (2h)}

${ }^{1} \mathrm{H}$ NMR $\left(400 \mathrm{MHz}, \mathrm{CDCl}_{3}\right)$
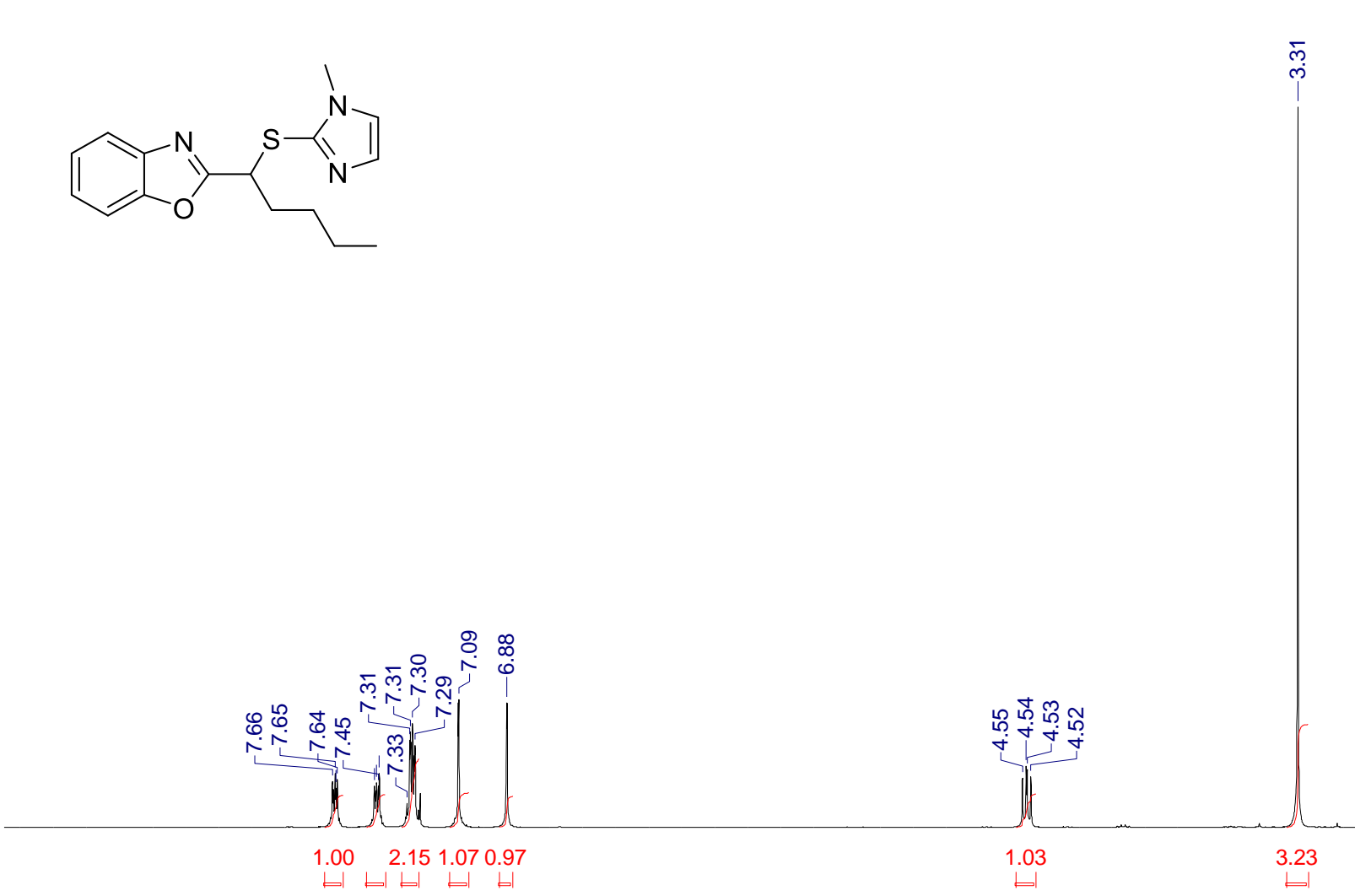

$\begin{array}{lllllllllll}7.0 & 8.5 & 8.0 & 7.5 & 7.0 & 6.5 & 6.0 & 5.5 & 5.0 & 4.5 & 4.0 \\ \text { Chemical Shift (ppm) }\end{array}$




\section{2-\{1-[(1-Methyl-1H-imidazol-2-yl)thio]pentyl\}-1,3-benzoxazole (2h)}

${ }^{13} \mathrm{C}$ NMR (100.6 MHz, $\mathrm{CDCl}_{3}$ )

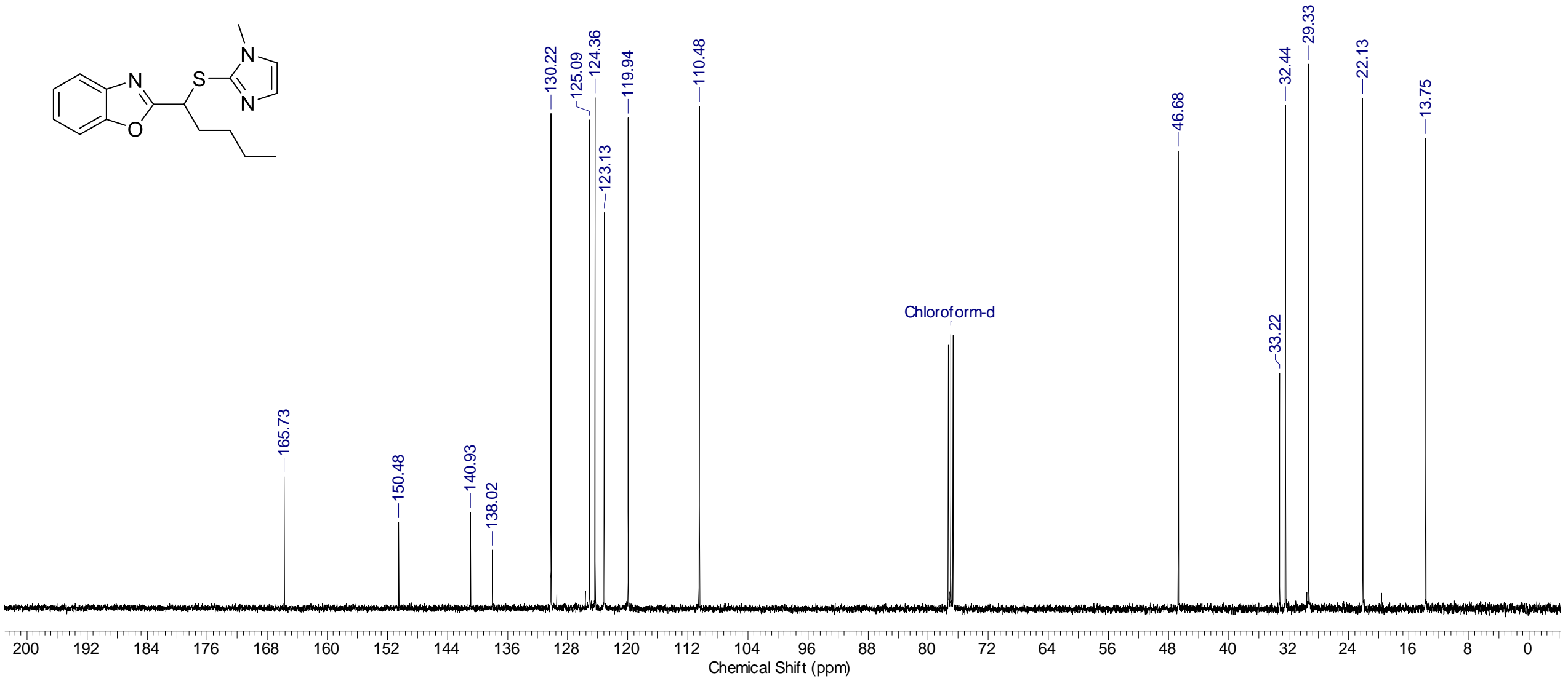




\section{2-\{1-[(1-Methyl-1H-tetrazol-5-yl)thio]pentyl\}-1,3-benzoxazole (2i)}

${ }^{1} \mathrm{H}$ NMR (400 MHz, $\mathrm{CDCl}_{3}$ )

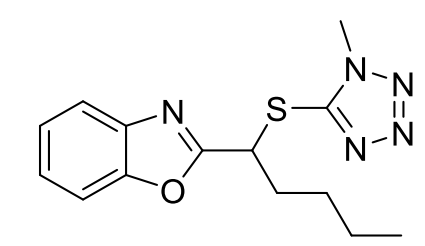

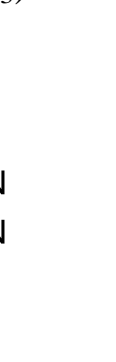


2-\{1-[(1-Methyl-1H-tetrazol-5-yl)thio]pentyl\}-1,3-benzoxazole (2i)

${ }^{13} \mathrm{C}$ NMR (100.6 MHz, $\left.\mathrm{CDCl}_{3}\right)$

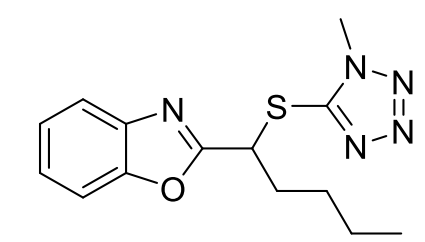

Chloroform-d

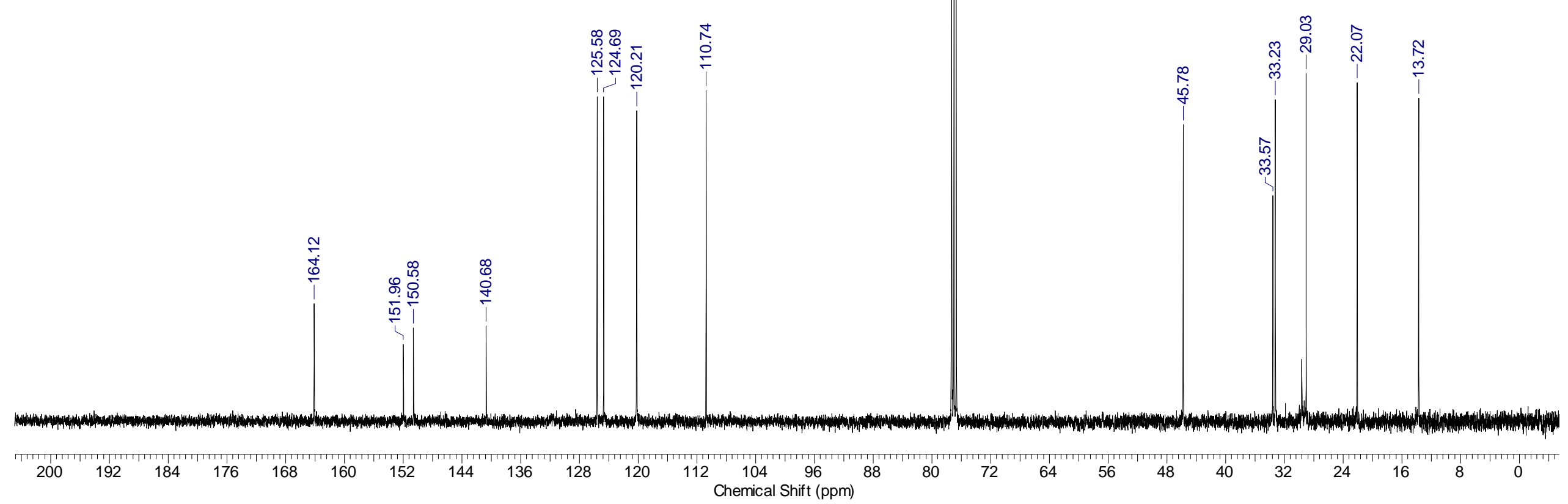


2-(1-\{[4-(4-Methoxyphenyl)-5-(4-nitrophenyl)-4H-1,2,4-triazol-3-yl]thio\}pentyl)-1,3-benzoxazole (2j)

${ }^{1} \mathrm{H}$ NMR $\left(600 \mathrm{MHz}, \mathrm{CDCl}_{3}\right)$

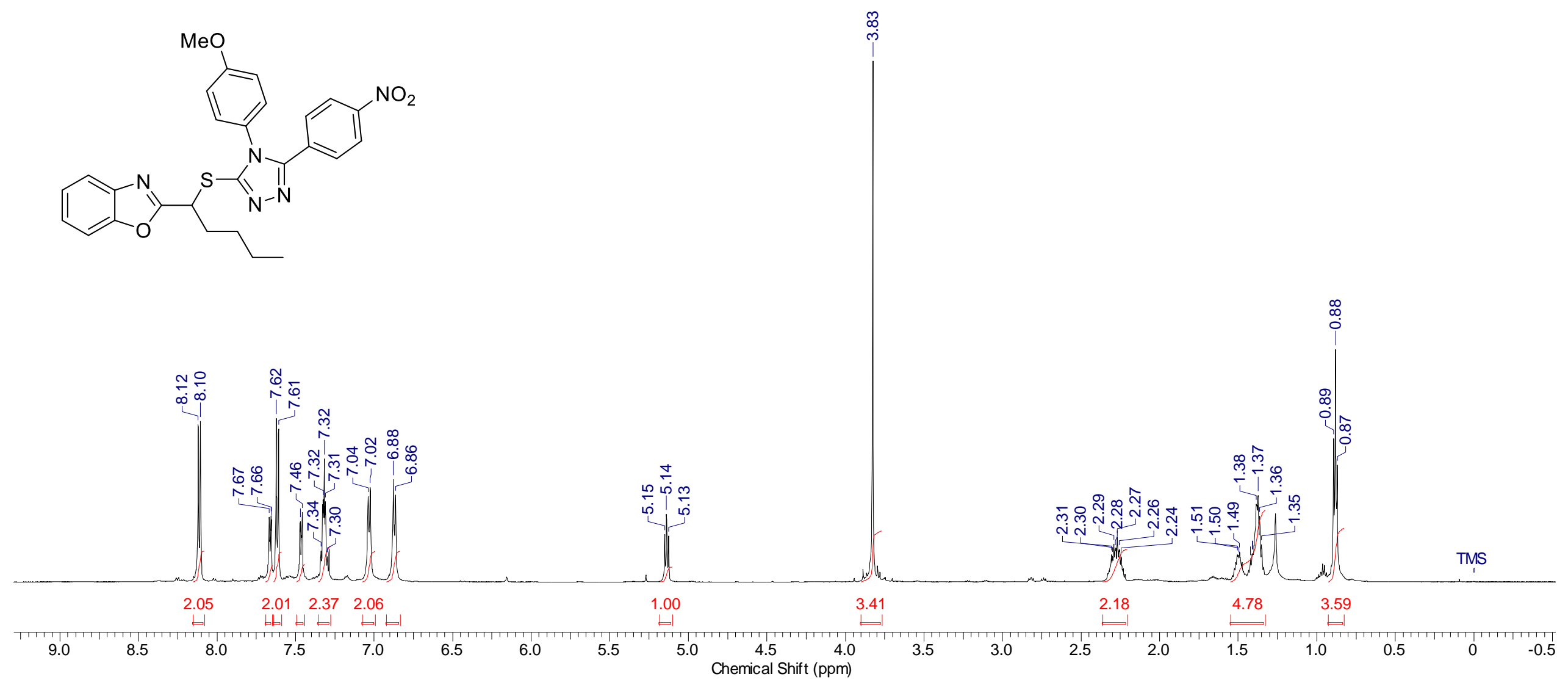


2-(1-\{[4-(4-Methoxyphenyl)-5-(4-nitrophenyl)-4H-1,2,4-triazol-3-yl]thio\}pentyl)-1,3-benzoxazole (2j)

${ }^{13} \mathrm{C}$ NMR (151 MHz, $\left.\mathrm{CDCl}_{3}\right)$

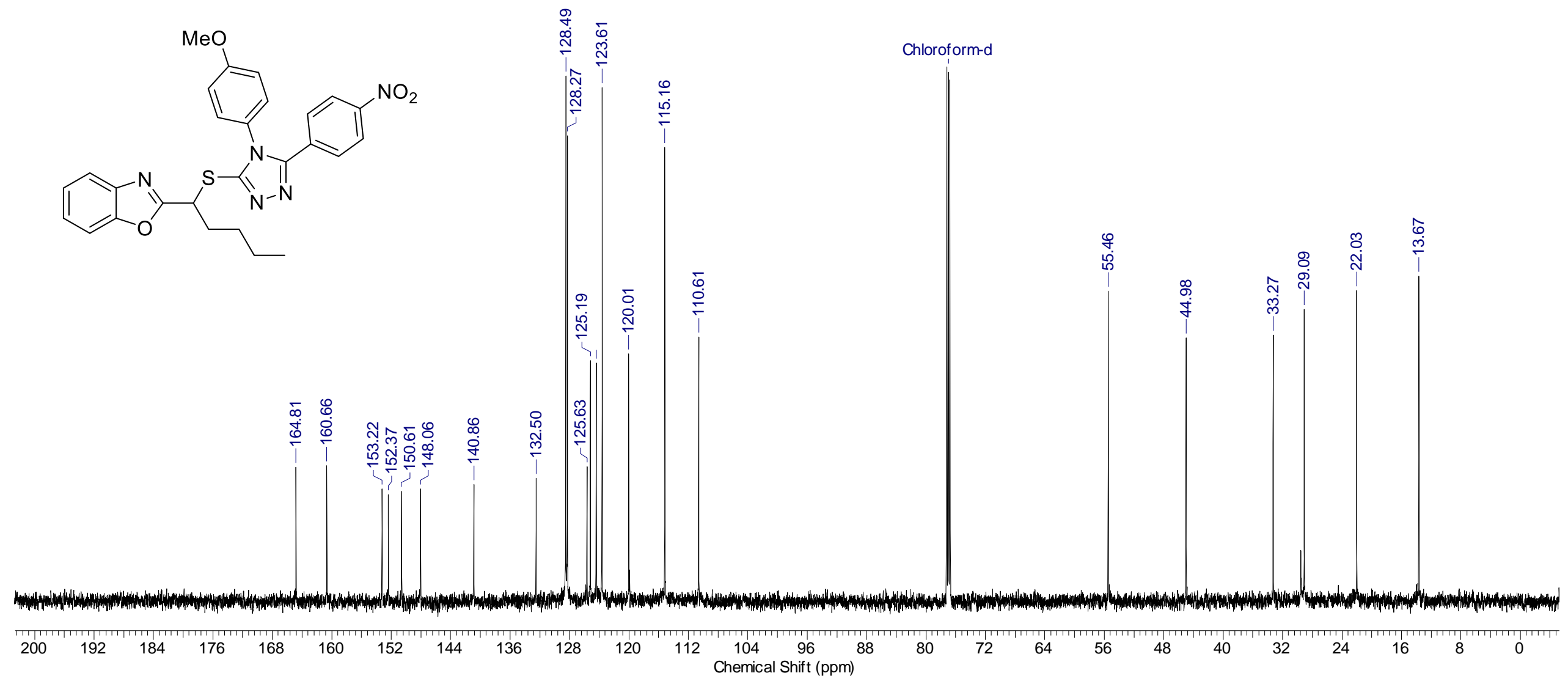


2-(1-\{[5-(4-Nitrophenyl)-4-(tetrahydrofuran-2-ylmethyl)-4H-1,2,4-triazol-3-yl]thio\}pentyl)-1,3-benzoxazole (2k)

${ }^{1} \mathrm{H}$ NMR (400 MHz, $\mathrm{CDCl}_{3}$ )

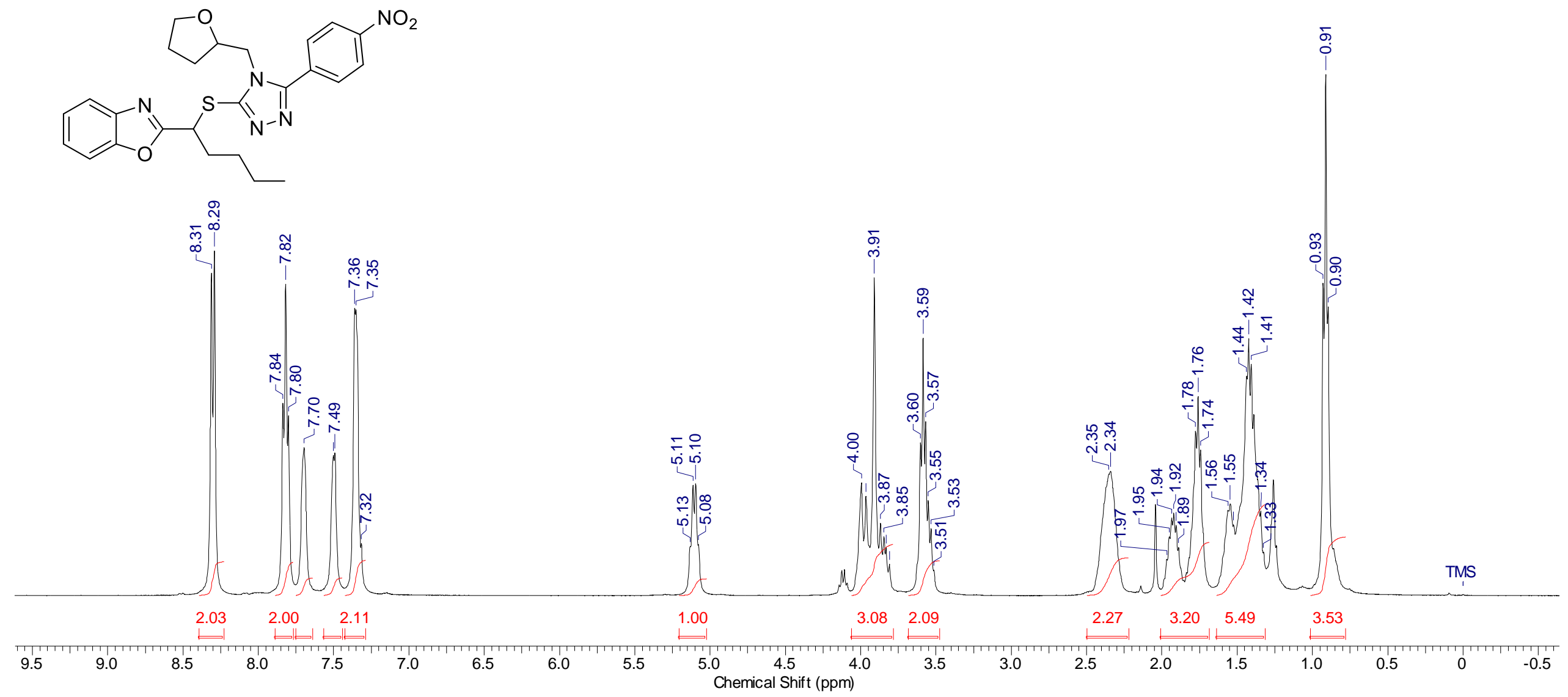


2-(1-\{[5-(4-Nitrophenyl)-4-(tetrahydrofuran-2-ylmethyl)-4H-1,2,4-triazol-3-yl]thio\}pentyl)-1,3-benzoxazole (2k)

${ }^{13} \mathrm{C}$ NMR (100.6 MHz, $\mathrm{CDCl}_{3}$ )

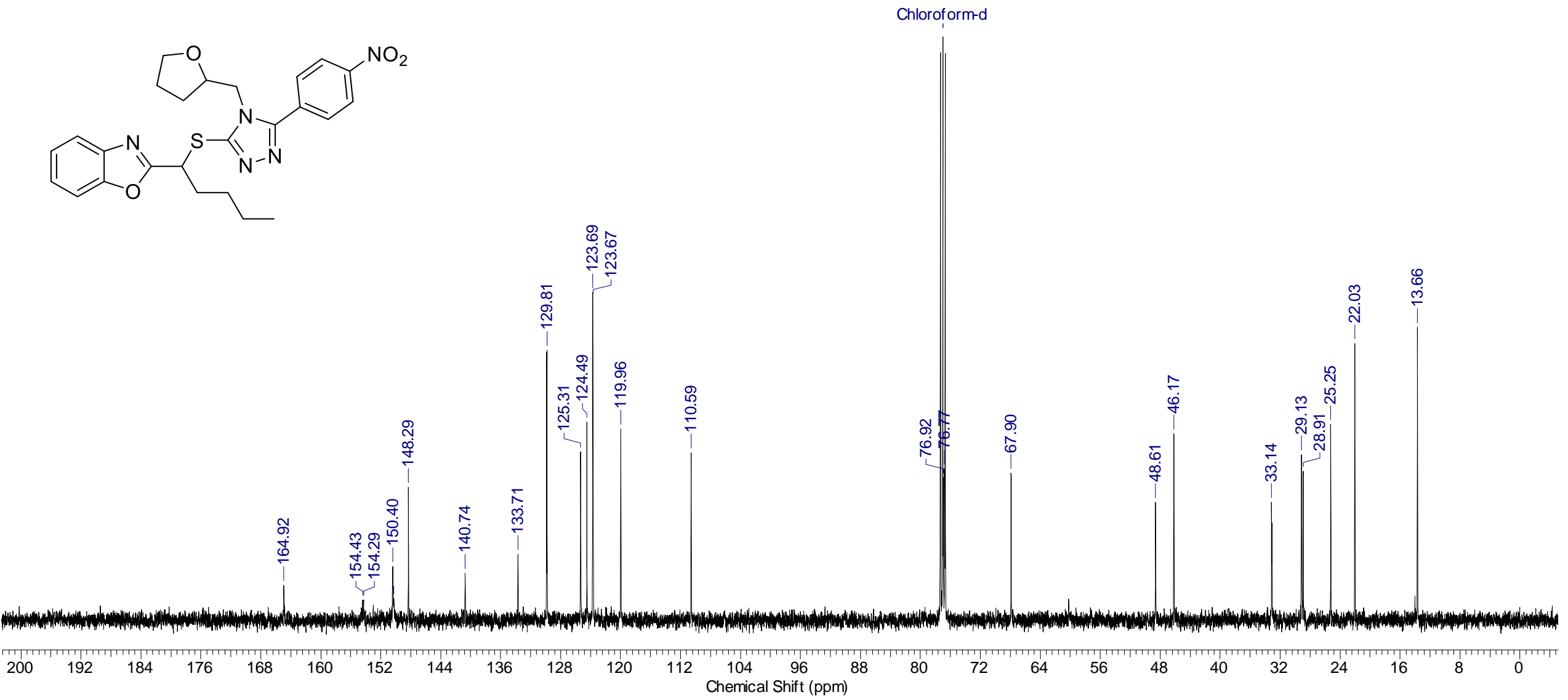




\section{2-[1-(Pyridin-2-ylthio)pentyl]-1,3-benzoxazole (2I)}

${ }^{1} \mathrm{H}$ NMR (400 MHz, $\mathrm{CDCl}_{3}$ )

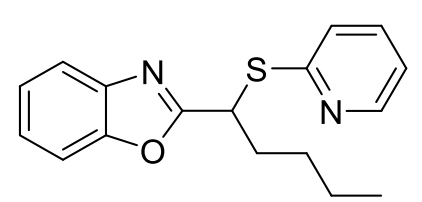

$$
\text { (1) }
$$




\section{2-[1-(Pyridin-2-ylthio)pentyl]-1,3-benzoxazole (2l)}

${ }^{13} \mathrm{C}$ NMR (100.6 MHz, $\mathrm{CDCl}_{3}$ )

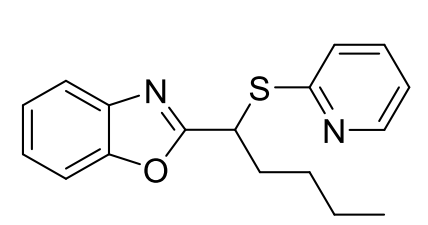

$\stackrel{\stackrel{8}{\circ}}{\stackrel{\circ}{+}}$

Chloroform-d

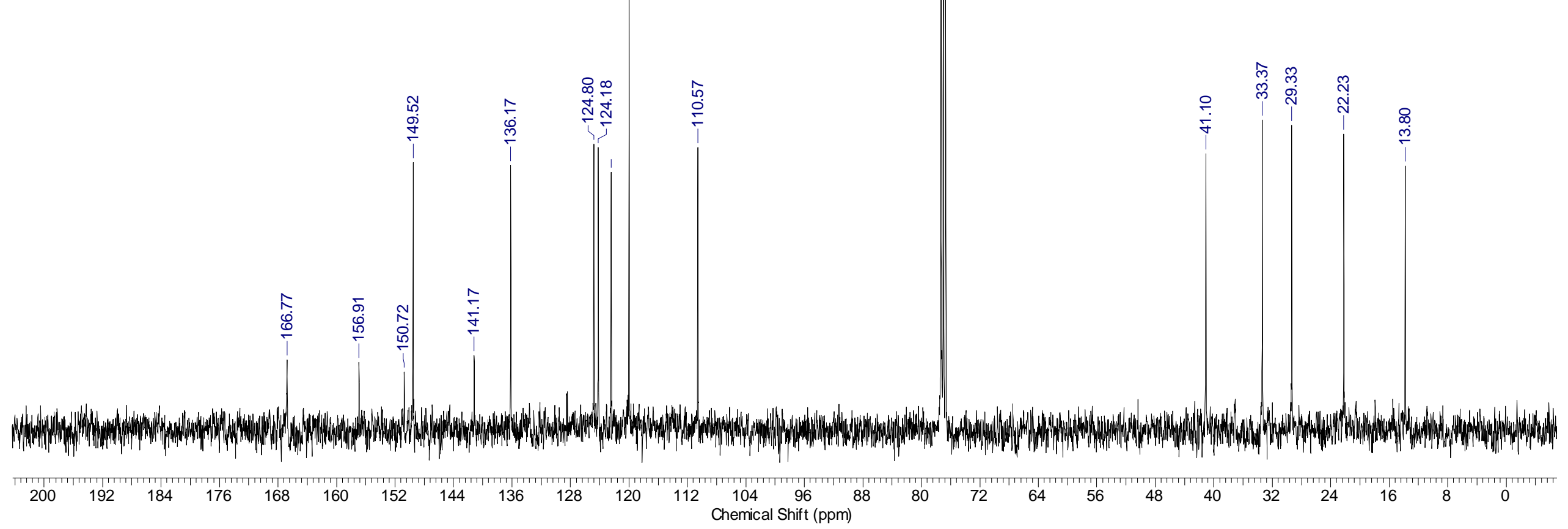




\section{3-\{[1-(1,3-Benzoxazol-2-yl)pentyl] thio $\}-5 H-[1,2,4]$ triazino[5,6-b]indole (2n)}

${ }^{1} \mathrm{H}$ NMR (400 MHz, $\left.\mathrm{CDCl}_{3}-\mathrm{CD}_{3} \mathrm{OD}\right)$

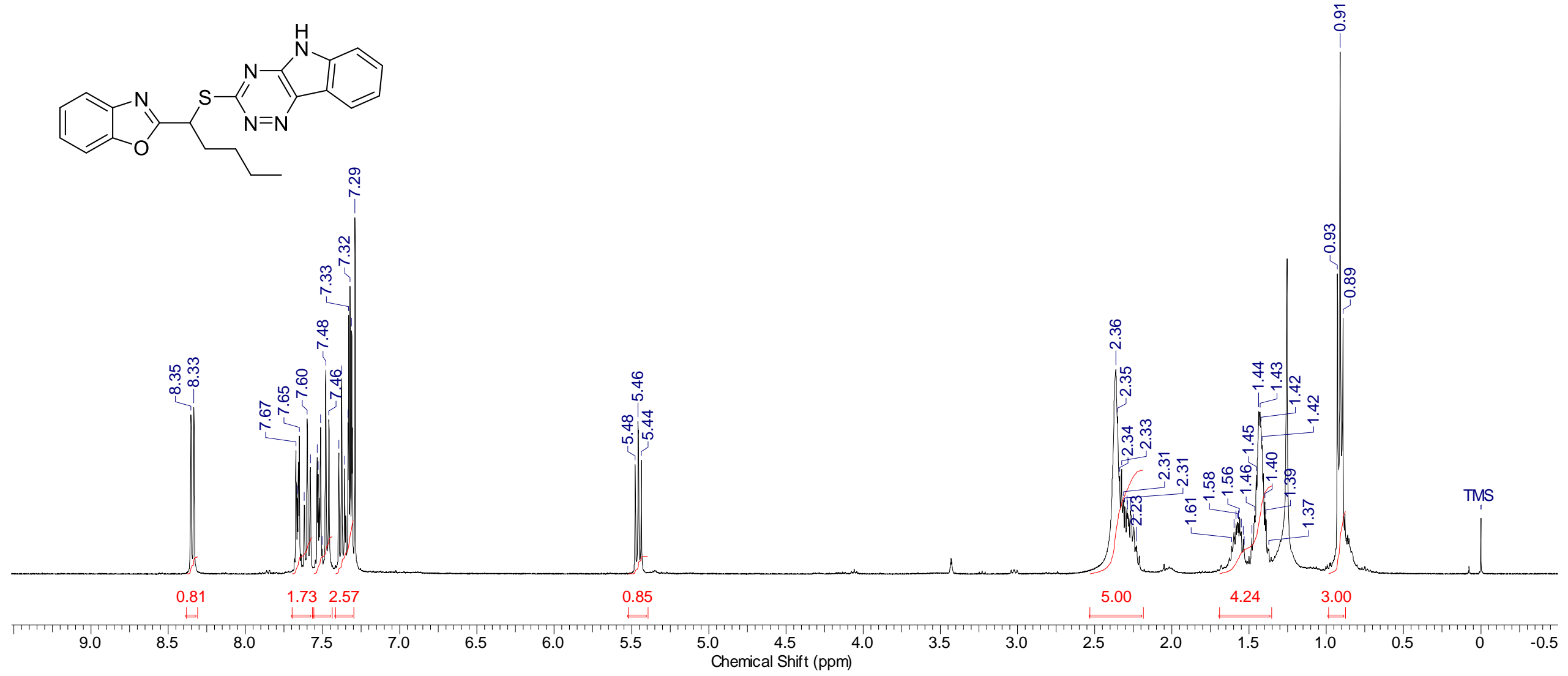




\section{3-\{[1-(1,3-Benzoxazol-2-yl)pentyl]thio $\}-5 H-[1,2,4]$ triazino[5,6-b]indole (2n)}

${ }^{13} \mathrm{C}$ NMR (100.6 MHz, $\left.\mathrm{CDCl}_{3}-\mathrm{CD}_{3} \mathrm{OD}\right)$
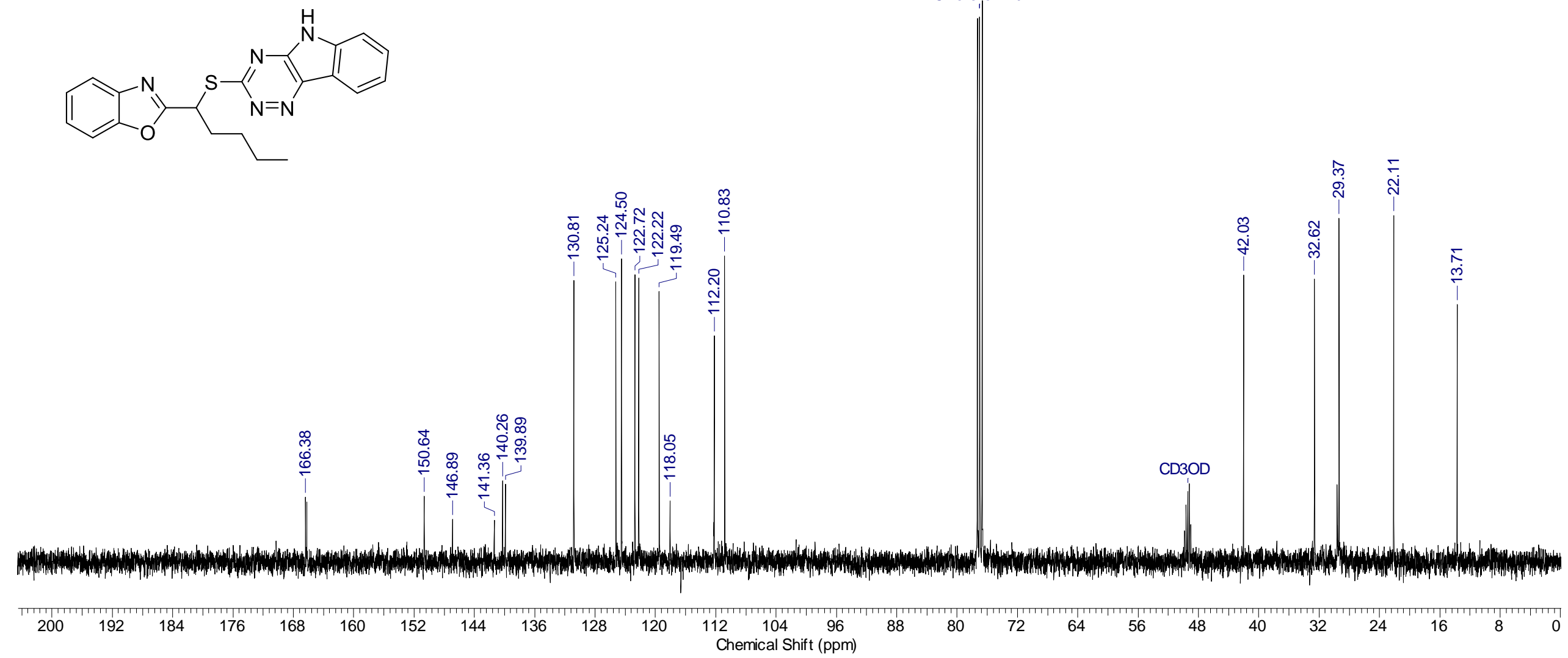
2-\{1-[(4-Bromophenyl)thio]pentyl\}-1,3-benzoxazole (2o)

${ }^{1} \mathrm{H}$ NMR (400 MHz, $\mathrm{CDCl}_{3}$ )

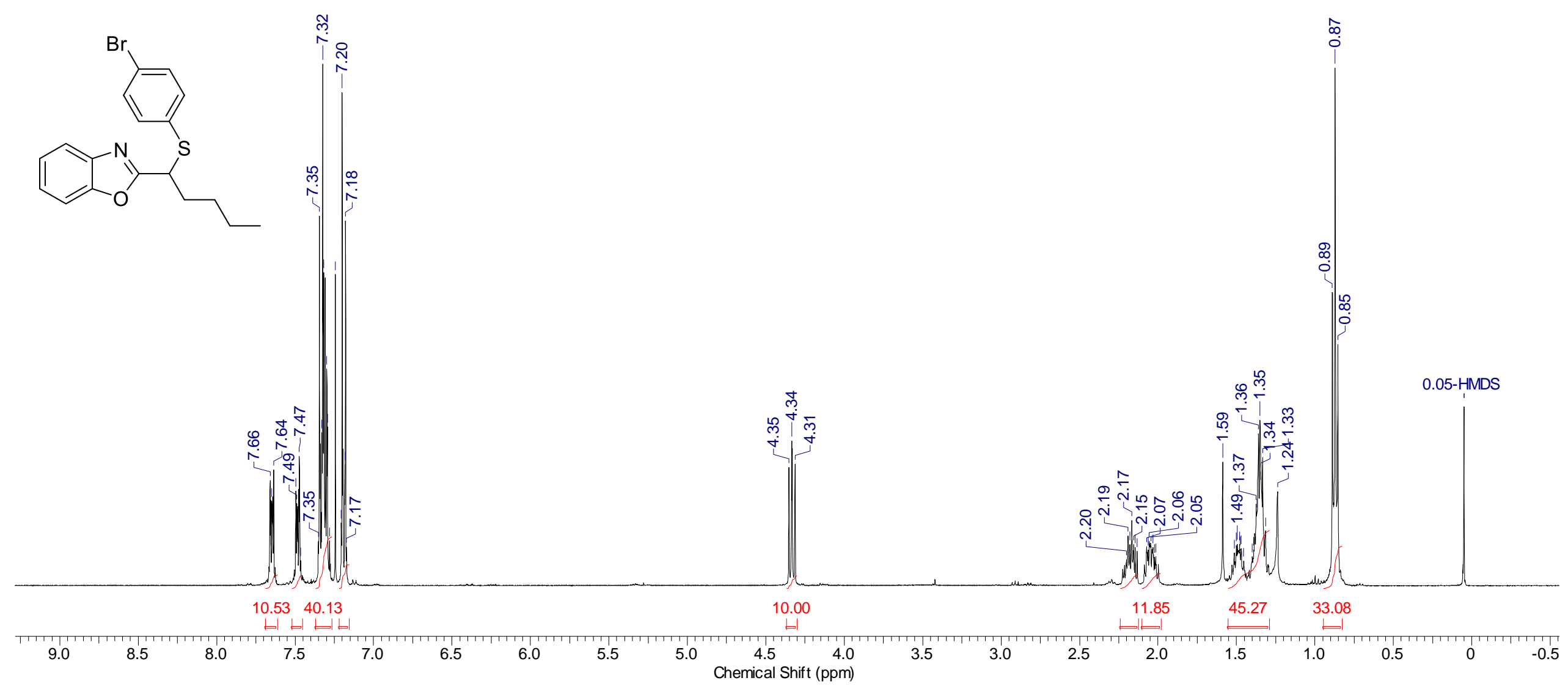




\section{2-\{1-[(4-Bromophenyl)thio]pentyl\}-1,3-benzoxazole (2o)}

${ }^{13} \mathrm{C}$ NMR (100.6 MHz, $\mathrm{CDCl}_{3}$ )

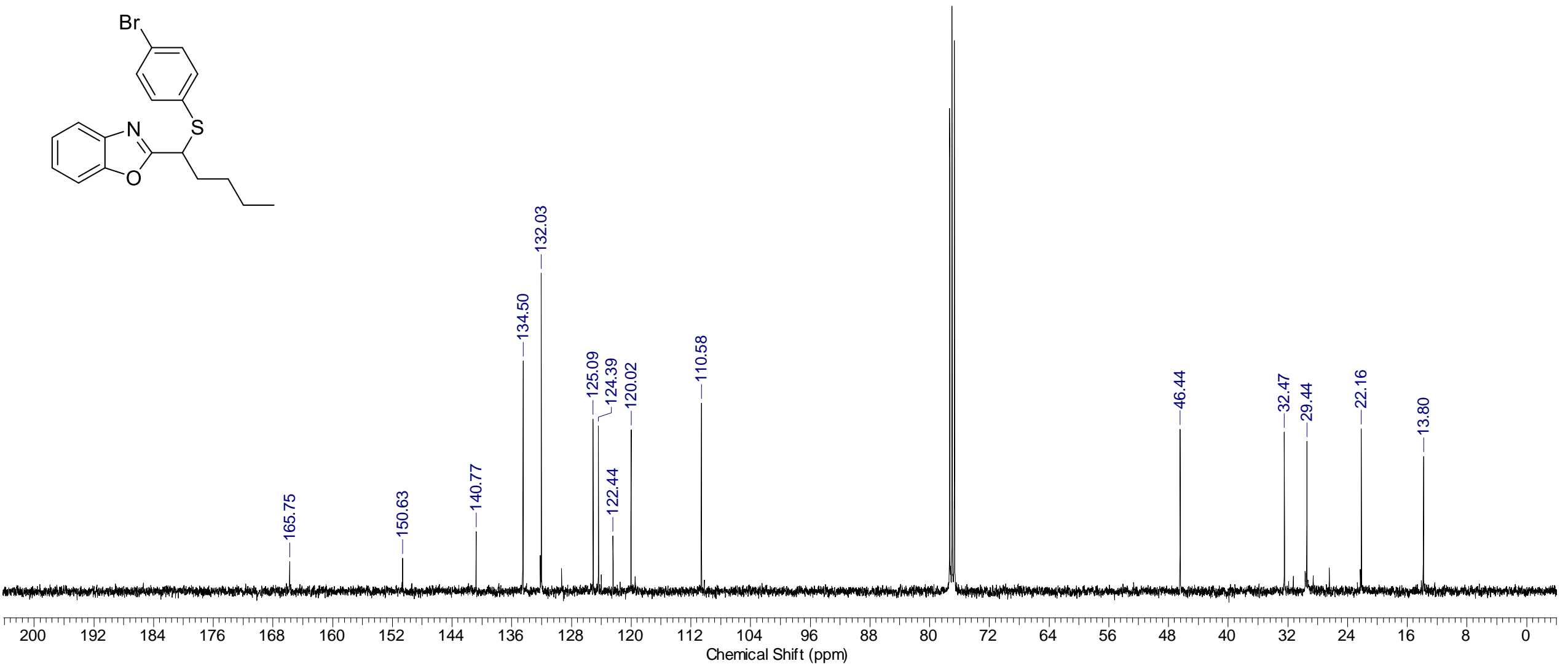




\section{2-[1-(Phenylthio)pentyl]-1,3-benzoxazole (2p)}

${ }^{1} \mathrm{H}$ NMR (600 MHz, $\mathrm{CDCl}_{3}$ )

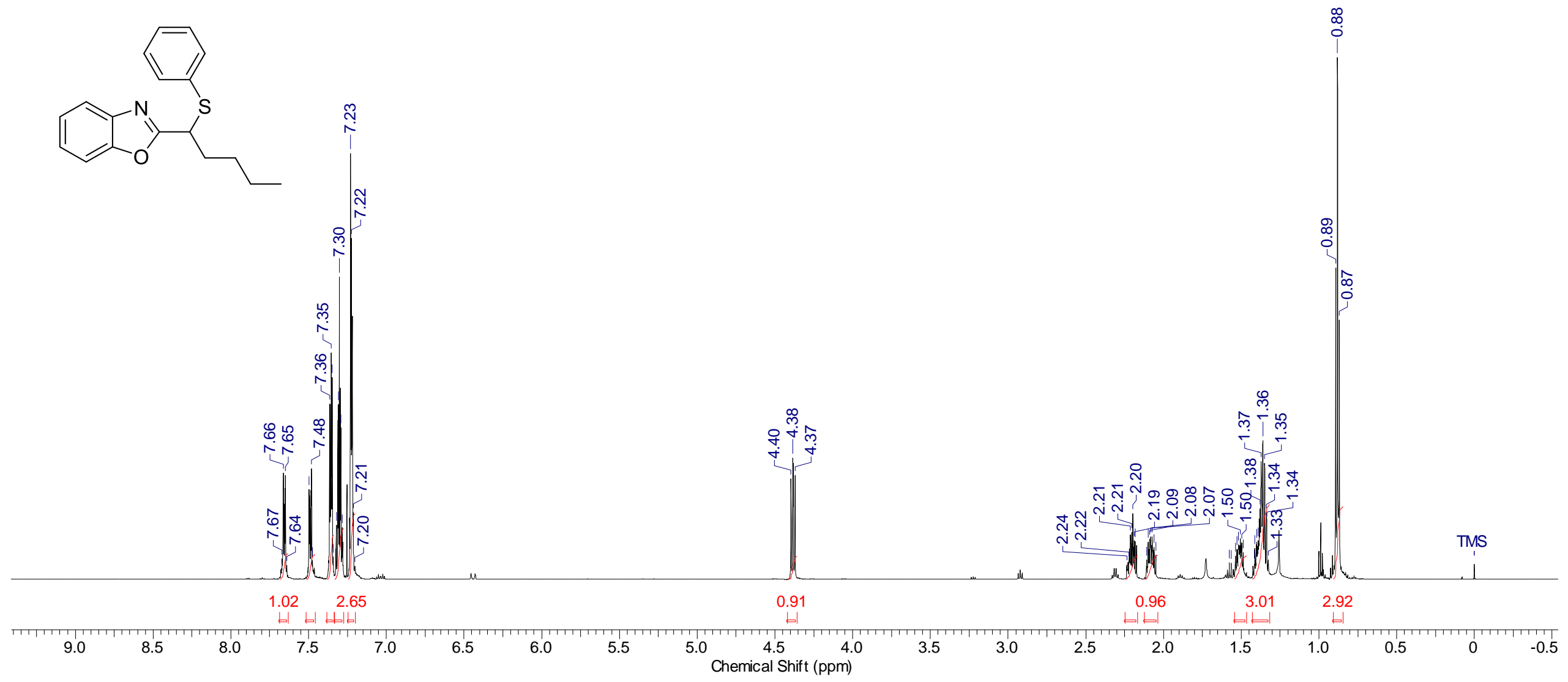


2-[1-(Phenylthio)pentyl]-1,3-benzoxazole (2p)

${ }^{13} \mathrm{C}$ NMR (151 MHz, $\left.\mathrm{CDCl}_{3}\right)$
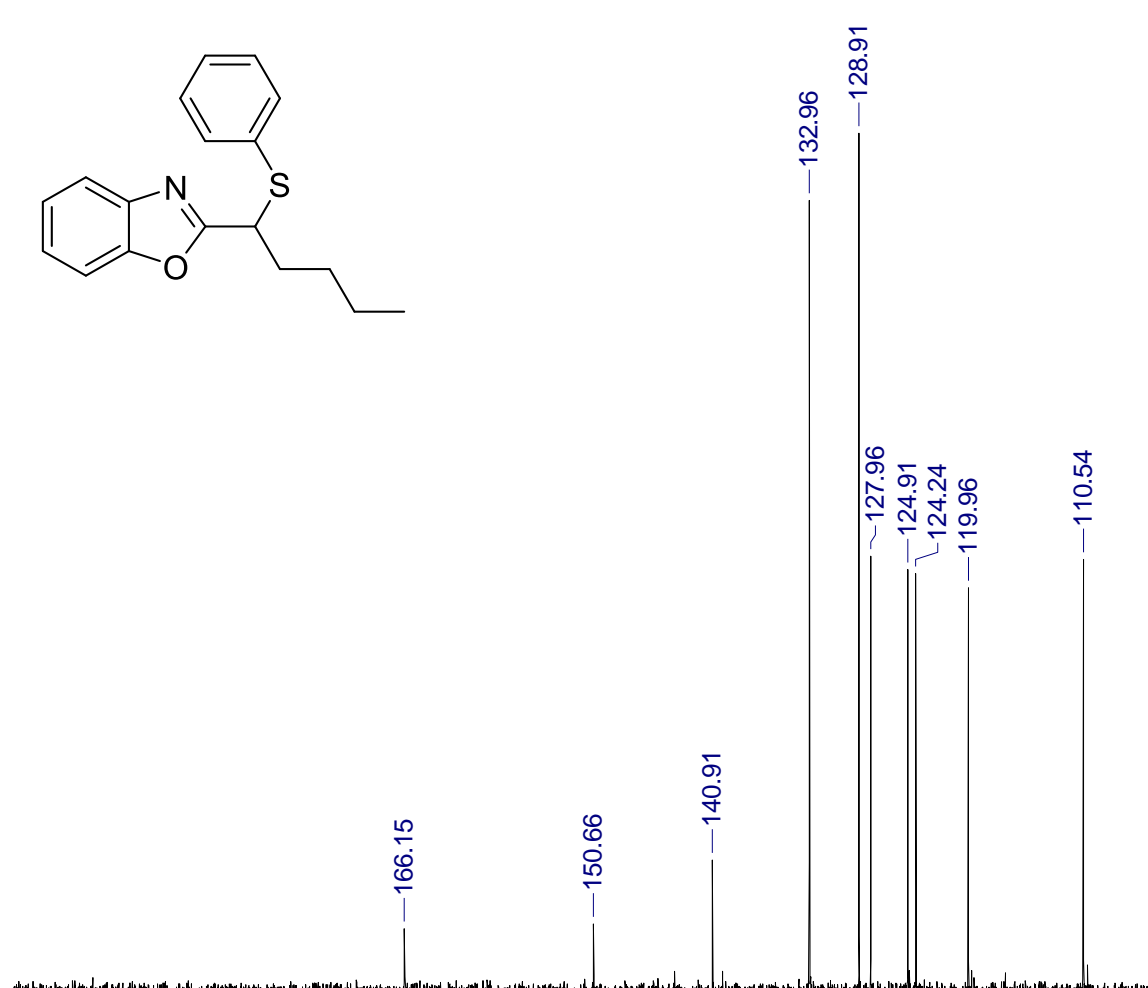

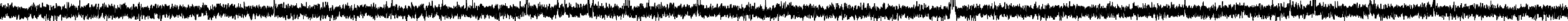

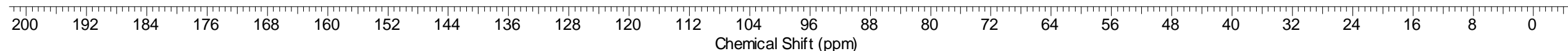


5-Methyl-2-\{1-[(5-methyl-1,3,4-oxadiazol-2-yl)thio]pentyl\}-1,3-benzoxazole (2q)

${ }^{1} \mathrm{H}$ NMR $\left(400 \mathrm{MHz}, \mathrm{CDCl}_{3}\right)$

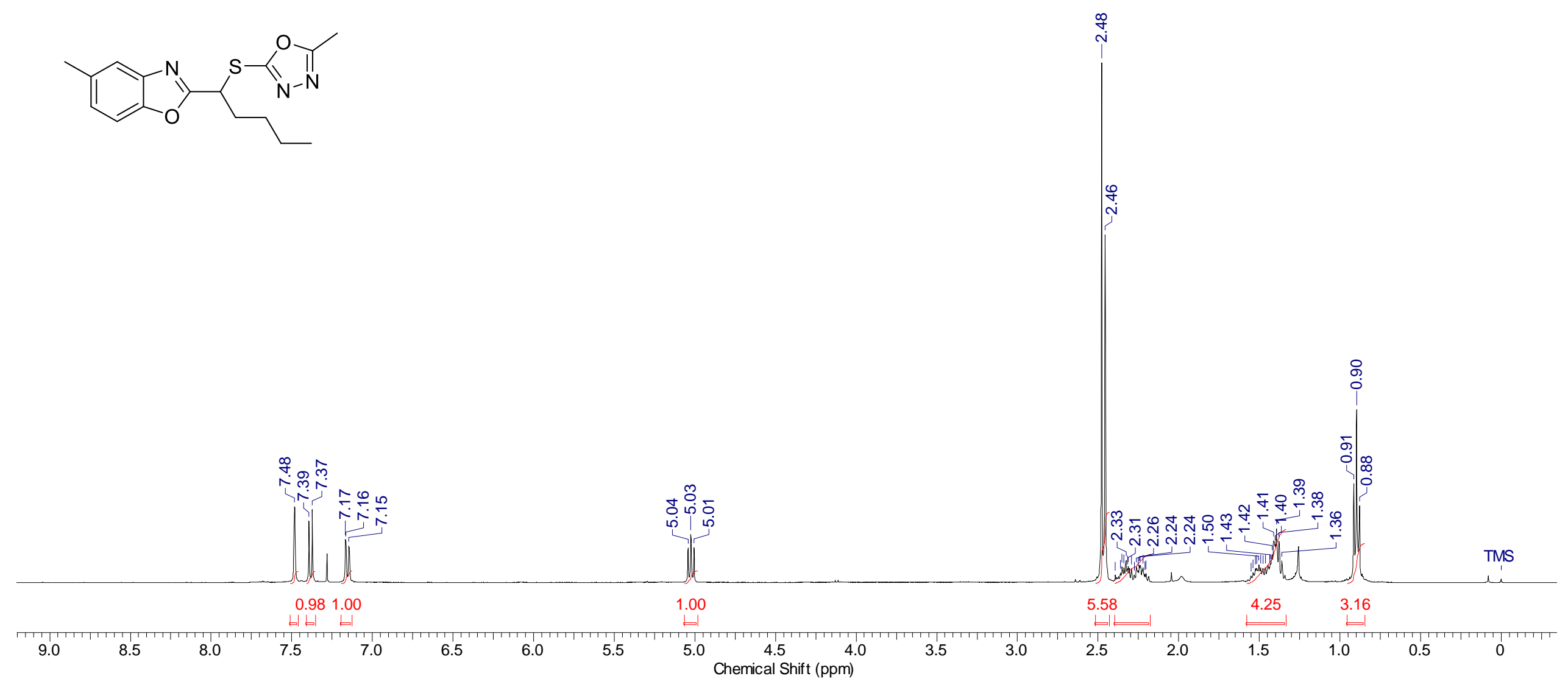


5-Methyl-2-\{1-[(5-methyl-1,3,4-oxadiazol-2-yl)thio]pentyl\}-1,3-benzoxazole (2q)

${ }^{13} \mathrm{C}$ NMR (100.6 MHz, $\left.\mathrm{CDCl}_{3}\right)$

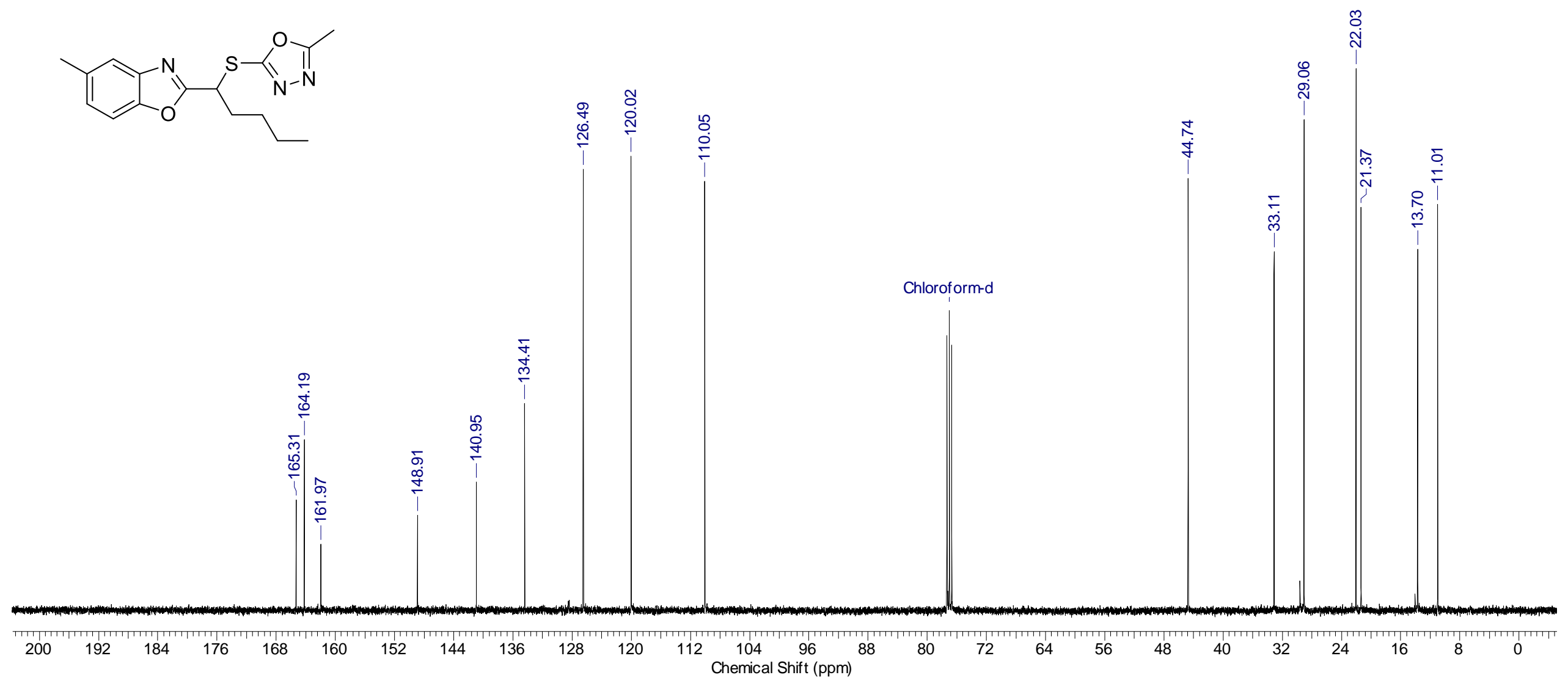


Methyl 2-\{1-[(5-methyl-1,3,4-oxadiazol-2-yl)thio]pentyl\}-1,3-benzoxazole-5-carboxylate (2r)

${ }^{1} \mathrm{H}$ NMR (400 MHz, $\left.\mathrm{CDCl}_{3}\right)$

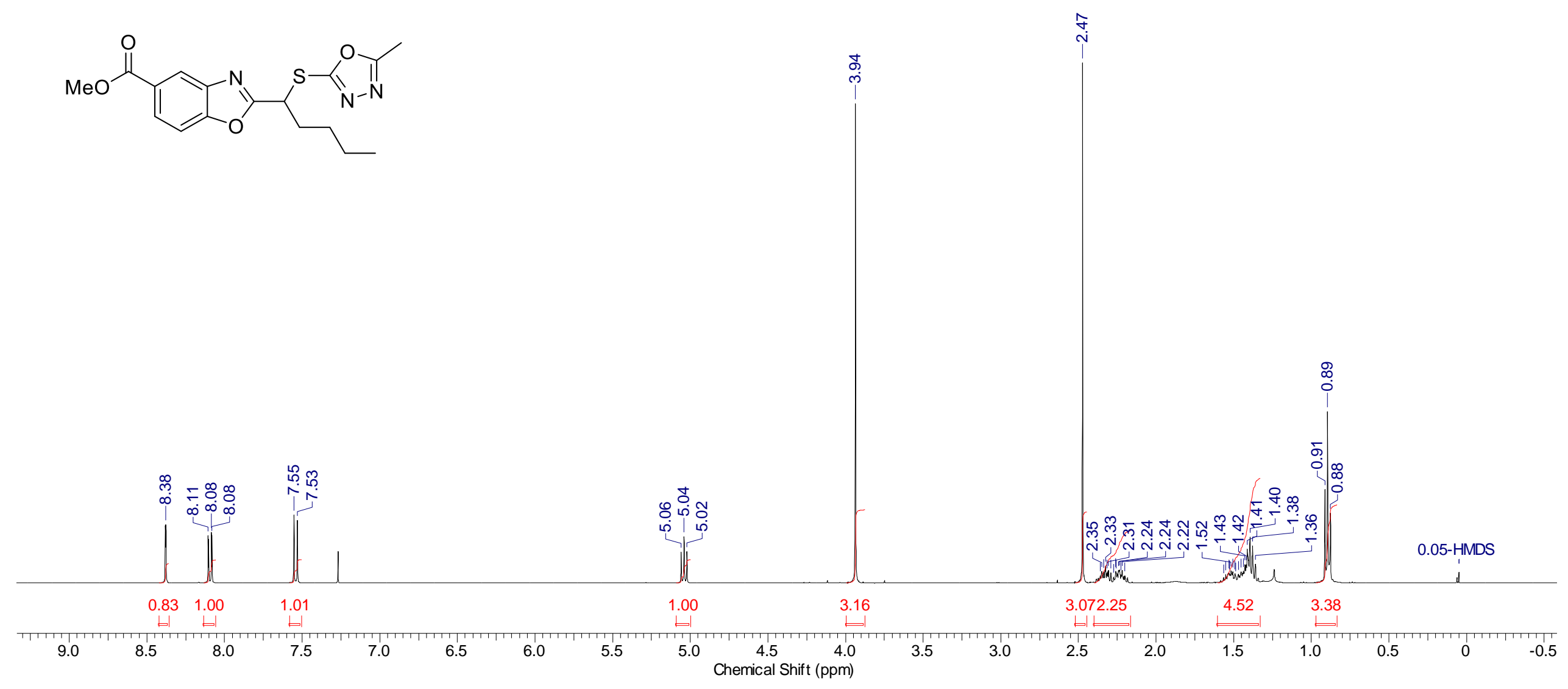


Methyl 2-\{1-[(5-methyl-1,3,4-oxadiazol-2-yl)thio]pentyl\}-1,3-benzoxazole-5-carboxylate (2r)

${ }^{13} \mathrm{C}$ NMR (100.6 MHz, $\left.\mathrm{CDCl}_{3}\right)$

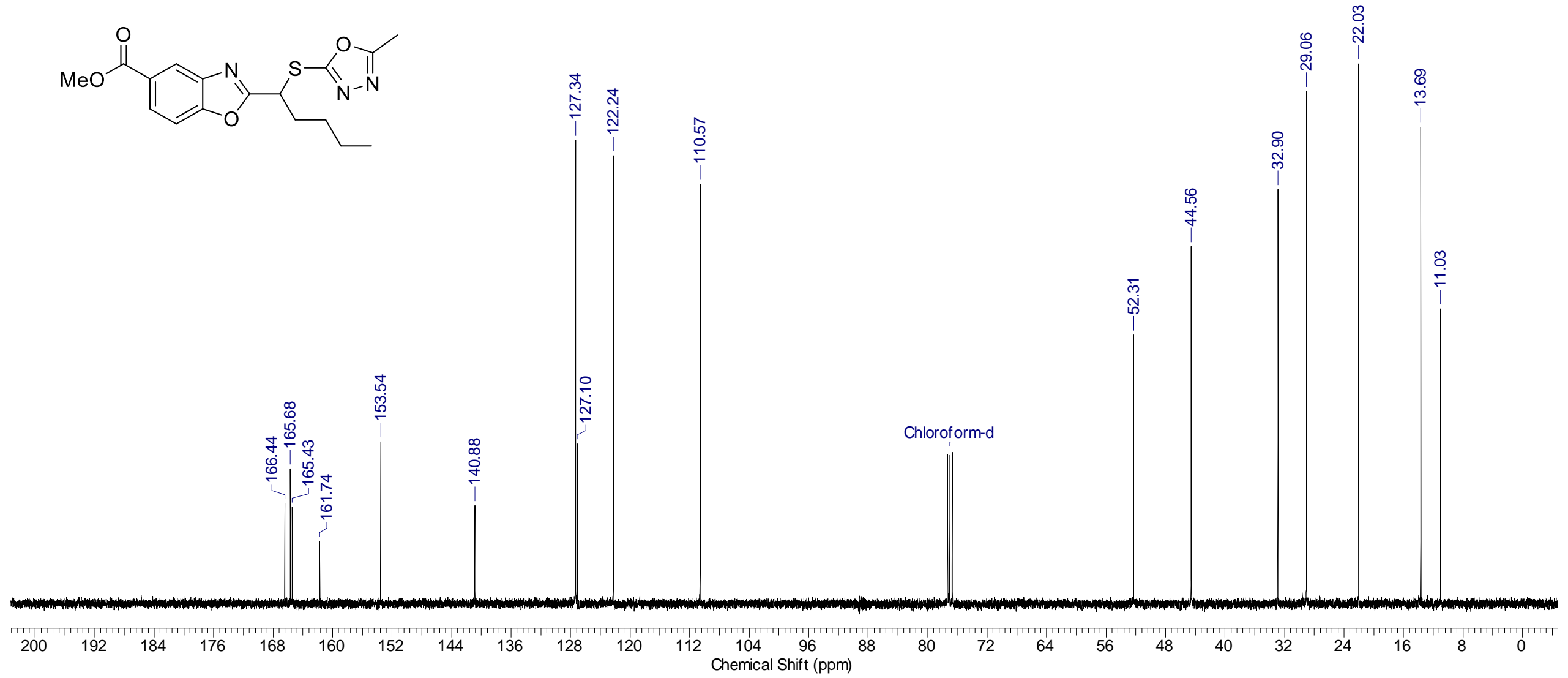




\section{5-Chloro-2-\{1-[(5-methyl-1,3,4-oxadiazol-2-yl)thio]pentyl\}-1,3-benzoxazole (2s)}

${ }^{1} \mathrm{H}$ NMR (400 MHz, $\mathrm{CDCl}_{3}$ )
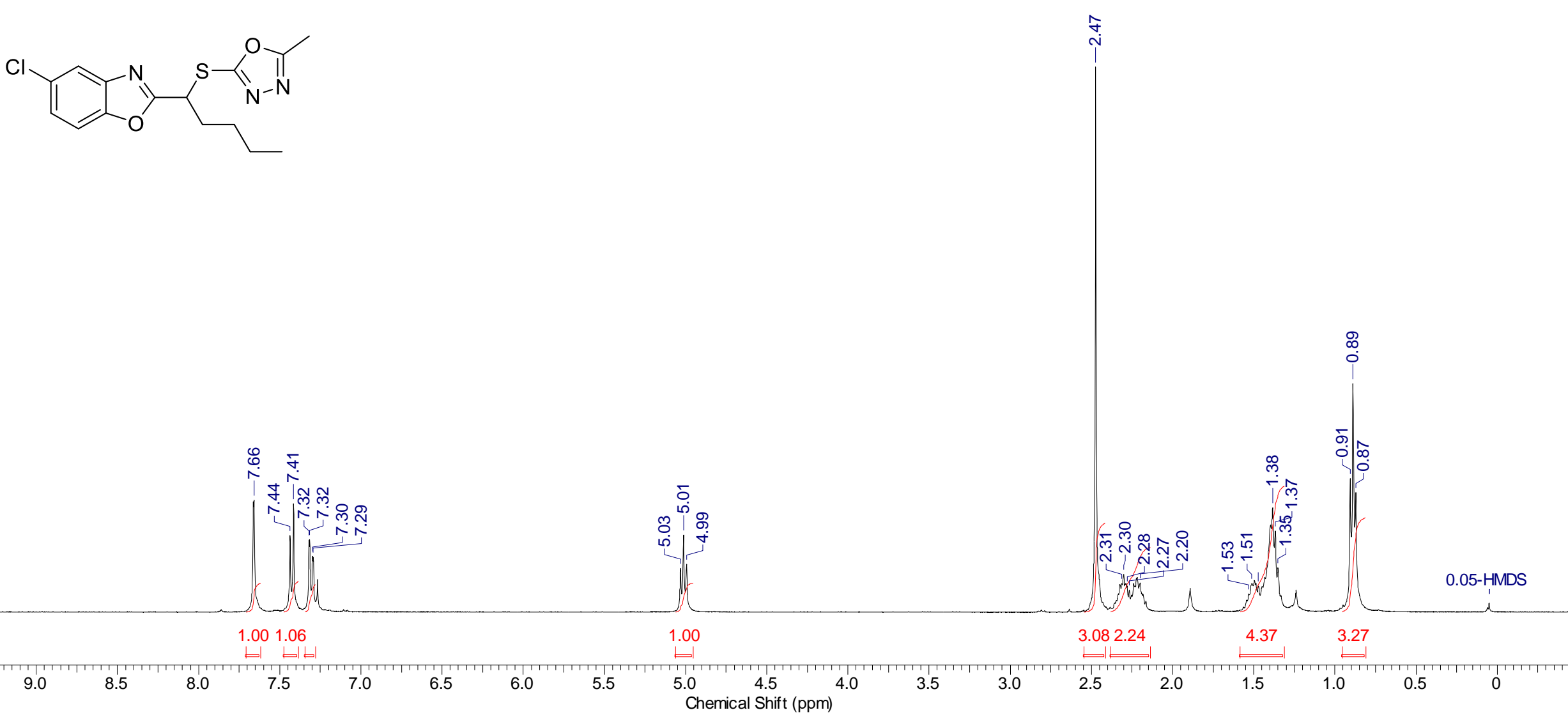
5-Chloro-2-\{1-[(5-methyl-1,3,4-oxadiazol-2-yl)thio]pentyl\}-1,3-benzoxazole (2s)

${ }^{13} \mathrm{C}$ NMR (100.6 MHz, $\left.\mathrm{CDCl}_{3}\right)$

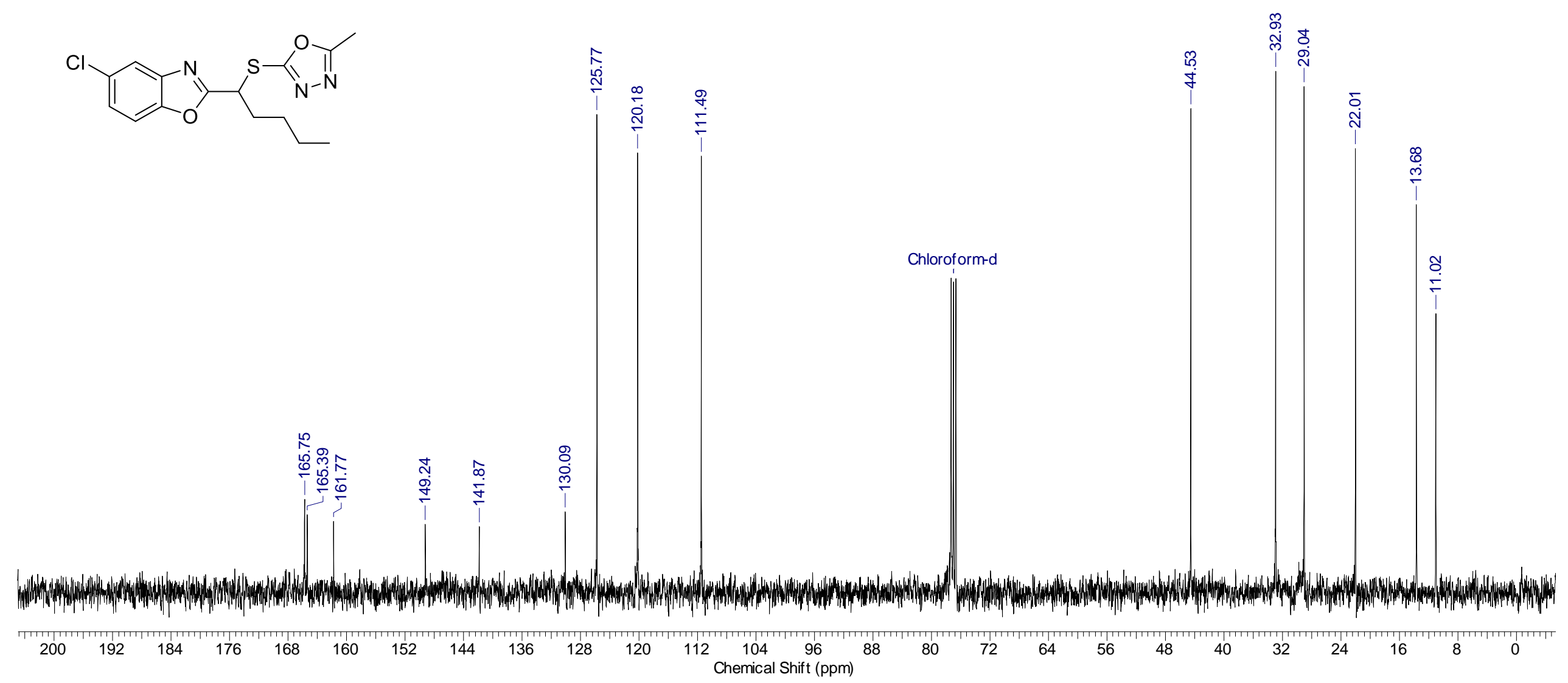




\section{5-Iodo-2-\{1-[(5-methyl-1,3,4-oxadiazol-2-yl)thio]pentyl\}-1,3-benzoxazole (2t)}

${ }^{1} \mathrm{H}$ NMR (600 MHz, $\mathrm{CDCl}_{3}$ )
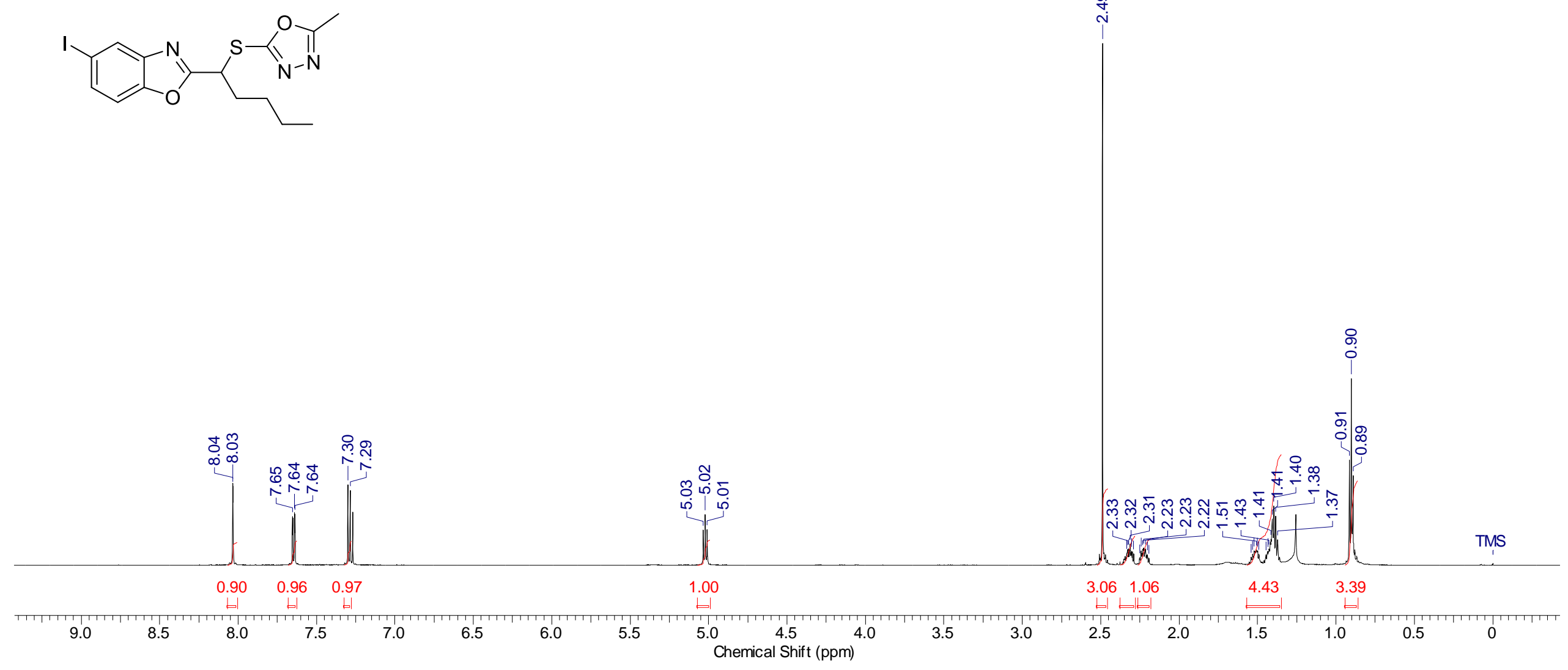
5-Iodo-2-\{1-[(5-methyl-1,3,4-oxadiazol-2-yl)thio]pentyl\}-1,3-benzoxazole (2t)

${ }^{13} \mathrm{C}$ NMR (151 MHz, $\mathrm{CDCl}_{3}$ )

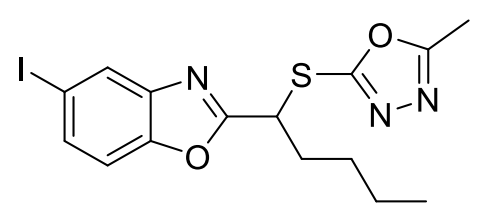

Chloroform-d

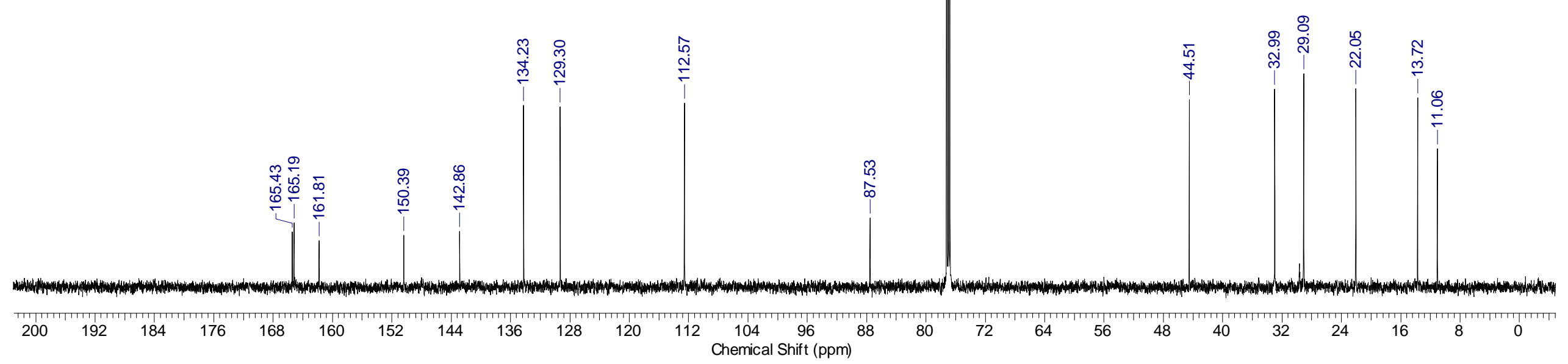


(7-Methoxy-2-\{1-[(5-methyl-1,3,4-oxadiazol-2-yl)thio]pentyl\}-1,3-benzoxazol-5-yl)methanol (2u)

${ }^{1} \mathrm{H}$ NMR (400 MHz, $\left.\mathrm{CDCl}_{3}\right)$

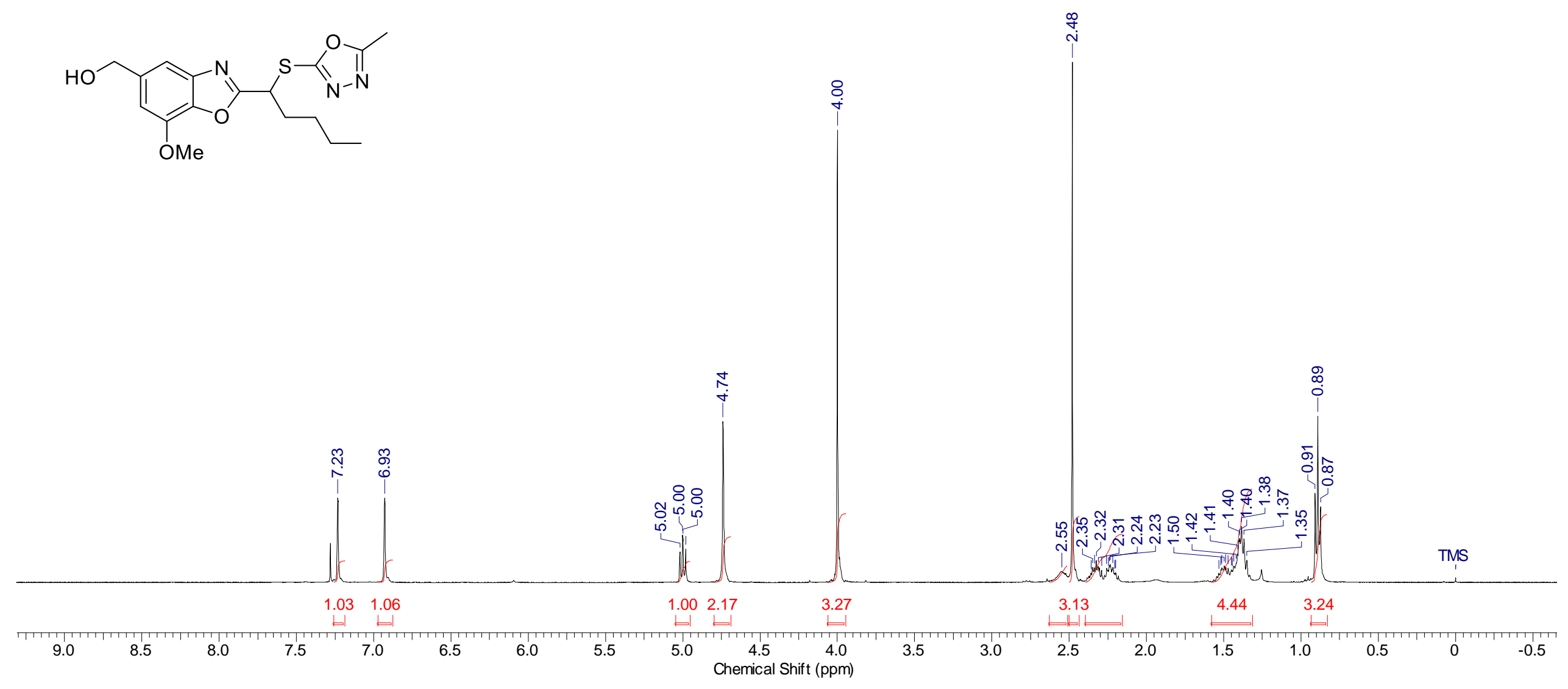


(7-Methoxy-2-\{1-[(5-methyl-1,3,4-oxadiazol-2-yl)thio]pentyl\}-1,3-benzoxazol-5-yl)methanol (2u)

${ }^{13} \mathrm{C}$ NMR (100.6 MHz, $\left.\mathrm{CDCl}_{3}\right)$

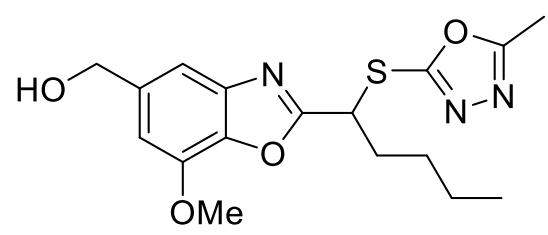

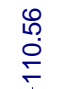

苍

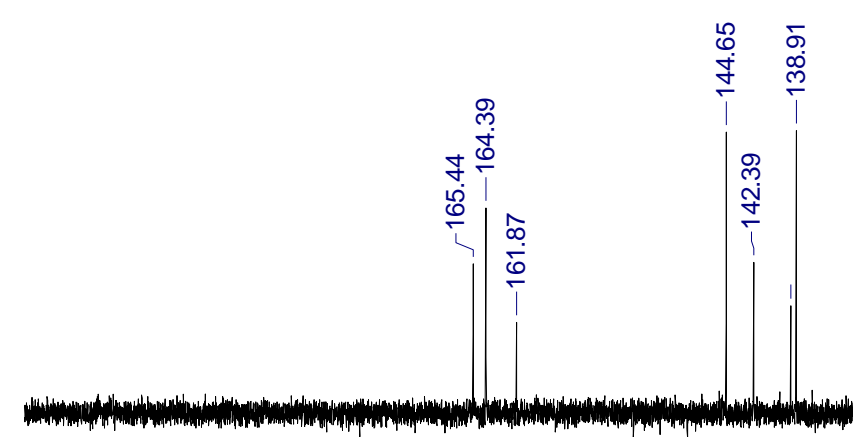

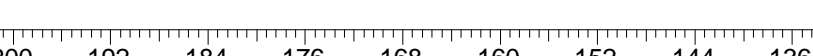
$128 \quad 120$ 1121049 $\begin{array}{ccc}104 & 96 & 88 \\ \text { Chemical Shift (ppm) }\end{array}$ 
7-Bromo-5-methyl-2-\{1-[(5-methyl-1,3,4-oxadiazol-2-yl)thio]pentyl\}-1,3-benzoxazole (2v)

${ }^{1} \mathrm{H}$ NMR (400 MHz, $\left.\mathrm{CDCl}_{3}\right)$

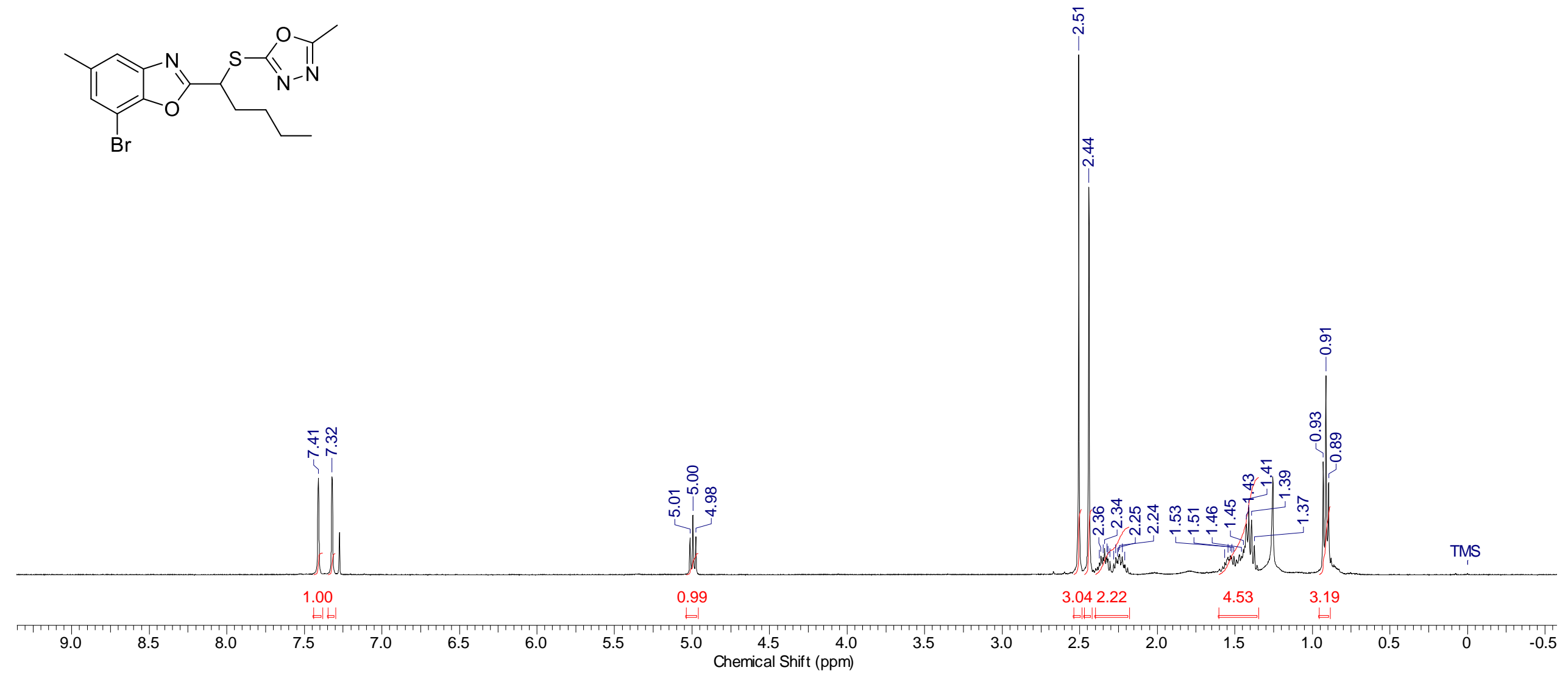


7-Bromo-5-methyl-2-\{1-[(5-methyl-1,3,4-oxadiazol-2-yl)thio]pentyl\}-1,3-benzoxazole (2v)

${ }^{13} \mathrm{C}$ NMR (100.6 MHz, $\mathrm{CDCl}_{3}$ )

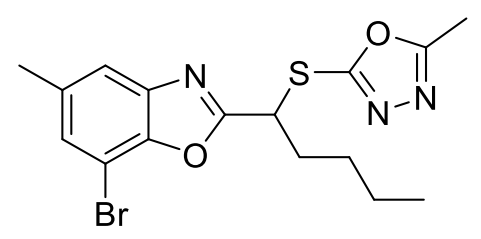

Chloroform-d

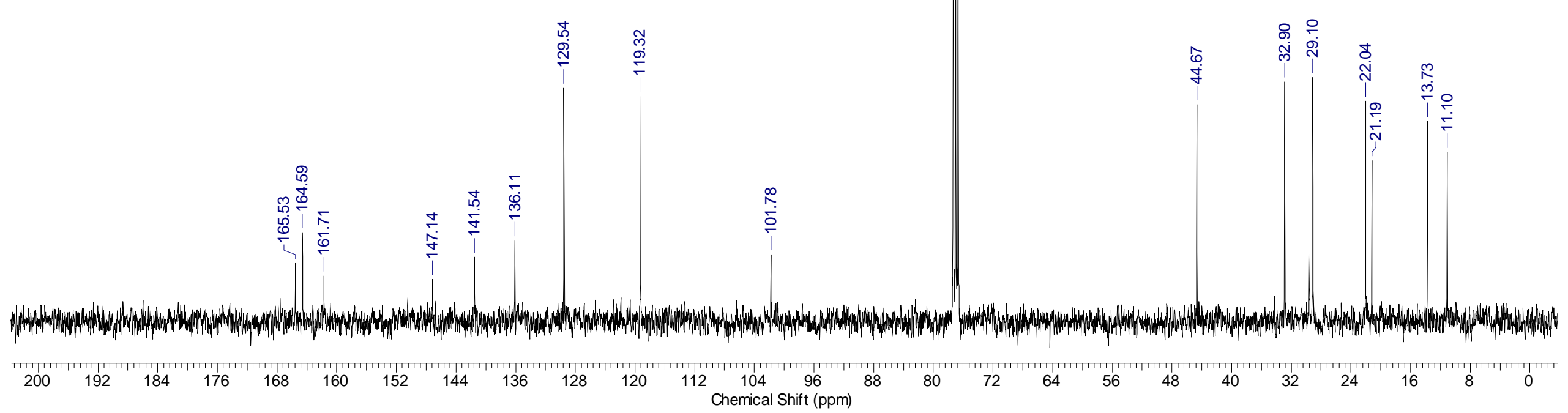


2-\{4-Methyl-1-[(5-methyl-1,3,4-oxadiazol-2-yl)thio]pentyl\}-1,3-benzoxazole (2w)

${ }^{1} \mathrm{H} \mathrm{NMR}\left(600 \mathrm{MHz}, \mathrm{CDCl}_{3}\right)$
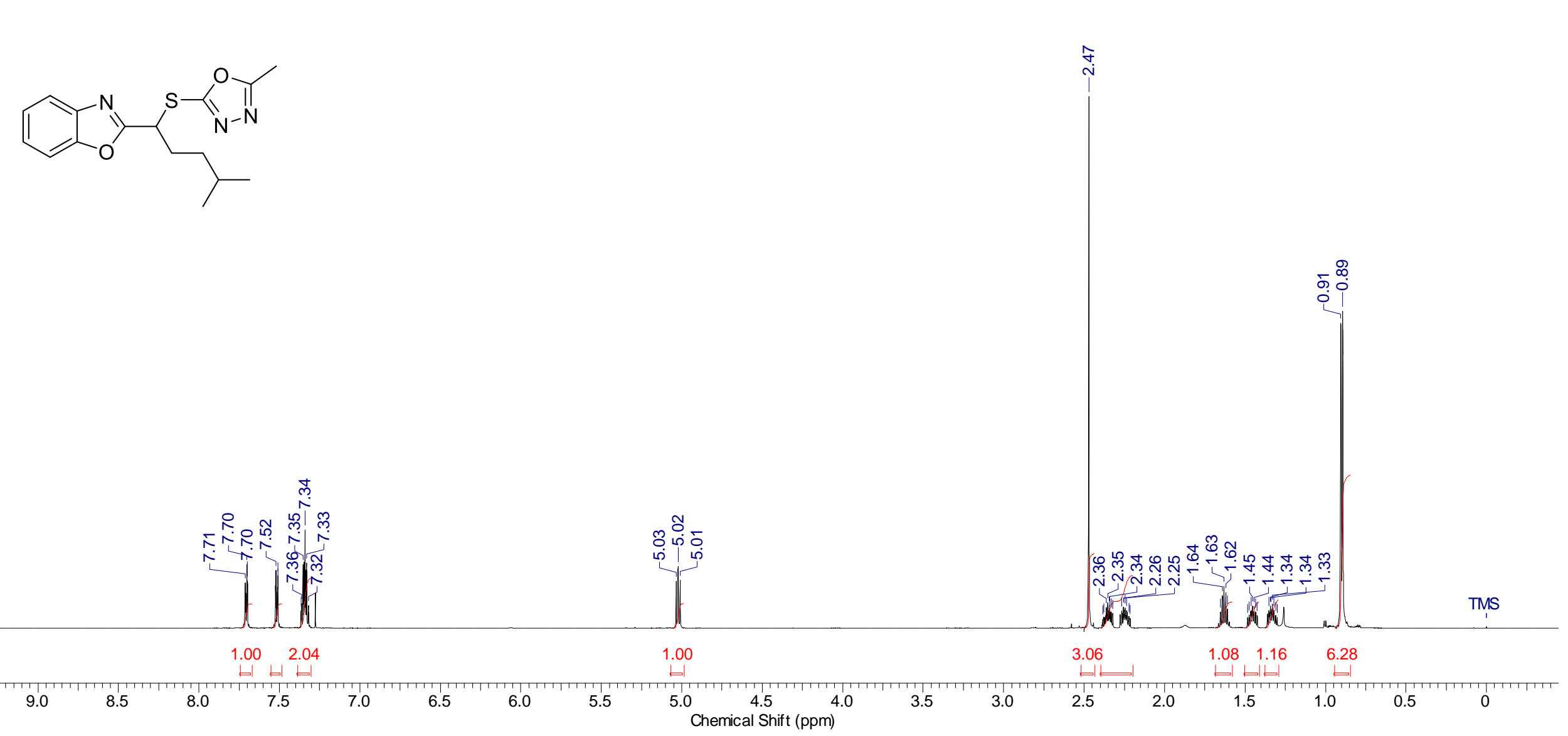
2-\{4-Methyl-1-[(5-methyl-1,3,4-oxadiazol-2-yl)thio]pentyl\}-1,3-benzoxazole (2w)

${ }^{13} \mathrm{C}$ NMR (151 MHz, $\mathrm{CDCl}_{3}$ )
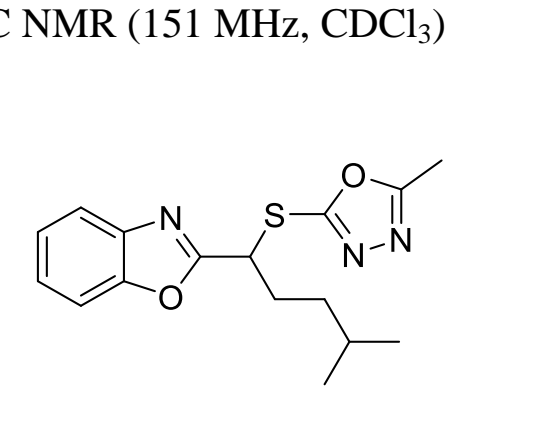


\section{2-\{1-[(5-Methyl-1,3,4-oxadiazol-2-yl)thio]pentyl\}-6-nitro-1,3-benzoxazole (2x)}

${ }^{1} \mathrm{H}$ NMR (400 MHz, $\mathrm{CDCl}_{3}$ )
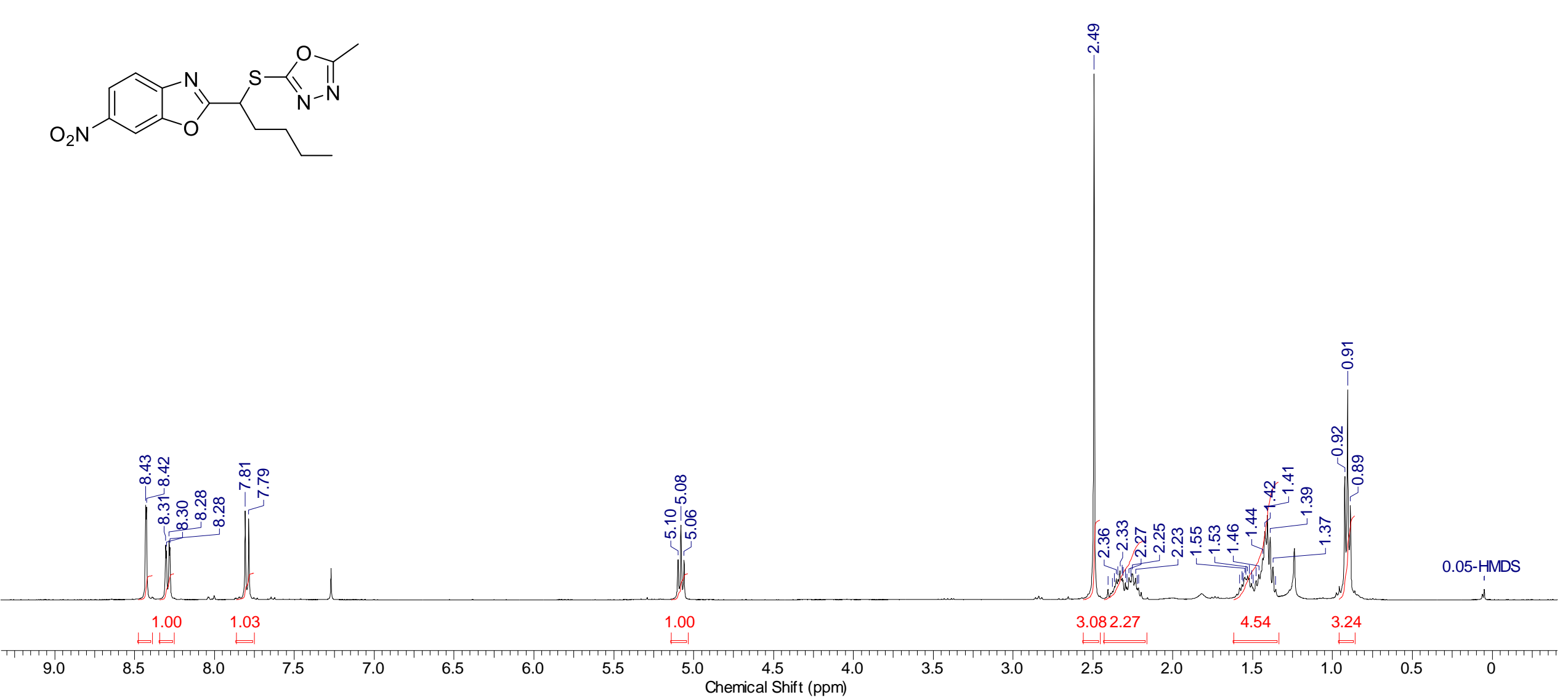


\section{2-\{1-[(5-Methyl-1,3,4-oxadiazol-2-yl)thio]pentyl\}-6-nitro-1,3-benzoxazole (2x)}

${ }^{13} \mathrm{C}$ NMR (100.6 MHz, $\left.\mathrm{CDCl}_{3}\right)$
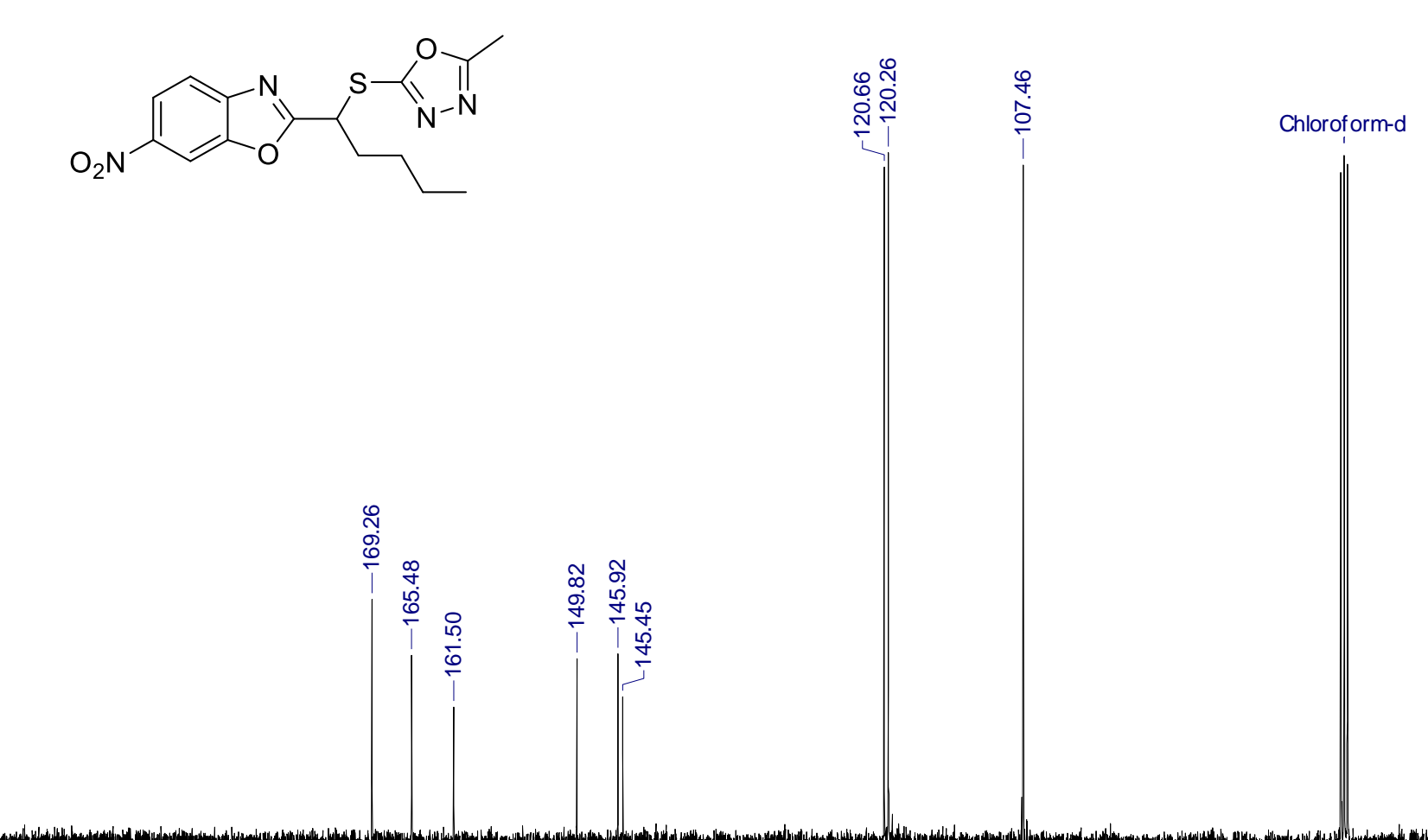

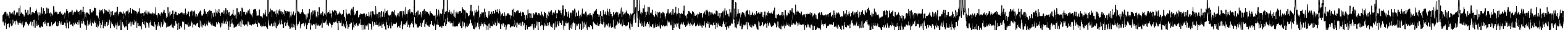

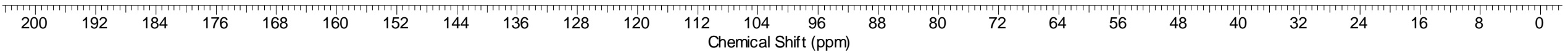




\section{2-\{Cyclohexyl[(5-methyl-1,3,4-oxadiazol-2-yl)thio]methyl\}-1,3-benzoxazole (2y)}

${ }^{1} \mathrm{H}$ NMR (600 MHz, $\mathrm{CDCl}_{3}$ )

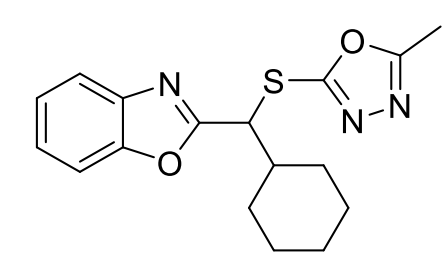

$$
\text { (1) }
$$

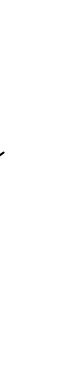


2-\{Cyclohexyl[(5-methyl-1,3,4-oxadiazol-2-yl)thio]methyl\}-1,3-benzoxazole (2y)

${ }^{13} \mathrm{C}$ NMR (151 MHz, $\left.\mathrm{CDCl}_{3}\right)$

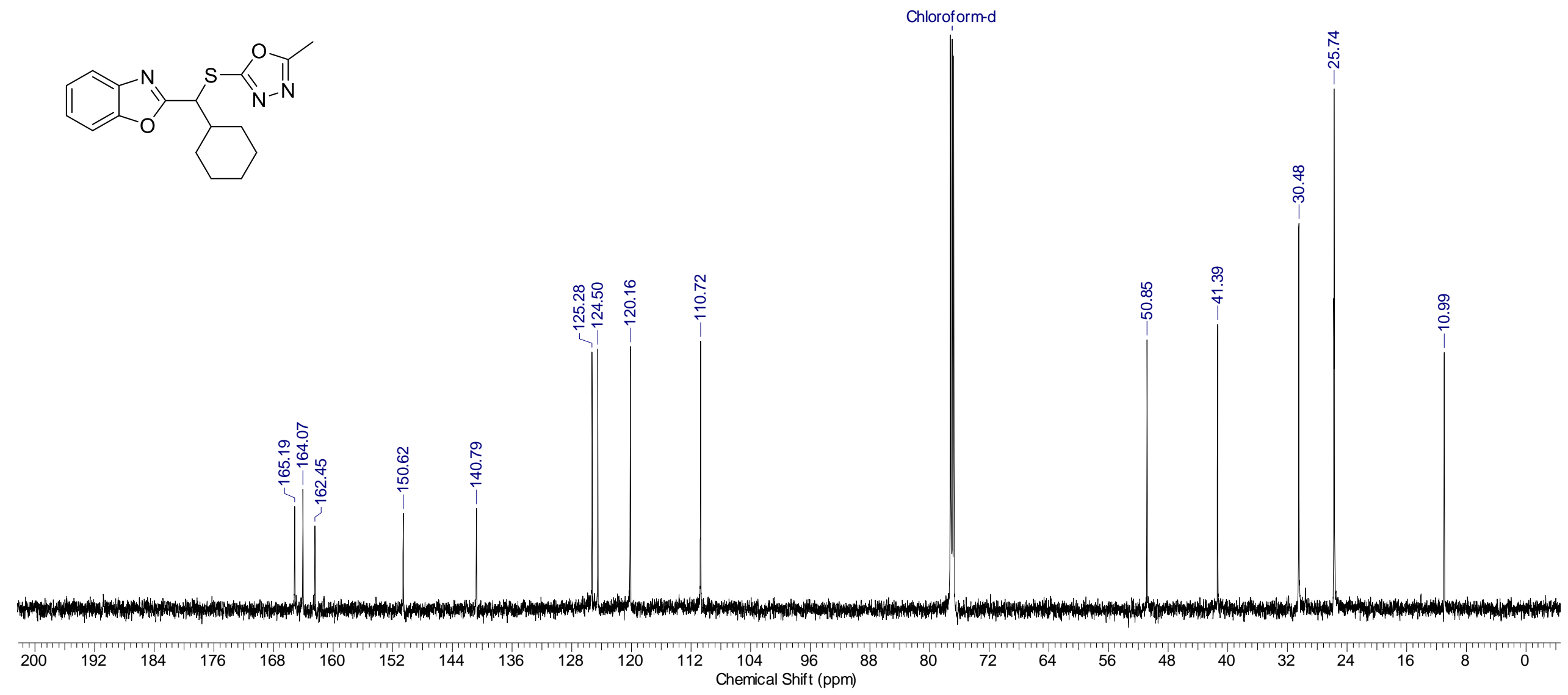




\section{2-\{2-Cyclohexyl-1-[(5-methyl-1,3,4-oxadiazol-2-yl)thio]ethyl\}-1,3-benzoxazole (2z)}

${ }^{1} \mathrm{H}$ NMR (600 MHz, $\mathrm{CDCl}_{3}$ )
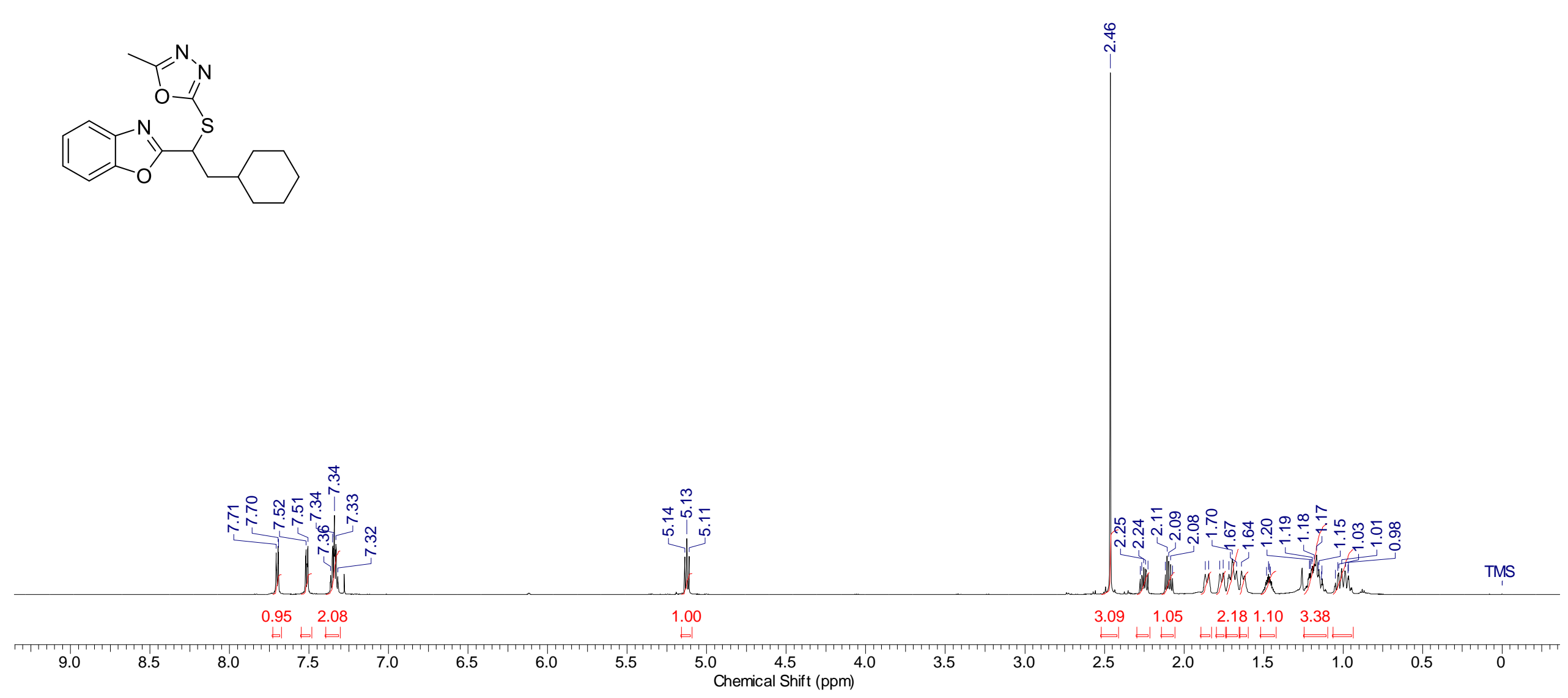
2-\{2-Cyclohexyl-1-[(5-methyl-1,3,4-oxadiazol-2-yl)thio]ethyl\}-1,3-benzoxazole (2z)

${ }^{13} \mathrm{C}$ NMR (151 MHz, $\mathrm{CDCl}_{3}$ )
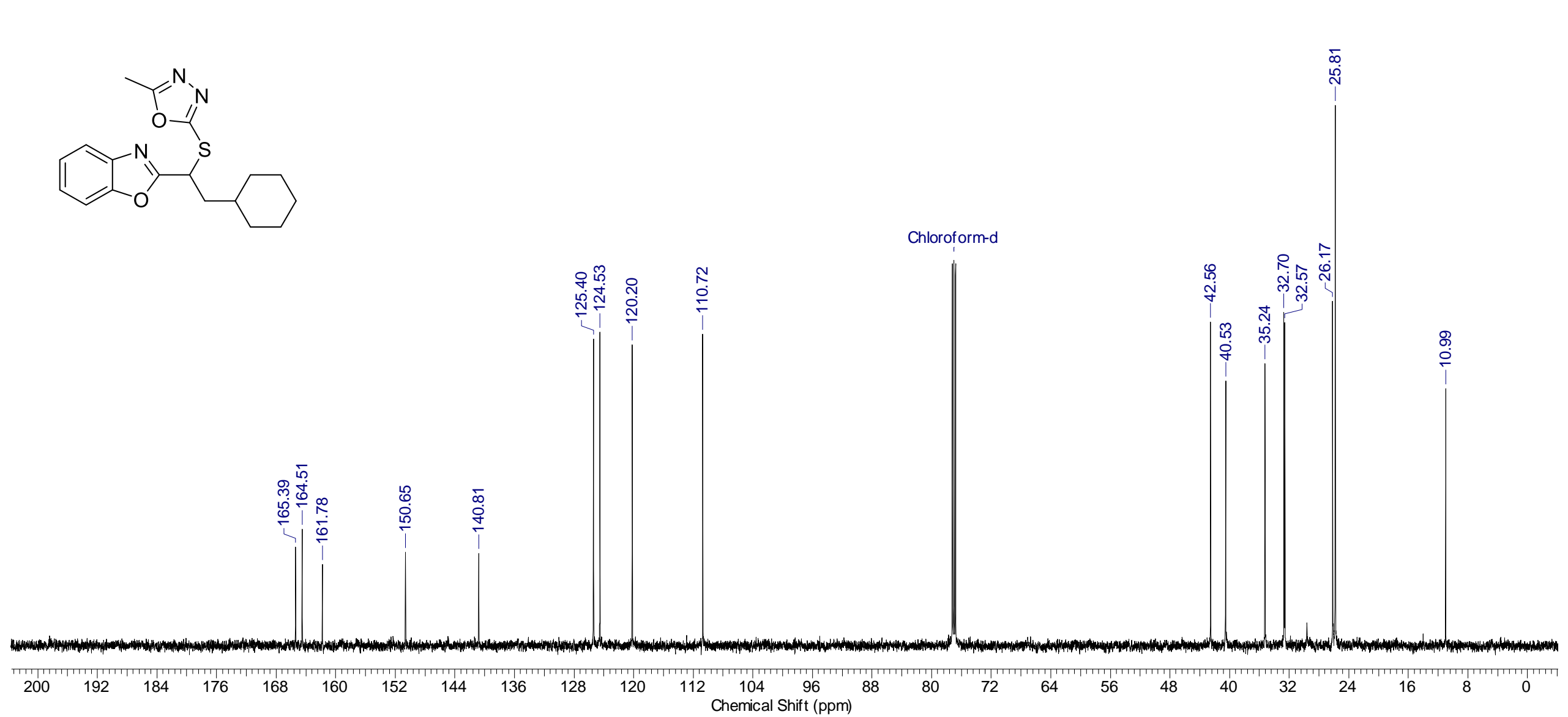


\section{2-\{1-[(5-Methyl-1,3,4-oxadiazol-2-yl)thio]-2-phenylethyl\}-1,3-benzoxazole (2aa)}

${ }^{1} \mathrm{H}$ NMR (400 MHz, $\mathrm{CDCl}_{3}$ )

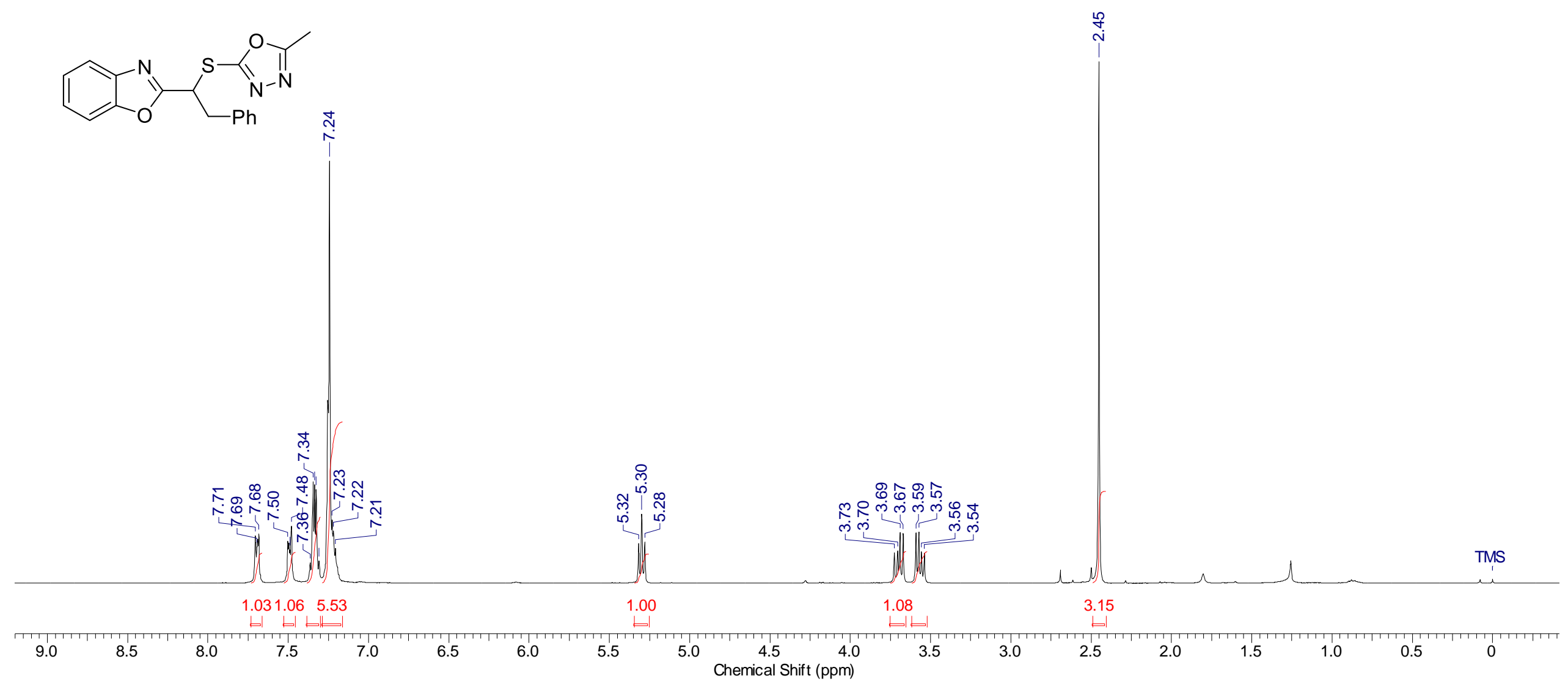


2-\{1-[(5-Methyl-1,3,4-oxadiazol-2-yl)thio]-2-phenylethyl\}-1,3-benzoxazole (2aa)

${ }^{13} \mathrm{C}$ NMR (100.6 MHz, $\left.\mathrm{CDCl}_{3}\right)$
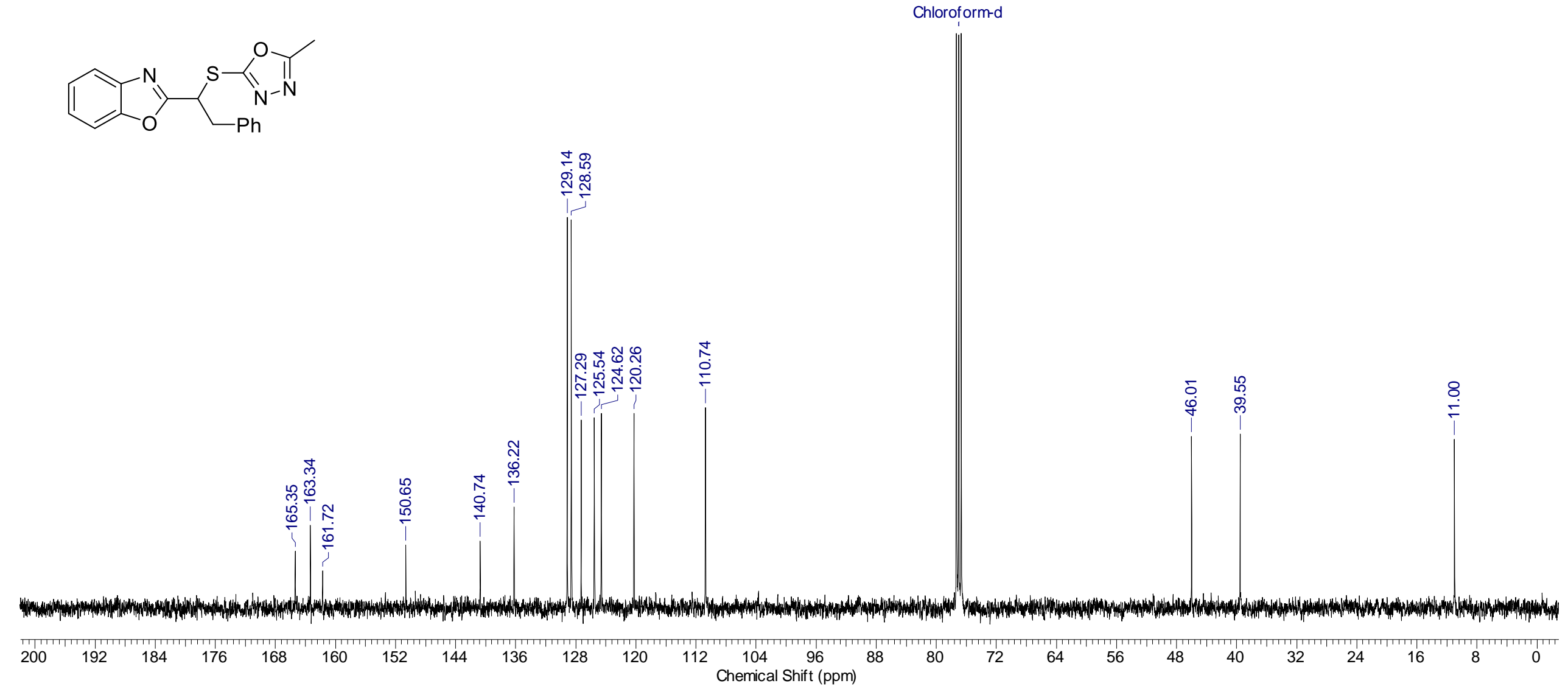


\section{3-(1,3-Benzoxazol-2-yl)-3-[(5-methyl-1,3,4-oxadiazol-2-yl)thio]propan-1-ol (2ac)}

${ }^{1} \mathrm{H}$ NMR (400 MHz, $\mathrm{CDCl}_{3}$ )
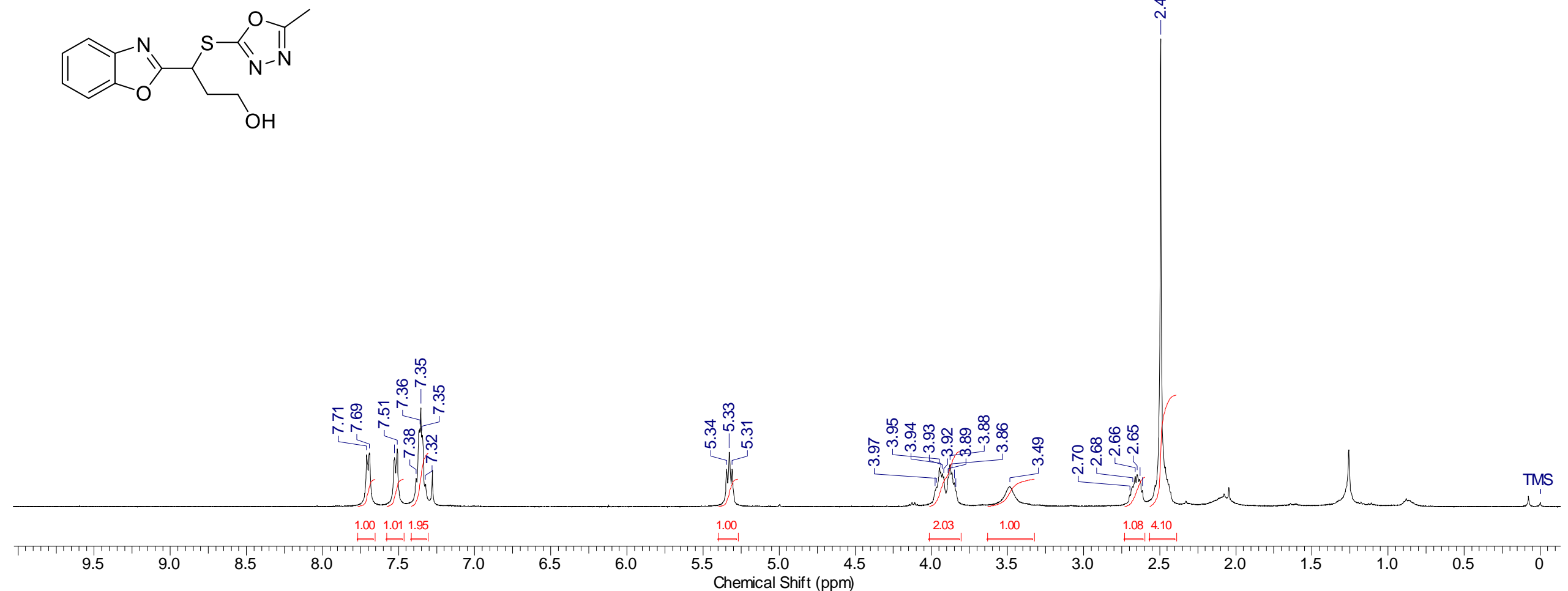
3-(1,3-Benzoxazol-2-yl)-3-[(5-methyl-1,3,4-oxadiazol-2-yl)thio]propan-1-ol (2ac)

${ }^{13} \mathrm{C}$ NMR (100.6 MHz, $\left.\mathrm{CDCl}_{3}\right)$
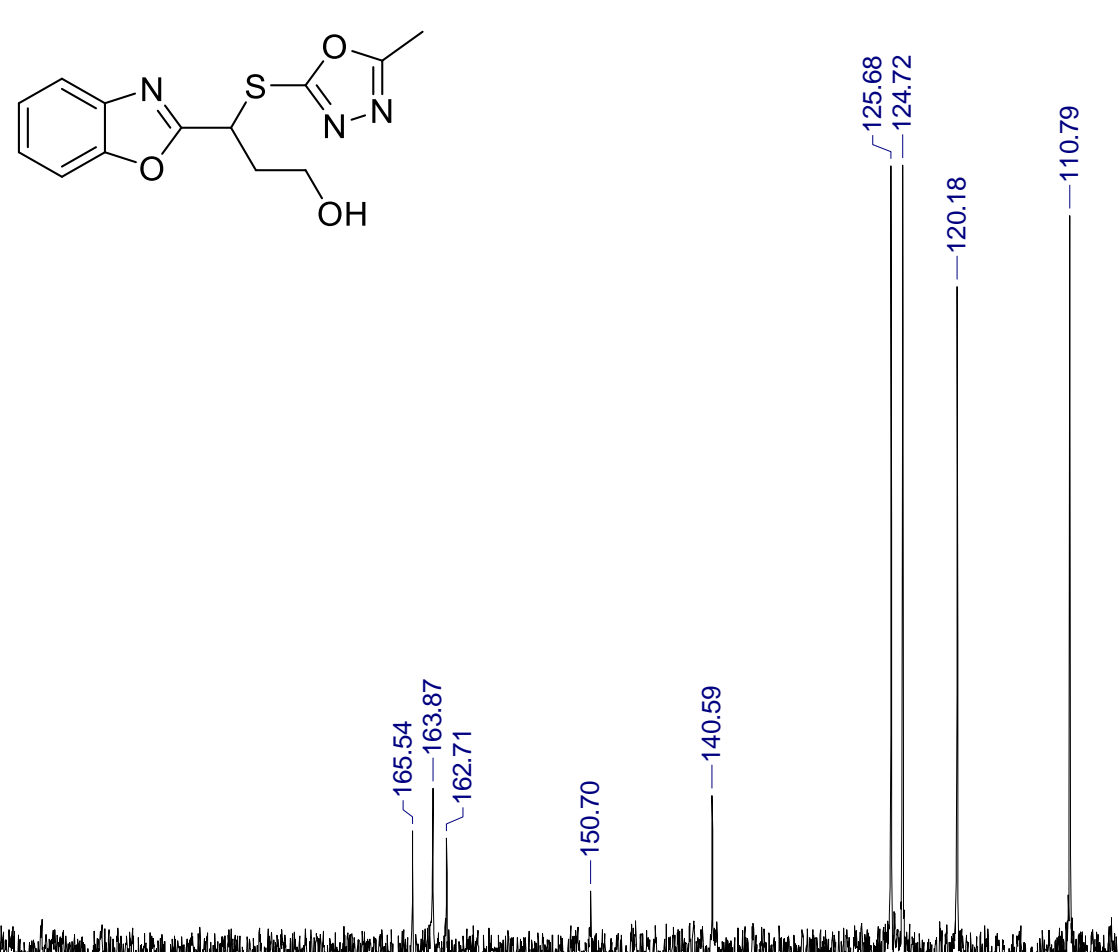

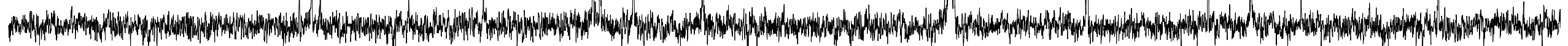

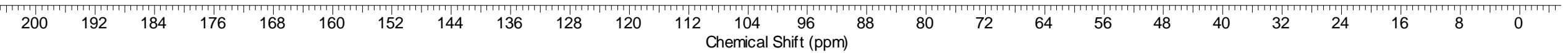




\section{$N$-\{2-(1,3-Benzoxazol-2-yl)-2-[(5-methyl-1,3,4-oxadiazol-2-yl)thio]ethyl\}acetamide (2ad)}

${ }^{1} \mathrm{H}$ NMR (600 MHz, $\mathrm{CDCl}_{3}$ )

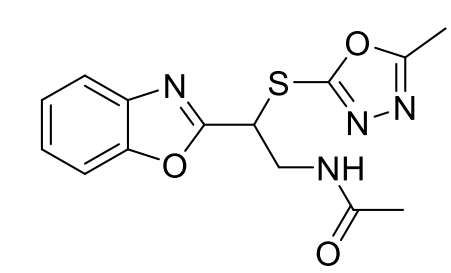

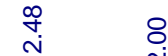

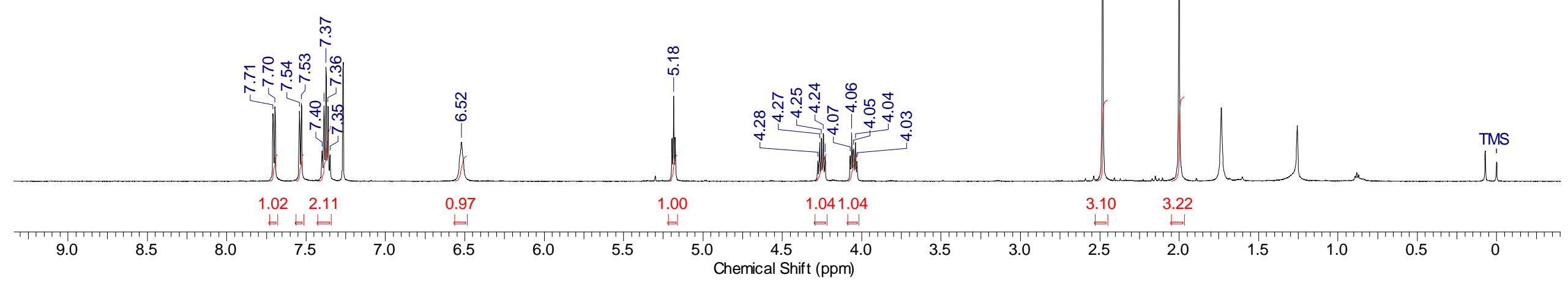


$N$-\{2-(1,3-Benzoxazol-2-yl)-2-[(5-methyl-1,3,4-oxadiazol-2-yl)thio]ethyl\}acetamide (2ad)

${ }^{13} \mathrm{C}$ NMR (100.6 MHz, $\left.\mathrm{CDCl}_{3}\right)$

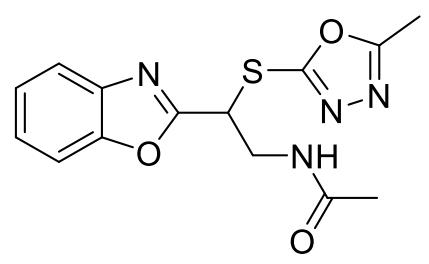

Chloroform-d

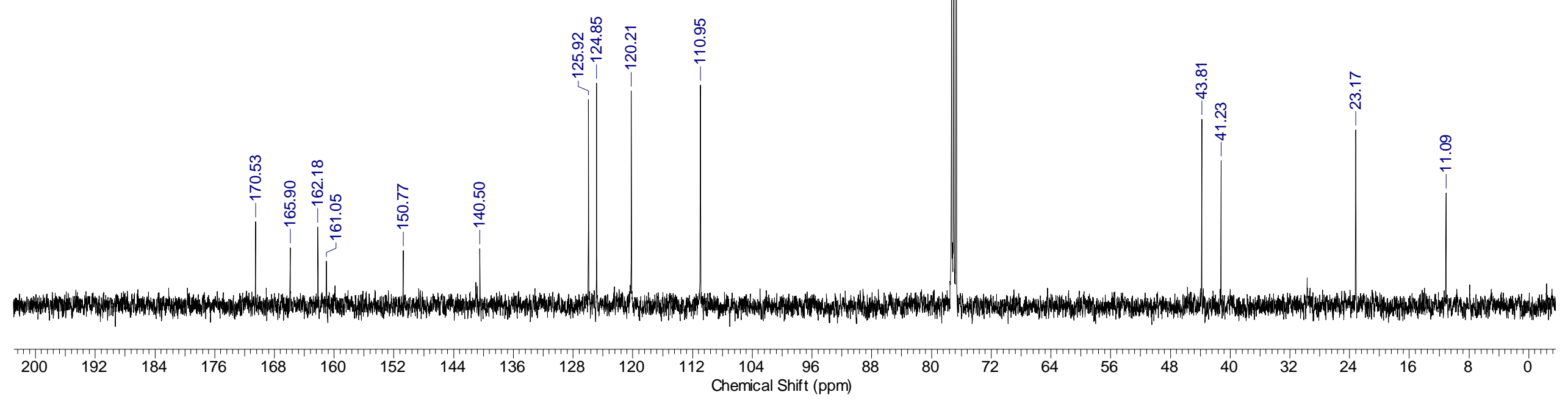


Dimethyl \{2-(1,3-benzoxazol-2-yl)-2-[(5-methyl-1,3,4-oxadiazol-2-yl)thio]ethyl\}(methyl)malonate (2ae)

${ }^{1} \mathrm{H}$ NMR $\left(400 \mathrm{MHz}, \mathrm{CDCl}_{3}\right)$

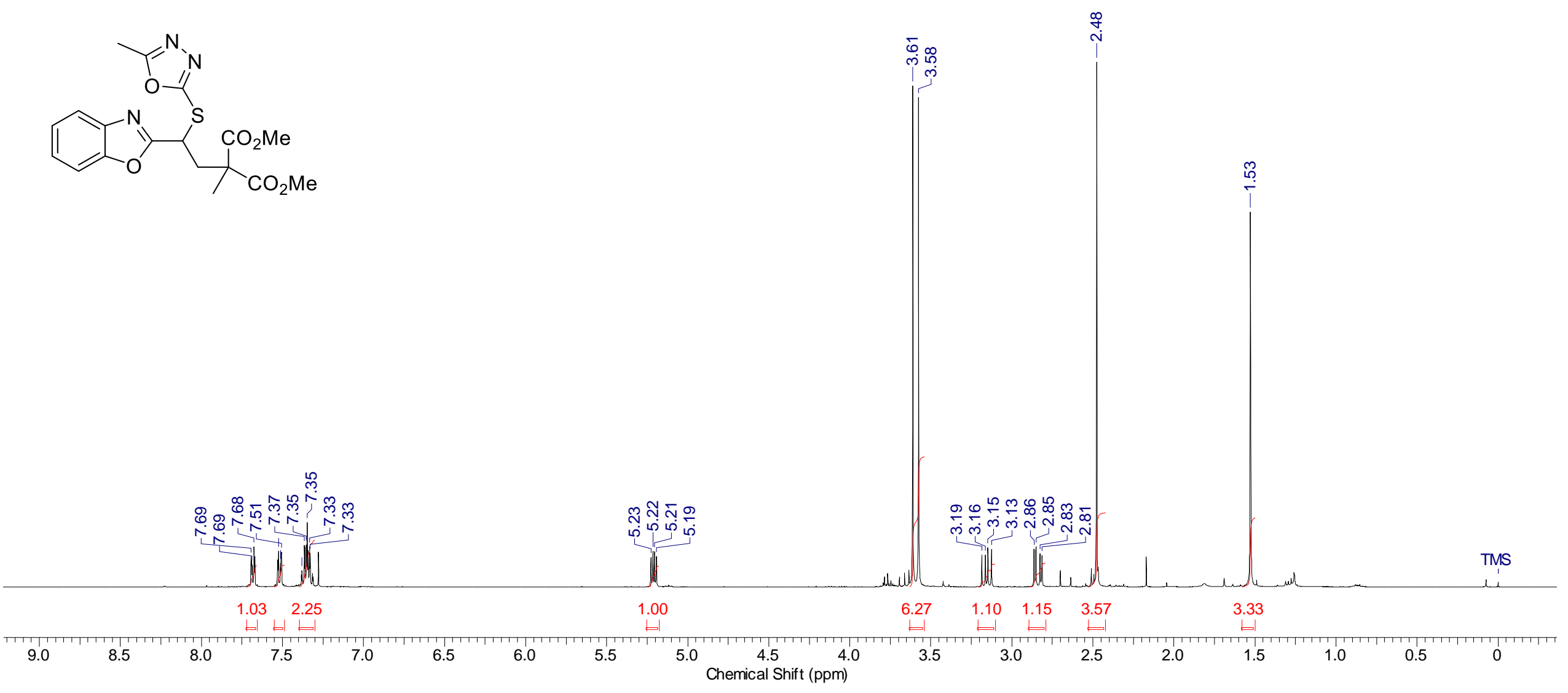


Dimethyl \{2-(1,3-benzoxazol-2-yl)-2-[(5-methyl-1,3,4-oxadiazol-2-yl)thio]ethyl\}(methyl)malonate (2ae)

${ }^{13} \mathrm{C}$ NMR (100.6 MHz, $\left.\mathrm{CDCl}_{3}\right)$

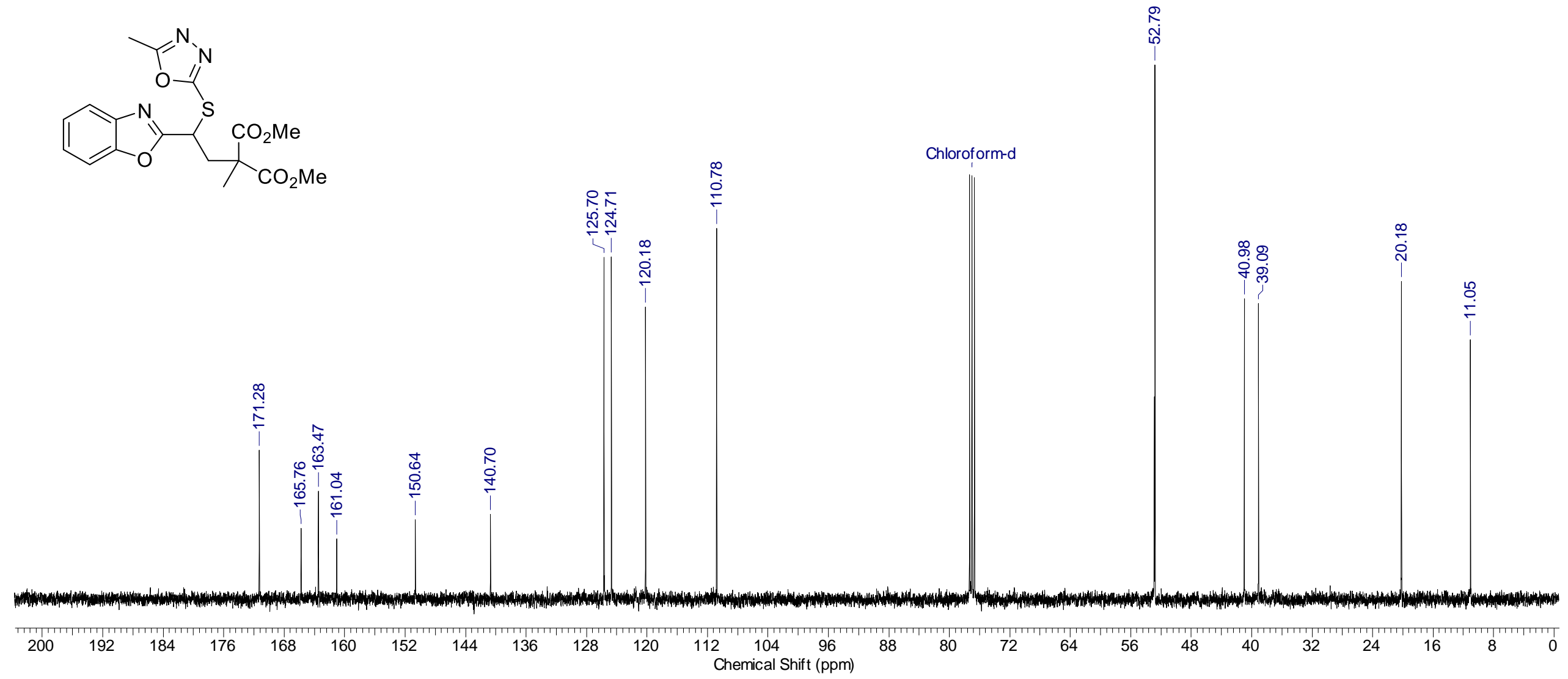




\section{2-(4-Butyl-1H-1,2,3-triazol-1-yl)phenol (5)}

${ }^{1} \mathrm{H}$ NMR (400 MHz, $\mathrm{CDCl}_{3}$ )

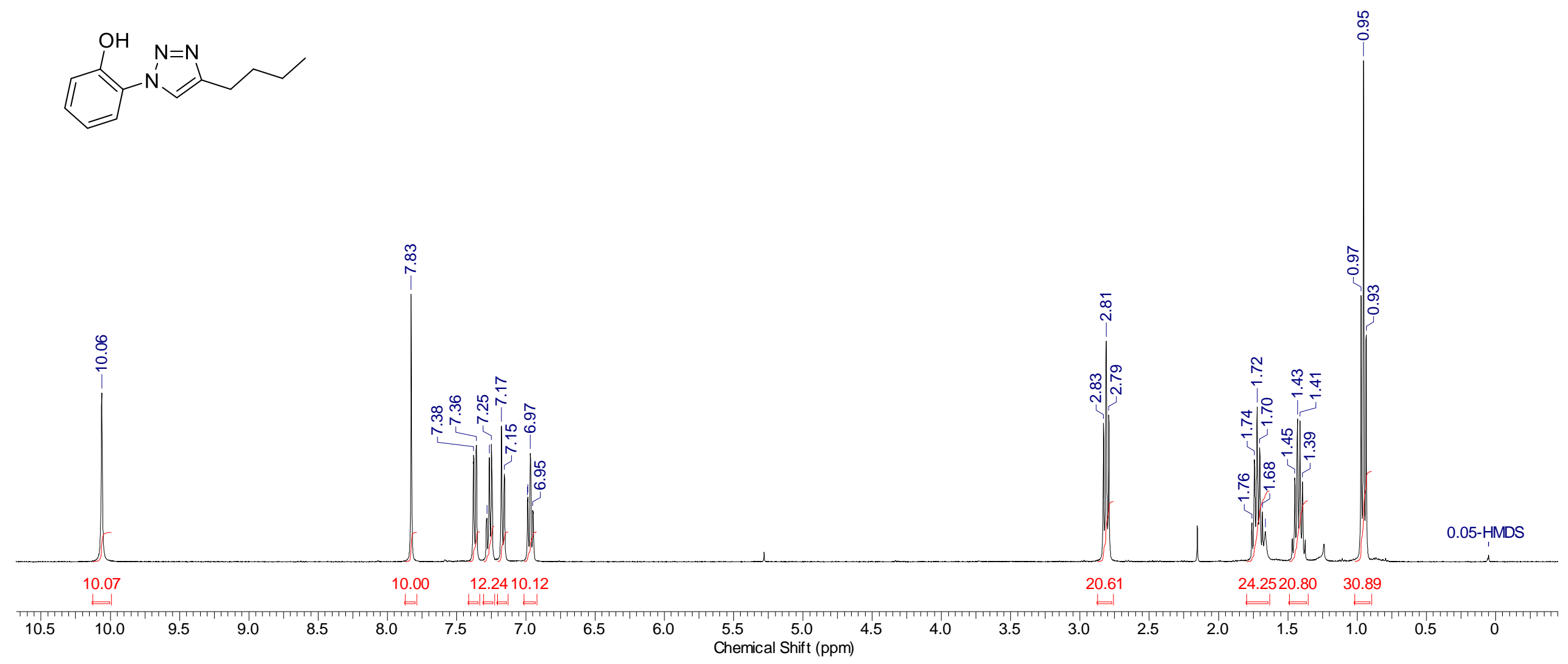


2-(4-Butyl-1H-1,2,3-triazol-1-yl)phenol (5)

${ }^{13} \mathrm{C}$ NMR (100.6 MHz, $\mathrm{CDCl}_{3}$ )

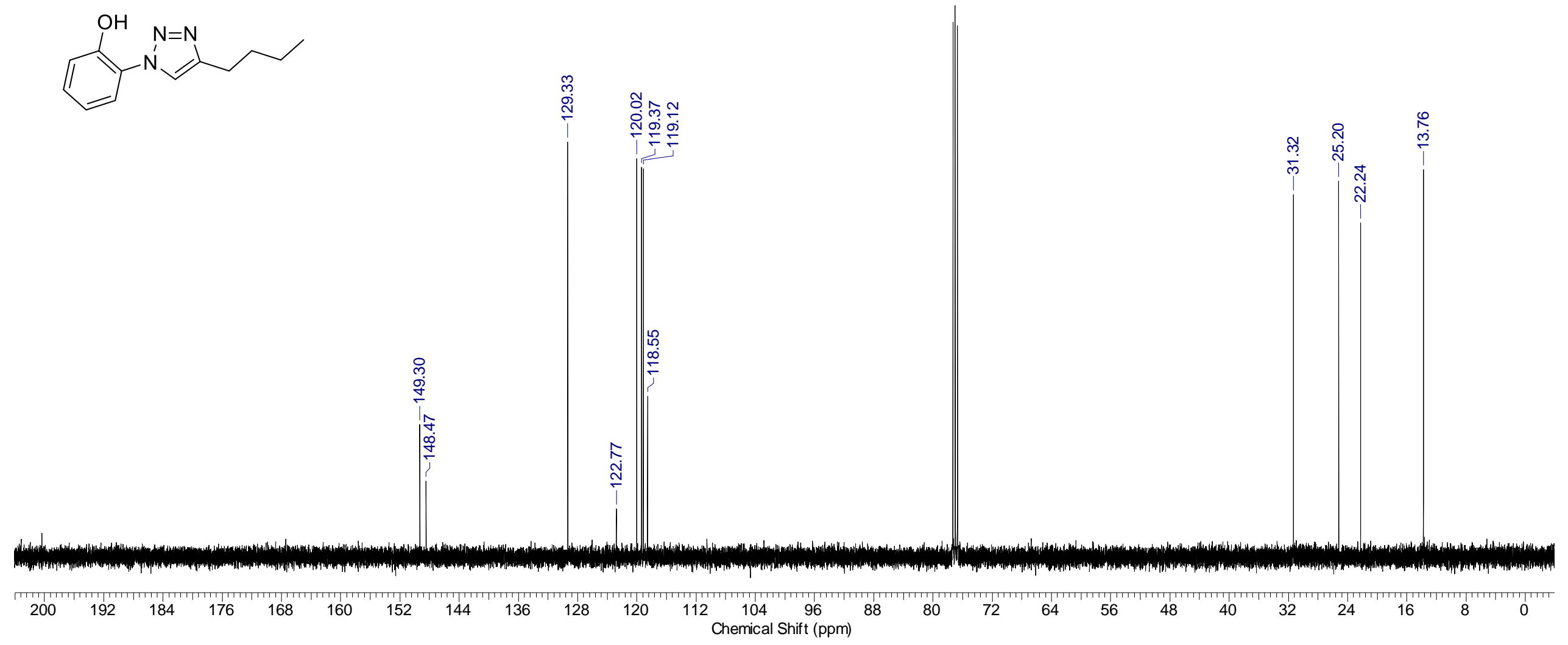


3-\{2-(1,3-Benzoxazol-2-yl)-2-[(5-methyl-1,3,4-oxadiazol-2-yl)thio]ethyl\}-5-methyl-1,3,4-oxadiazole-2(3H)-thione (8a)

${ }^{1} \mathrm{H}$ NMR $\left(400 \mathrm{MHz}, \mathrm{CDCl}_{3}\right)$
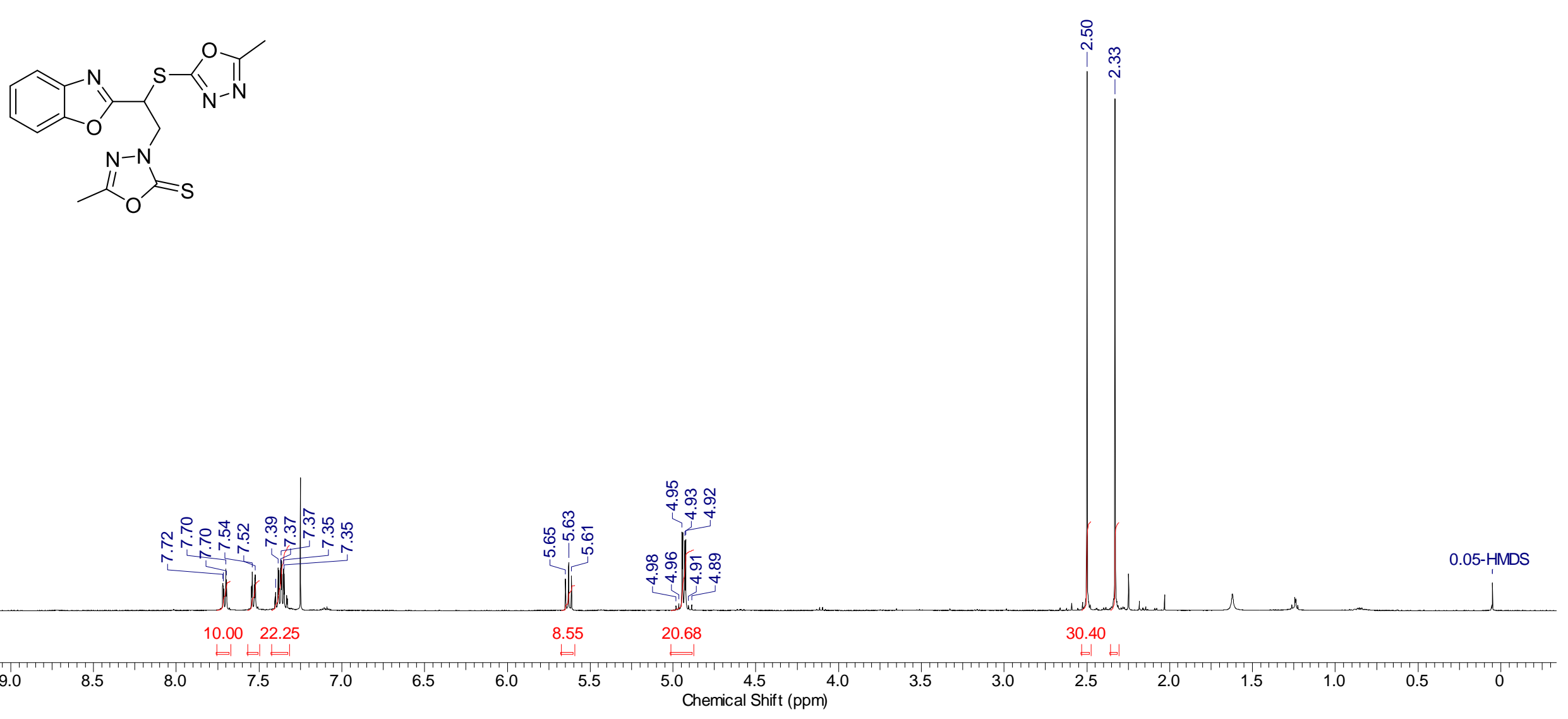
${ }^{13} \mathrm{C}$ NMR (100.6 MHz, $\mathrm{CDCl}_{3}$ )

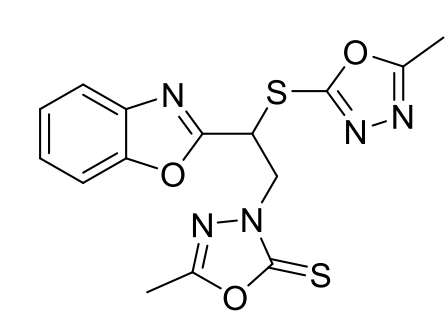

$$
\text { Chloroform-d }
$$

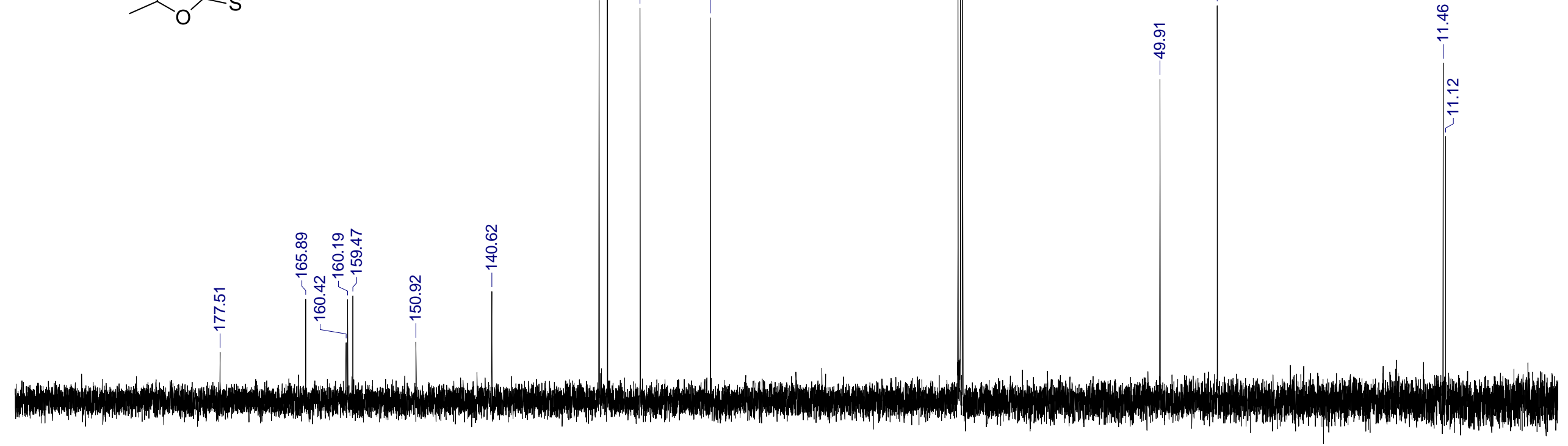

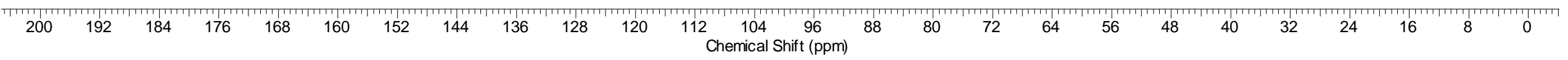




\section{1-\{2-(1,3-Benzoxazol-2-yl)-2-[(1-methyl-1H-imidazol-2-yl)thio]ethyl\}-3-methyl-1,3-dihydro-2H-imidazole-2-thione (8b)}

${ }^{1} \mathrm{H}$ NMR (400 MHz, $\mathrm{CDCl}_{3}$ )

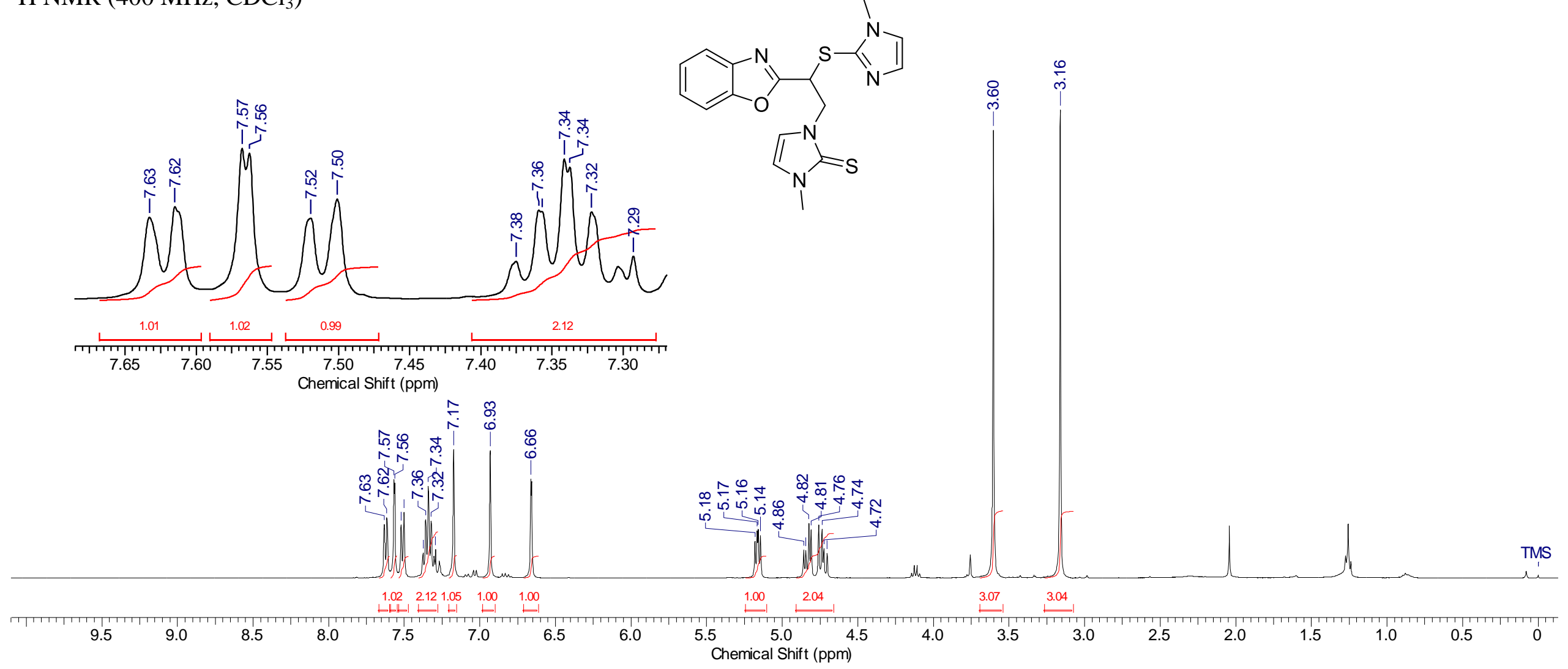


${ }^{13} \mathrm{C}$ NMR (100.6 MHz, $\left.\mathrm{CDCl}_{3}\right)$

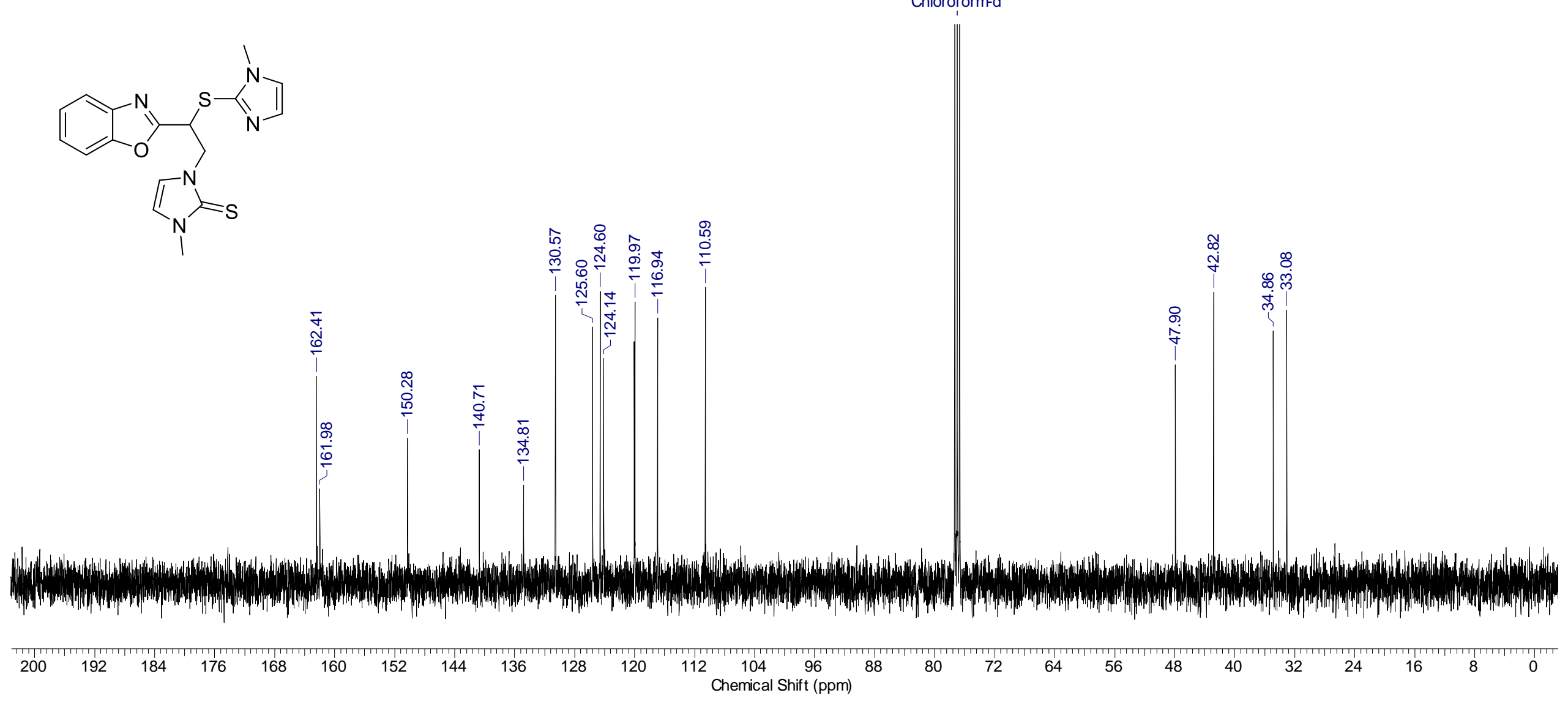




\section{References}

${ }^{1}$ F. Neese. WIREs Comput. Mol. Sci. 2018, 8, e1327.

${ }^{2}$ A. D. Becke. J. Chem. Phys. 1993, 98, 5648-5652.

${ }^{3}$ P. J. Stephens, F. J. Devlin, C. F. Chabalowski, M. J. Frisch. J. Phys. Chem. 1994, 98, $11623-11627$.

${ }^{4}$ F. Weigend, R. Ahlrichs. Phys. Chem. Chem. Phys. 2005, 7, 3297-3305.

${ }^{5}$ J. Zheng, X. Xu, D. G. Truhlar. Theor. Chem. Acc. 2011, 128, 295-305.

${ }^{6}$ K. A. Peterson, D. Figgen, E. Goll, H. Stoll, M. Dolg. J. Chem. Phys. 2003, 119, 11113-11123.

${ }^{7}$ A. V. Marenich, C. J. Cramer, D. G. Truhlar. J. Phys. Chem. B 2009, 113, 6378-6396.

${ }^{8}$ F. Neese, F. Wennmohs, A. Hansen, U. Becker. Chem. Phys. 2009, 356, 98-109.

${ }^{9}$ S. Grimme. Chem. Eur. J. 2012, 18, 9955-9964.

${ }^{10}$ CYLview, 1.0b; C. Y. Legault, Université de Sherbrooke, 2009, http://www.cylview.org. 